
3D FACE RECOGNITION WITH LOCAL SHAPE DESCRIPTORS

A THESIS SUBMITTED TO

THE GRADUATE SCHOOL OF NATURAL AND APPLIED SCIENCES

$\mathrm{OF}$

MIDDLE EAST TECHNICAL UNIVERSITY

BY

TOLGA İNAN

IN PARTIAL FULFILLMENT OF THE REQUIREMENTS

FOR

THE DEGREE OF DOCTOR OF PHILOSOPHY

IN

ELECTRICAL AND ELECTRONICS ENGINEERING

SEPTEMBER 2011 
Approval of the thesis:

\section{D FACE RECOGNITION WITH LOCAL SHAPE DESCRIPTORS}

submitted by TOLGA INAN in partial fulfillment of the requirements for the degree of Doctor of Philosophy in Electrical and Electronics Engineering Department, Middle East Technical University by,

Prof. Dr. Canan Özgen

Dean, Graduate School of Natural and Applied Sciences

Prof. Dr. İsmet Erkmen

Head of Department, Electrical and Electronics Engineering

Prof. Dr. Uğur Halıcı

Supervisor, Electrical and Electronics Eng. Dept., METU

\section{Examining Committee Members:}

Prof. Dr. Kemal Leblebicioğlu

Electrical and Electronics Engineering, METU

Prof. Dr. Uğur Halıcı

Electrical and Electronics Engineering, METU

Asst.Prof.Dr. Didem Gökçay

Informatics Institute,METU

Asst.Prof.Dr. İlkay Ulusoy

Electrical and Electronics Engineering, METU

Asst.Prof. Dr. Pınar Duygulu Şahin

Computer Engineering, Bilkent University

Date: 
I hereby declare that all information in this document has been obtained and presented in accordance with academic rules and ethical conduct. I also declare that, as required by these rules and conduct, I have fully cited and referenced all material and results that are not original to this work.

Name, Last Name: TOLGA İNAN

Signature 


\begin{abstract}
3D FACE RECOGNITION WITH LOCAL SHAPE DESCRIPTORS

İnan, Tolga

Ph.D., Department of Electrical and Electronics Engineering

Supervisor : Prof. Dr. Uğur Halıcı
\end{abstract}

September 2011, 112 pages

This thesis represents two approaches for three dimensional face recognition. In the first approach, a generic face model is fitted to human face. Local shape descriptors are located on the nodes of generic model mesh. Discriminative local shape descriptors on the nodes are selected and fed as input into the face recognition system. In the second approach, local shape descriptors which are uniformly distributed across the face are calculated. Among the calculated shape descriptors that are discriminative for recognition process are selected and used for three dimensional face recognition.

Both approaches are tested with widely accepted FRGCv2.0 database and experiment protocol. Reported results are better than the state-of-the-art systems. Recognition performances for neutral and non-neutral faces are also reported.

Keywords: 3D Face Recognition, Shape Maps, SIFT,SURF 


\title{
ÖZ
}

\section{YEREL ŞEKİL BETIMLEYİCILER İLE ÜÇ BOYUTLU YÜZ TANIMA}

\author{
İnan, Tolga \\ Doktora, Elektrik ve Elektronik Mühendisliği Bölümü \\ Tez Yöneticisi : Prof. Dr. Uğur Halıcı
}

Eylül 2011, 112 sayfa

Bu tezde, üç boyutlu yüz tanıma için iki ayrı yaklaşım sunulmuştur. Birinci yaklaşımda, jenerik bir yüz modeli insan yüzüne oturtulmuştur. Yerel şekil betimleyicileri, jenerik yüz modelinin düğüm noktalarına yerleştirilmiştir. Düğüm noktalarındaki ayırıcı yerel şekil betimleyiciler seçilmiş ve yüz tanıma sistemine girdi olarak verilmiştir. İkinci yaklaşımda ise; yüz üzerinde düzgün dă̆ılmış noktalarda yerel şekil betimleyicileri hesaplanmıştır. Hesaplanan yerel şekil betimleyiciler arasından tanıma süreci için ayırıcı olan seçilmiş ve üç boyutlu yüz tanıma için kullanılmıştır.

İki yaklaşım da genel kabul görmüş FRGCv2.0 veritabanı ve deney protokolü ile test edilmiştir. Raporlonan sonuçlar literatürdeki sonuçlardan daha iyidir. İfadesiz ve ifadeli yüzler için de tanıma performansları raporlanmıştır.

Anahtar Kelimeler: Üç boyutlu yüz tanıma, Şekil Haritaları, SIFT,SURF 


\section{ACKNOWLEDGMENTS}

It is a pleasure for me to express my sincere gratitude to my thesis supervisor Prof.

Dr. Uğur Halıcı for her belief, patience, encouragement and guidance throughout the study.

I would also like to thank the members of my thesis committee, Prof. Dr. Kemal Leblebicioğlu and Asst.Prof.Dr. Didem Gökçay for their valuable contributions. 


\section{TABLE OF CONTENTS}

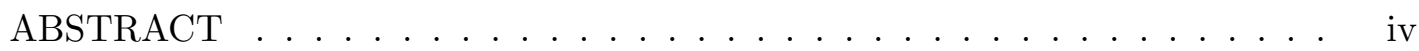

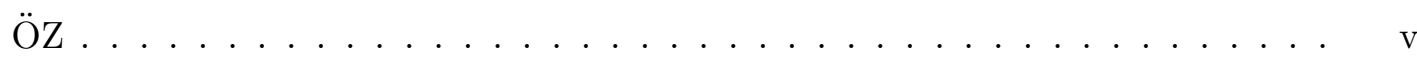

ACKNOWLEDGMENTS ........................... vi

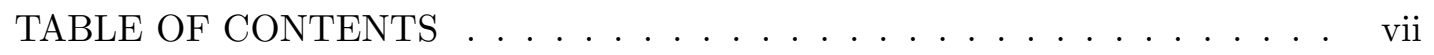

LIST OF TABLES . . . . . . . . . . . . . . . . . . . xi

LIST OF FIGURES . . . . . . . . . . . . . . . . . x xiii

CHAPTERS

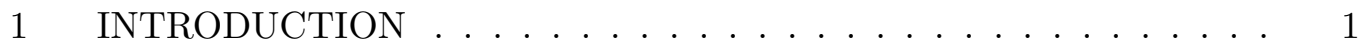

1.1 Biometric Recognition . . . . . . . . . . . . . . 1

$1.2 \quad$ Motivation for Research . . . . . . . . . . . . . . . 3

$1.3 \quad$ Contribution of the Thesis . . . . . . . . . . . . 4

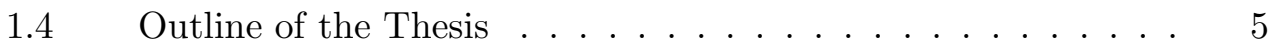

2 LITERATURE REVIEW . . . . . . . . . . . . . . . . 6

$2.1 \quad 3$ D Face Recognition $\ldots \ldots \ldots \ldots$

2.1.1 Holistic Approaches . . . . . . . . . . . . . . 6

2.1.2 Local Feature Based Approaches . . . . . . . . 9

2.1.3 Model Based Approaches . . . . . . . . . . . . . . . 10

2.1.4 Multi-modal Approaches . . . . . . . . . . 11

2.1.5 3D Face Recognition vs 2D Face Recognition . . . 11

3 AUTOMATIC 3D FACE RECOGNITION USING UNIFORMLY DISTRIBUTED SHAPE DESCRIPTORS . . . . . . . . . . . . . . 13

3.1 Overview of the proposed 3D face recognition system $\ldots \ldots 13$

$3.2 \quad$ Preprocessing of 3D Face Data . . . . . . . . . . . . 14

3.2.1 Smoothing . . . . . . . . . . . . 14 
3.2.3 Uniform Resampling and Cropping . . . . . . . . 15

$3.3 \quad$ Feature Generation . . . . . . . . . . . . . . 16

3.3.1 3D Shape Maps . . . . . . . . . . . . . . . 16

3.3.2 Uniformly Distributed Local Shape Descriptors for 3D Face Recognition . . . . . . . . . . . . . . . 21

3.3.3 2D Feature Descriptors applied on 3D Shape Maps . 21

3.4 Dimension Reduction . . . . . . . . . . . . . . . 25

3.5 Classification ........................ 25

3.6 Computational Complexity of the Proposed 3D Face Recognition System . . . . . . . . . . . . . 2 26

3.6.1 Computational Complexity of the Preproccesing Stage 26

3.6.2 Computational Complexity of the Differential Map Generation Stage . . . . . . . . . . . . 26

3.6.3 Computational Complexity of the Descriptor Calculation Stage . . . . . . . . . . . . . . 27

3.6.4 Computational Complexity of the LDA Stage (TRAINING $\ldots \ldots \ldots \ldots \ldots \ldots$

3.6.5 Computational Complexity of the LDA Stage (TESTING) . . . . . . . . . . . . . . 27

3.6.6 Computational Complexity of the Classification Stage 28

3.6.7 Computational Complexity of the Training . . . . . 28

3.6.8 Computational Complexity of the Testing . . . . . 29

4 GENERIC MODEL SUPPORTED 3D FACE RECOGNITION . . . . 30

$4.1 \quad$ Introduction $\ldots \ldots \ldots \ldots$

$4.2 \quad$ Annotated Face Database . . . . . . . . . . . . . . . 30

4.3 Manual Annotation of Facial Feature Points . . . . . . . . . 31

4.3.1 Annotated Facial Feature Points . . . . . . . . . . . 31

4.3.2 Interactive Tool for Annotation Facial Feature Points 34

4.3.3 Updating Facial Feature Points . . . . . . . . . 38

4.4 Preprocessing of 3D Face Data . . . . . . . . . . . 40

$4.5 \quad$ Generic Model Fitting . . . . . . . . . . . . . . . . 40

4.5.1 Generic Face Model . . . . . . . . . . . . . . . . . 40 
4.5.2 Radial Basis Function Fitting . . . . . . . . . 40

$4.5 .3 \quad$ Local Deformation . . . . . . . . . . . . . . 44

4.5.4 Fine Registration and Resampling . . . . . . . . . 44

4.6 Overview of the proposed generic model supported 3D face recognition system . . . . . . . . . . . . . 45

4.7 Local Shape Descriptors for Generic Model Supported 3D Face

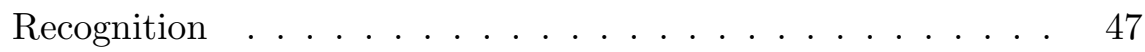

$4.8 \quad$ Feature Generation . . . . . . . . . . . . . . 47

4.9 Dimension Reduction and Classification . . . . . . . . . . . 50

5 EXPERIMENTAL RESULTS . . . . . . . . . . . . . . 51

$5.1 \quad$ FRGC Database and Experiment Protocol . . . . . . . . . 51

$5.1 .1 \quad$ FRGC Database . . . . . . . . . . . . . 51

5.1.2 FRGC Experiment Protocol . . . . . . . . . . 52

$5.2 \quad$ Baseline Experiments . . . . . . . . . . . . . . . . 52

5.2.1 Principal Component Analysis on Range Images (RANGEPCA $\ldots \ldots \ldots \ldots \ldots \ldots \ldots \ldots$

5.2.2 Linear Discriminant Analysis on Range Images (RANGELDA $\ldots \ldots \ldots \ldots \ldots \ldots \ldots$

5.2.3 Principal Component Analysis on Shape Maps (MAPPCA $\ldots \ldots \ldots \ldots \ldots \ldots$

5.2.4 Linear Discriminant Analysis on Shape Maps (MAPLDA $\ldots \ldots \ldots \ldots \ldots \ldots$. . . . . . . . . . . 64

5.3 Experimental Results for Automatic 3D Face Recognition Using Uniformly Distributed Shape Descriptors . . . . . . . . . . 64

5.3.1 Evaluation of different shape maps and descriptor sizes (UNI-EVA) . . . . . . . . . . . . . . . 64

5.3.2 Combined Feature Experiment with 5 Selected Shape Map - Descriptor Combinations (UNI-CF5D) . . .

5.3.3 Combined Feature Experiment with Multi Shape Map - Multi Scale SIFT Descriptors (UNI-CFMD) . . .

5.3.4 Reduced Feature Locations Experiment with 5 Selected Shape Map - Descriptor Combinations (UNIRF-CF5D) . . . . . . . . . . . .

5.3.5 Reduced Feature Locations Experiment with Multi Shape Map-Multi Scale SIFT Descriptors (UNI-RFCFMD) . . . . . . . . . . . . . 82 
5.3.6 Spatial Feature Selection with Sequential Forward Selection (SFS) Algorithm . . . . . . . . . . 86

5.3.6.1 Single Region Performance on FRGCv1.0 (UNI-SR-FRGC1-11fold) . . . . . . 86

5.3.6.2 Multi Region Performance on FRGCv1.0 (UNI-SFS-FRGC1-11fold) . . . . . 89

5.3.6.3 Single Region Performance on FRGCv2.0 (UNI-SR-FRGC2) . . . . . . . . . . 91

5.3.6.4 Multi Region Performance on FRGCv2.0 (UNI-SFS-FRGC2) . . . . . . . . . 92

5.4 Experimental Results for Generic Model Supported 3D Face Recognition . . . . . . . . . . . . . . . 94

5.4.1 Evaluation of different shape maps and descriptor sizes (GEN-EVA) . . . . . . . . . . . . . . . 94

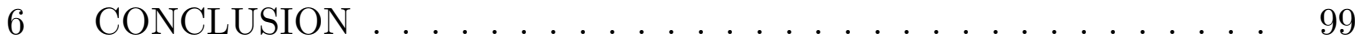

$6.1 \quad$ Summary . . . . . . . . . . . . . . . . . . 99

6.2 Discussion ...................... 100

$6.3 \quad$ Future Work . . . . . . . . . . . . . . . . . 101

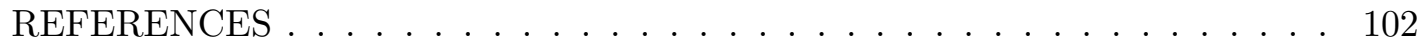

APPENDICES

A GENERIC FACE MODEL . . . . . . . . . . . . . 106

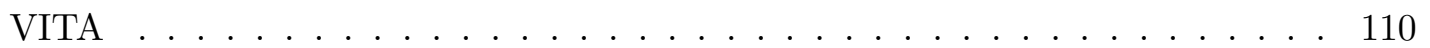




\section{LIST OF TABLES}

\section{TABLES}

Table 4.1 Manually Marked Facial Feature Points . . . . . . . . . . . . . . . . 34

Table 4.2 Manually Marked Facial Feature Points (continued) . . . . . . . . . 36

Table 5.1 Detection Rates for All vs All Experiment at 0.001 False Alarm Rate of Baseline 3D Face Recognition System with Shape Map + PCA (MAP-

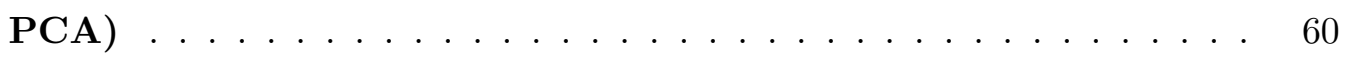

Table 5.2 Detection Rates for All vs All Experiment at 0.001 False Alarm Rate of Baseline 3D Face Recognition System with Shape Map + LDA (MAP-

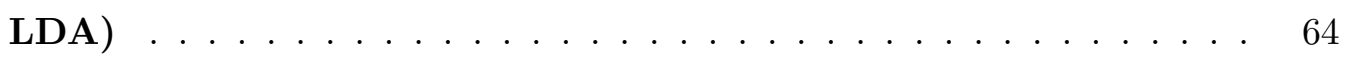

Table 5.3 Mean Detection Rates with Standard Deviations for All vs All Experiment at 0.001 False Alarm Rate of Automatic Model Supported 3D Face Recognition System for 457, 229 and 153 feature points (UNI-EVA) . . . 70

Table 5.4 Verification Results Using the All vs. All Data Set, at 0.1 Percent FAR 78

Table 5.5 Verification Results Using the ROC III Experiment, at 0.1 Percent

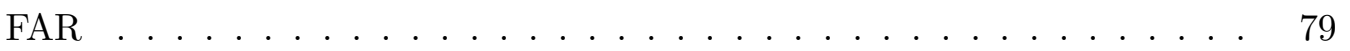

Table 5.6 Rank-1 Identification Results Using Earliest Scan as Gallery, Remain-

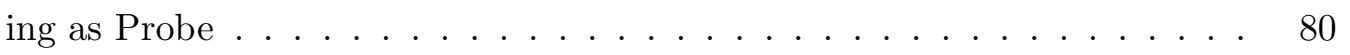

Table 5.7 Mean Detection Rates for All vs All Experiment at 0.001 False Alarm Rate with Single Face Region (Shape Index Map, SIFT Feature with 8mm width) on FRGCv1.0 Dataset (11-FOLD) (UNI-SR-FRGC1-11fold) . .

Table 5.8 Mean Detection Rates for All vs All Experiment at 0.001 False Alarm Rate with Multiple Face Regions Selected by SFS Algorithm (Shape Index Map, SIFT Feature with 8mm width) on FRGCv1.0 Dataset (11-FOLD) (UNI-SFS-FRGC1-11fold) 
Table 5.9 Detection Rates for All vs All Experiment at 0.001 False Alarm Rate with Single Face Region (Shape Index Map, SIFT Feature with 8mm width) on FRGCv2.0 Dataset (UNI-SR-FRGC2) . . . . . . . . . . . . . . . . 91

Table 5.10 Detection Rates for All vs All Experiment at 0.001 False Alarm Rate with Multiple Face Regions Selected by SFS Algorithm (Shape Index Map, SIFT Feature with 8mm width) on FRGCv2.0 Dataset (UNI-SFS-FRGC2) 92

Table 5.11 Detection Rates at 0.001 False Alarm Rate of Generic Model Supported 3D Face Recognition System for Different Sized Local Descriptors and Different Shape Maps (GEN-EVA) . . . . . . . . . . . . . 95 


\section{LIST OF FIGURES}

\section{FIGURES}

Figure 1.1 Verification Scenario . . . . . . . . . . . . . . . . . 2

Figure 1.2 Identification Scenario f . . . . . . . . . . . . . . . . 3

Figure 3.1 Block diagram of the proposed 3D face recognition system . . . . . 14

Figure 3.2 Normal planes and principal curvatures of a surface $[46] \ldots \ldots$

Figure 3.3 Sample shape maps ( SI, C, H and K maps) . . . . . . . . . . . . . 21

Figure 3.4457 nonuniformly distributed locations on 400x320 uniform grid . . 22

Figure 3.5 SIFT Descriptors calculated on shape index map at 457 uniformly distributed locations . . . . . . . . . . . . . . . . . . . 24

Figure 4.1 Sample 3D Face Scan in FRGC Database (front view) . . . . . . . 31

Figure 4.2 Sample 3D Face Scan in FRGC Database (side view) . . . . . . . . 32

Figure 4.3 Sample 2D Face Image accompanying the 3D scan in FRGC Database 33

Figure 4.4 Facial Animation Points ISO 14496 Codes $[27] \ldots \ldots$. . . . . . . 35

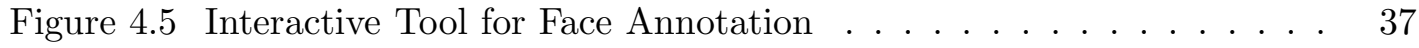

Figure 4.6 Face recording having inconsistency between 2D and 3D images . . 38

Figure 4.7 Sample annotated face recording $\ldots \ldots$. . . . . . . . . . . . 39

Figure 4.8 Generic Face Model (Front View) . . . . . . . . . . . . . . . . . . 41

Figure 4.9 Generic Face Model (Side View) . . . . . . . . . . . . . . . . . . . 42

Figure 4.10 Deformation Vectors (Front View) . . . . . . . . . . . . . . . 44

Figure 4.11 Deformation Vectors (Side View) . . . . . . . . . . . . . 45

Figure 4.12 Block diagram of the proposed generic model supported 3D face

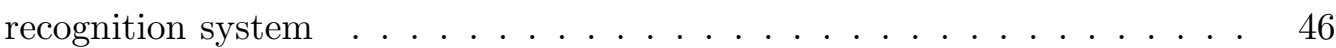


Figure 4.13 SIFT Descriptors calculated on shape index map at 549 nodes of

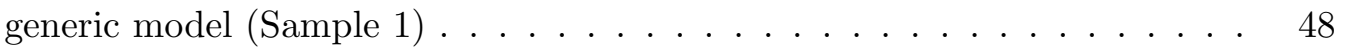

Figure 4.14 SIFT Descriptors calculated on shape index map at 549 nodes of generic model (Sample 2) . . . . . . . . . . . . . . . . . .

Figure 5.1 Block diagram of the simplified 3D face recognition system for RANGE-PCA and RANGE-LDA baseline experiments . . . . . . . .

Figure 5.2 ROC curves for PCA applied on range images (All vs All, ROC3)

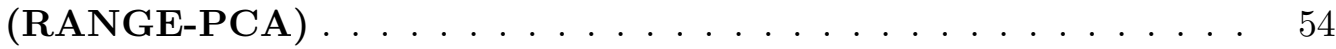

Figure 5.3 ROC curves for PCA applied on range images(Neutral vs Neutral, Neutral vs Non-Neutral, Non-Neutral vs Non-Neutral) (RANGE-PCA) . 55

Figure 5.4 Cumulative Match Characteristics for PCA applied on range im$\operatorname{ages}($ RANGE-PCA $) \ldots \ldots \ldots \ldots \ldots \ldots$

Figure 5.5 ROC curves for LDA applied on range images (All vs All, ROC3)

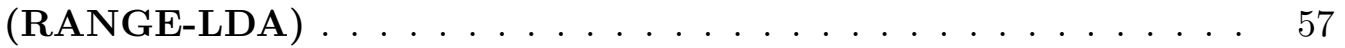

Figure 5.6 ROC curves for LDA applied on range images(Neutral vs Neutral, Neutral vs Non-Neutral, Non-Neutral vs Non-Neutral) (RANGE-LDA) . 58

Figure 5.7 Cumulative Match Characteristics for LDA applied on range im$\operatorname{ages}($ RANGE-LDA $) \ldots \ldots \ldots \ldots \ldots$

Figure 5.8 Block diagram of the simplified 3D face recognition system for MAPPCA and MAP-LDA baseline experiments . . . . . . . . . . . . 60

Figure 5.9 ROC curves for SI, C (top row) and H, K (bottom row) Shape Maps with PCA (All vs All, ROC3) (MAP-PCA) . . . . . . . . . . . . 61

Figure 5.10 ROC curves for SI, C (top row) and H, K (bottom row) Shape Maps with PCA (Neutral vs Neutral, Neutral vs Non-Neutral, Non-Neutral vs Non-Neutral) $($ MAP-PCA) . . . . . . . . . . . . . . . . . . 62

Figure 5.11 Cumulative Match Characteristics for SI, C (top row) and H, K (bottom row) Shape Maps with PCA (MAP-PCA) . . . . . . . . . .

Figure 5.12 ROC curves for SI, C (top row) and H, K (bottom row) Shape Maps with LDA (All vs All, ROC3) (MAP-LDA) . . . . . . . . . . . 
Figure 5.13 ROC curves for SI, C (top row) and H, K (bottom row) Shape Maps with LDA (Neutral vs Neutral, Neutral vs Non-Neutral, Non-Neutral vs Non-Neutral) $($ MAP-LDA) $\ldots \ldots \ldots 66$

Figure 5.14 Cumulative Match Characteristics for SI, C (top row) and H, K (bottom row) Shape Maps with LDA (MAP-LDA) . . . . . . . . . . 67

Figure 5.15 ROC curves for 5x229x128 features (All vs All, ROC3) (UNI-CF5D) 71

Figure 5.16 ROC curves for 5x229x128 features(Neutral vs Neutral, Neutral vs Non-Neutral, Non-Neutral vs Non-Neutral) (UNI-CF5D) . . . . . . . . . 72

Figure 5.17 Cumulative Match Characteristics for 5x229x128 features (UNI-

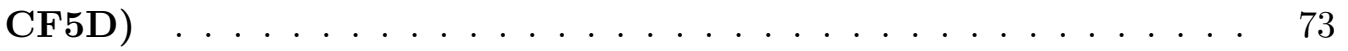

Figure 5.18 ROC curves for 5 (scales) x 4 (shape maps) x 229 (locations) x 128 (SIFT feature size) initial features (All vs All, ROC3) (UNI-CFMD) . . 74 Figure 5.19 ROC curves for 5 (scales) x 4 (shape maps) x 229 (locations) x 128 (SIFT feature size) initial features(Neutral vs Neutral, Neutral vs NonNeutral, Non-Neutral vs Non-Neutral) (UNI-CFMD) . . . . . . . . . 75 Figure 5.20 Cumulative Match Characteristics for 5 (scales) x 4 (shape maps) x 229 (locations) x 128 (SIFT feature size) initial features (UNI-CFMD) . 76 Figure 5.21 ROC curves for 5x345x128 features (All vs All, ROC3) (UNI-RF-

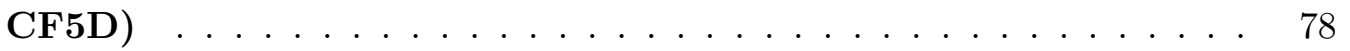

Figure 5.22 ROC curves for 5x345x128 features(Neutral vs Neutral, Neutral vs Non-Neutral, Non-Neutral vs Non-Neutral) (UNI-RF-CF5D) . . . . . . 79

Figure 5.23 Cumulative Match Characteristics for 5x345x128 features (UNI-

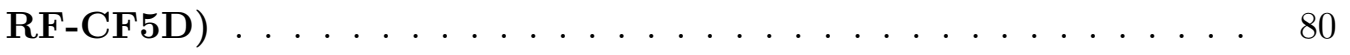

Figure 5.24 SIFT Descriptors calculated on shape index map at 345 uniformly distributed locations (mouth and chin regions excluded) . . . . . . . 81

Figure 5.25 ROC curves for 5 (scales) x 4 (shape maps) x 345 (locations) x 128 (SIFT feature size) features (All vs All, ROC3) (UNI-RF-CFMD) . . .

Figure 5.26 ROC curves for 5 (scales) x 4 (shape maps) x 345 (locations) x 128 (SIFT feature size) features(Neutral vs Neutral, Neutral vs Non-Neutral, Non-Neutral vs Non-Neutral ) (UNI-RF-CFMD) . . . . . . . . . . . . 
Figure 5.27 Cumulative Match Characteristics for 5 (scales) x 4 (shape maps) x 345 (locations) x 128 (SIFT feature size) initial features (UNI-RF-CFMD) 85

Figure 5.28 26 Face Regions with corresponding feature locations(top), region numbers (center), number of feature locations in each face region(bottom)

Figure 5.29 Mean Detection Rate vs Number of Regions Selected by SFS on FRGCv1.0 Dataset (11-FOLD) (UNI-SFS-FRGC1-11fold) . . . . . . . 90

Figure 5.30 Detection Rate vs Number of Regions Selected by SFS on FRGCv2.0 Dataset (UNI-SFS-FRGC2) . . . . . . . . . . . . . . . . . . . 93

Figure 5.31 ROC curve for 549x128 features on generic model (All vs All) (GEN-

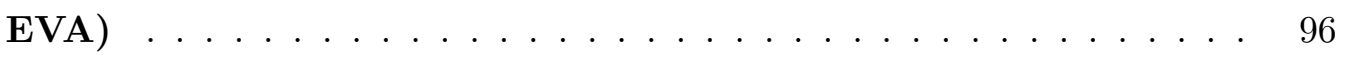

Figure 5.32 ROC curve for 549x128 features on generic model (Fall2003 vs Spring2004) (GEN-EVA) . . . . . . . . . . . . . . . . . 96

Figure 5.33 ROC curve for 549x128 features on generic model (Neutral vs Neu-

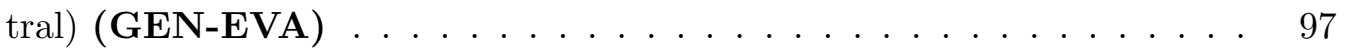

Figure 5.34 ROC curve for 549x128 features on generic model (Neutral vs NonNeutral) $($ GEN-EVA) . . . . . . . . . . . . . . . . . . . . . 97

Figure 5.35 ROC curve for 549x128 features on generic model (Non-Neutral vs Non-Neutral) (GEN-EVA) . . . . . . . . . . . . . . . . . . . . . 98 


\section{CHAPTER 1}

\section{INTRODUCTION}

\subsection{Biometric Recognition}

The word biometrics includes two ancient Greek words bios, meaning life, and metron which stands for measure. Biometric recognition is the study of the recognition of people based on their distinctive anatomical characteristics. Significant types of biometrics can be listed as follows: fingerprint, iris, face, retina, vein, voice, and hand geometry. If the requirements such as universality, distinctiveness, permanence and collectability are satisfied, it enables any desired human physiological characteristic to be used as a biometric characteristic. Universality can be defined as the characteristic each individual should have. Distinctiveness refers to the necessary difference of characteristics between any two individuals. The permanence of a characteristic is its invariance over time, and as the last requirement; collectability refers to the ability to be measured quantitatively[28].

Performance, which can be defined as the recognition accuracy and speed; acceptability, which refers to how much a particular biometric is accepted in every day life; and circumvention, which is a measure of how readily the system can be deceived, should also be considered in biometric systems.

A biometric system is a special case of a pattern recognition system, which works by first acquiring biometric data, then using this acquired data to form a feature set and then finally comparing this feature set with the template set. Biometric systems are usually used in two different scenarios : subject verification and subject identification. In subject verification scenario (Figure 1.1), the user claims his/her identity and 
authentication system verifies or rejects the identity. This method requires one-toone matching of the biometric data. Verification scheme needs a threshold value to decide if the given biometric record matches with that of the claimed identity. In order to avoid threshold dependent performance measurement, verification performance of biometric systems are almost always reported by displaying the receiver operator characteristics curves (ROC curves). ROC curves are widely used in signal detection context and they are obtained by plotting true detection rate versus false alarm rate by changing the threshold from minimum to maximum possible values.

Subject identification scenario (Figure 1.2), on the other hand, does not require an identity to be claimed, the subject just presents the biometric of his/her own. Biometric system identifies the subject from the given biometric. This method requires one-to-many matching of the biometric data.

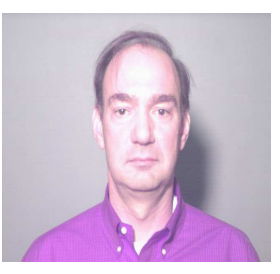

Biometric Record

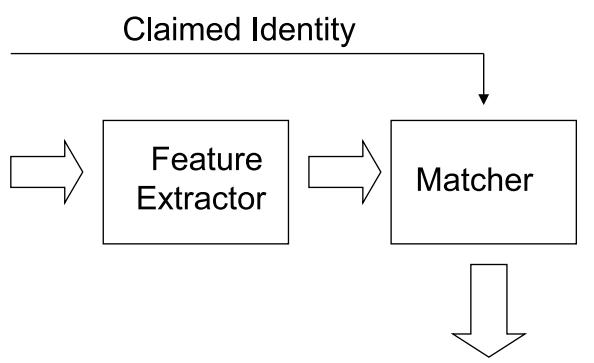

True/False

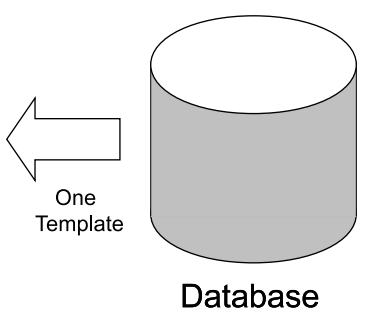

Database

Figure 1.1: Verification Scenario

Face is very popular as a biometric, since it has many advantages like being nonintrusive and accurate. 2D face images are very easy to acquire and have been widely used as biometric data. However it is clear that a 2D face image depends on lightning, 


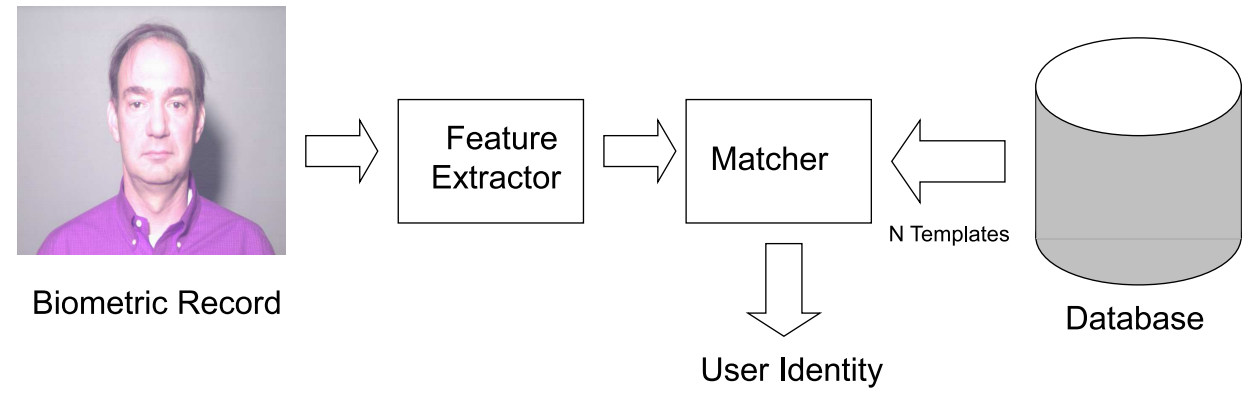

Figure 1.2: Identification Scenario

surface albedo and head pose. These shortcomings motivated researchers to move to 3D face recognition. 3D face recognition uses the discriminative shape information to make a decision about the identity.

\subsection{Motivation for Research}

Performance of both 2D and 3D face recognition systems relies on the quality of face record and recognition performance of the proposed algorithm. A face record is easily altered by head pose, expressions and current recording technology. A successful recognition algorithm should tolerate these variations. It is obvious that, if these variations are sufficiently large between training and test images, it gets hard to handle these variations.

Another problematic issue with face recognition studies is the relatively small number of training images. This issue is usually addressed as small sample size problem in the 
literature. Whichever data representation and feature generation approach is used, the feature vectors representing the face records come out to be high dimensional. Small number (almost always one indeed) of training samples is a major problem for locating the boundaries of a person specific hyper-volume in high dimensional feature space.

The main goal of this thesis is to address the problem of 3D face recognition taking facial expressions and data acquisition artifacts into account. Our approach is based on calculation of robust local shape descriptors and extracting discriminative information from these descriptors. Shape descriptors should be informative enough to satisfy the needs of the recognition process and robust enough to handle the variation due to facial expressions and acquisition artifacts.

\subsection{Contribution of the Thesis}

In this study, we have investigated the application of local shape descriptors without interest point detectors for the 3D face recognition problem. Local shape descriptors are calculated on the differential shape maps of the range data. Since no interest point detectors are used, we have to deal with the locations of the local shape descriptors. For this problem, two approaches are considered. In the first approach, a fixed uniform grid is prepared and SIFT/SURF descriptors are calculated on the differential shape map at the locations defined by fixed uniform grid. In the second approach, a generic face model is deformed to fit to the $3 \mathrm{D}$ face data and local shape descriptors are calculated at the fiducial points of the generic face model. For both of the approaches, LDA based dimension reduction is applied to the calculated shape descriptors. Faces are compared in this subspace with reduced number of dimensions by the help of a cosine metric. To the best of our knowledge, local shape descriptors (differential shape maps + SIFT/SURF) with subspace projection is applied to the 3D face recognition problem for the first time. The contribution of this classification scheme for the $3 \mathrm{D}$ face recognition problem is to combine the discriminative power of the linear discriminant analysis with the robustness of SIFT/SURF descriptors applied on differential shape maps without employing interest point detectors. 


\subsection{Outline of the Thesis}

In this thesis, we propose two different types of 3D face recognition methods. The first method is the generic model supported face recognition with local shape descriptors. In this method, a generic face mesh is fitted to the subject's face and local shape descriptors are calculated at the nodes of the generic face mesh. Subjects are recognized by the classifier using the discriminative information at local shape descriptors. The second method is the automatic 3D face recognition method using local shape descriptors, which are uniformly distributed locations across the subjects' faces. Local shape descriptors at uniformly distributed locations are fed to the classifier stage and recognition process is completed.

The thesis is organized as follows:

- In chapter 2 , we briefly describe the history of 3D face recognition. We note the recent developments in the face recognition literature.

- In chapter 3, the automatic 3D face recognition method using local shape descriptors, which are uniformly distributed across the face is proposed. Among the calculated shape descriptors the ones that are discriminative for the recognition process are selected and used for three dimensional face recognition.

- Chapter 4 presents the approach we have implemented for generic mesh fitting on the 3D face data. Later, the generic model supported 3D face recognition method is explained. 3D face recognition approach with local shape descriptors located on the nodes of the generic model mesh is given.

- In Chapter 5, performances of the automatic 3D face recognition method and generic model supported 3D face recognition method evaluated with FRGC (face recognition grand challenge) v2.0 3D face database and the experiment protocol[38].

- Finally in chapter 6 , we state the conclusions and mention possible future research directions. 


\section{CHAPTER 2}

\section{LITERATURE REVIEW}

\subsection{D Face Recognition}

This chapter will focus on the literature about 3D face recognition. Current approaches for 3D feature extraction and classification will be provided. We separate the literature review into five distinctive categories: i) Holistic Approaches, which recognizes face by matching the whole face, ii)Local feature based approaches, which perform face recognition by using the similarity of local features, iii) Model Based $A p$ proaches, which use recognition methods that are supported by analytical models of face, iv)Multi-modal approaches, which use both 2D (intensity image) and 3D (range image) modalities to get face recognition systems with improved performance and v)3D Face Recognition vs 2D Face Recognition section which gives a comparison between the $2 \mathrm{D}$ and $3 \mathrm{D}$ modalities in the face recognition context.

\subsubsection{Holistic Approaches}

Holistic Approaches of 3D face recognition treats the face as a whole and focus on global similarity of the faces. One of the first holistic 3D recognition studies is given in [24]. Both principal component analysis and independent component analysis are used for dimension reduction of 3D face data. They perform identification with nearest neighbor rule using euclidean metric on the reduced subspace. System performance is tested on relatively small training and test sets. In [44], 3D spherical gabor filters are proposed as the main tool for holistic feature extraction. In this study least trimmed 
square Hausdorff distance is used to overcome problems such as self-occlusion, distortions etc. This promising method however is tested on a small-sized set. In [11], to compute three dimensional canonical surfaces geometric moments up to fifth order are used. They tested their system on a 3D face database containing 220 faces of 30 subjects. In [37], an annotated morphable model in accordance with elastic deformation constraints is fitted to the subject's face. They use $L^{1}$ metric for measuring distances between gallery and probe images and report their performance on the FRGC data corpus [38].

Facial curve matching is one of the approaches used for 3D face recognition. Haar et al. [42] states a framework that is capable of profile and contour based matching. They propose four types of contour features: G-curves, $C$-curves, $X Y$-curves, $Z$-curves. $G$ curves stand for approximated shortest geodesic path of $r \mathrm{~mm}$ over the surface to the origin. C-curves, on the other hand, are samples with length of $r \mathrm{~mm}$ over the profile which is in radial direction emanating from the origin. XY-curves represent contours which consist of points which have circular projections on XY plane. These circles' centers are located at the origin and they have a radius of $r$. Z-curves are the intersections of the $3 \mathrm{D}$ face surface with $\mathrm{Z}=r$ planes. They built their framework on these sets of curves and distance metric used for similarity measurement.

Spherical functions are also used for 3D face recognition. Llonch et al. [40] addresses such an approach using simultaneous sparse approximation on sphere. Their approach mainly depends on capturing salient shapes of 3D face surface with a small number of spherical functions. In their implementation, they use 2D Gaussian functions on 3D sphere as the generating for atoms(i.e. spherical functions which are the elements of dictionary). They use simultaneous matching pursuit algorithm to find the atoms which model the 3D faces with smallest residual values. An optional linear discriminant analysis stage is placed to further reduce the number of dimensions and to increase the discriminative power of the resultant feature vector. Performance of the proposed system is tested on FRGC v1.0 database and shown to be competitive with the state-of-the-art systems. Proposed method is especially important for representing the most of the shape information with a very small number coefficients. Performance 
of the method may be even increased more with better choices of representing functions.

A 3D facial image recognition method based on curvatures is given in [32]. This paper presents a multi-stage approach based on curvatures of range image. Proposed method starts with extraction of four rectegular regions, namely, eye, nose, cheek and mouth regions. In the second stage, minimum, maximum, gaussian and mean curvatures of these regions. Second stage of the proposed algorithm consists of PCA+LDA to reduce the dimensionality of the output data of the previous stage. In the third and last phase, membership grades and fuzzy integrals are used to generate output classes. They test the proposed method's performance on a database of 592 images acquired by themselves.

Surface feature maps are by used by Heseltine et al. [23] for 3D face recognition. They first do the pose normalization and pan, tilt, roll correction on the 3D facial scans. In the second stage, they generate the following feature maps: Depth map, horizontal and vertical gradient maps at two different scales, laplacian map, sobel gradient map in $X$ and $Y$ directions, curvature type map, convexity and concavity maps. In the next phase, linear discriminant analysis is done to extract the discriminative part of the feature maps. The proposed system is tested on a database of 1470 depth maps of 230 people. Best performance figure is $\% 7.2$ false acceptance/rejection rate at equal error rate.

There are various types representations of 3D face data and feature extraction methods from the face data. Gokberk et al. [22] explores many types of representations (point clouds, depth images, surface normals, curvatures, voxels) and many types of feature extraction methods (shape index, nonnegative matrix factorization, independent component analysis etc.) for 3D face recognition. They perform extensive set of experiments on FRGC v2.0 database and they conclude that representation is more important if the training set is small. On the other hand type of features is more critical when the size of the training database gets larger. Another conclusion 
they get through the experiments is that fusion of experts has positive effect on the performance but one should carefully normalize the experts' scores to be fused.

\subsubsection{Local Feature Based Approaches}

Local Feature Based Approaches find and use local distinctive properties of the faces. Log-gabor templates are used in [14] to divide range images into regions where effects of deformations due to expressions are considered. They merge the classification results in the score level and check the system's performance on FRGC database. An elastic graph is used in [26] for finding fudicial facial landmarks and for generating features. They display all 2D, 3D and 2D+3D fusion performances separately. In [13], multiple regions around the nose are used for 3D face recognition. This article is important in indicating the importance of the nose region in the context of $3 \mathrm{D}$ face recognition. The shape of nose region is robust to many expressions and therefore has valuable descriptive information. In [19], 38 predefined coordinates in X-Y plane are used to locate local features. A predefined sphere radius in conjugate with $(\mathrm{X}, \mathrm{Y})$ coordinates defines the sphere containing the local surface patches. These surface patches are independently registered with Iterative Closest Point (ICP) algorithm and registration error is used as a dissimilarity metric. Final decision is made by region committee voting approach. Faltemier et al.[18] proposes a multi-instance enrollment scheme for 3D face recognition. They test their approach on a 3D database containing 13450 scans of 888 subjects. This large database is a superset of FRGC v2.0 database and rich in terms of expressions They show the positive effect of multi-instance enrollment by rich set of experiments. The face recognition method of this study is based on the work at [19].

McCool et al. [35] uses divide face range image into overlapping blocks for 3D face verification. Hence they obtain independent range patches in these blocks. 2D discrete cosine transform (DCT) applied onto the blocks and corresponding DCT coefficients are calculated. The calculated coefficients are then used for face verification. Proposed approach treats each part as independent observations and spatial relationship block is discarded. The authors' claim that proposed method may be improved by extactiong spatial information as well. 
Registration of 3D faces is an important preprocessing phase of 3D face recognition systems. Registration is usually considered as global problem, however there are studies that handle registration problem locally. A recent attempt for regional registration is given in [3]. Alyuz et al.[3] build average face and regional models to initiate their method. Following the registration of surface patches to corresponding average face models, curvature based 3D shape descriptors are calculated. These descriptors are fed into independent LDA classifiers and outputs with reduced number of dimensions are fused, and finally classification is completed by 1-nearest neighbor classifier. They report $97.51 \%$ rank- 1 registration rate and $86.09 \%$ verification rate at $0.1 \%$ false alarm rate for ROC3 expreriment on FRGC v2.0 database.

Registration of two range images is sometimes interpreted as a measure of similarity. Queirolo et al. citeQueirolo2010 is one of the recent studies that approaches the 3D face recognition problem in this manner. They use simulated annealing for the registration of $3 \mathrm{D}$ faces and surface interpenetration measure as the similarity metric. They divide the face into nine sectors with a rectangular grid and they prefer to use the three sectors in the top row (forehead region) and the center sector (nose region) for face matching. Proposed face recognition system is tested on FRGC v2.0 database. Rank-1 registration rate is $98.4 \%$ for earliest scan vs remaining test and $96.5 \%$ is reported as verification rate at $0.1 \%$ false alarm rate for All-vs-All expreriment.

\subsubsection{Model Based Approaches}

Model Based Approaches are the face recognition methods that are supported analytical models of the face. Haar et al. [41] proposes a 3D face recognition method supported by a statistical face deformation model. They try to optimize the shape parameters to get a 3D face model that fits into 3D scan data. They divide the face into predefined components and blend these components to achieve a better fitting of the deformation model. Performed experiments show $97.5 \%$ correct identication rate for 876 queries of UND face data set. A fully automatic 3D recognition system with 3D facial model support is proposed by Kakadiaris et.al [29]. They start with expression- 
invariant deformable model fitting to 3D face scan. Deformed model is transformed to a $2 \mathrm{D}$ geometry image and obtained $2 \mathrm{D}$ image is expressed in wavelet domain. It is claimed that only a small portion of wavelet data captures most of the variations of 3D image and therefore enough for recognition purposes. The proposed system is tested on FRGC v2.0 database and \%97 verification rate is reported at $0.1 \%$ false alarm rate.

\subsubsection{Multi-modal Approaches}

Multi-modal Approaches use the information of both 2D intensity images and 3D shape. Local gabor features which do have flexibility on orientation and scale gained the attention of researchers working on face recognition. A successful implementation of gabor wavelets is given in [47]. A multi-modal approach, which use 2D and $3 \mathrm{D}$ face data simultaneously is proposed. The method starts with preprocessing $2 \mathrm{D}$ and 3D face data and preprocessed face data is cropped by the help of an elliptical mask. They select five scales and eight orientations and obtain forty different gabor wavelets. Therefore they have 475840 (5948 pixels in elliptical mask x 8 orientations x 5 scales x 2 modalities) dimensional face descriptor vectors. Taking into account curse of dimensionality, they propose a two stage approach for reducing the number of dimensions. In the first stage, subsampling (i.e. dimension reduction) is realized by LDA trained by a small subset of faces. In the second stage, adaboost learning is used for reducing dimensions. Final stage of the cascaded classifier is also built with an adaboost learner. The study reports $95.3 \%$ detection rate for ROC3 experiment at $0.1 \%$ false alarm rate, which represents a performance figure comparable to state-ofthe-art studies. One of the studies that use both 2D and 3D information is presented in [8] where central and lateral profiles of $2 \mathrm{D}$ and $3 \mathrm{D}$ data are extracted. They prefer score level fusion to overcome the effects of thresholding in decision level fusion.

\subsubsection{D Face Recognition vs 2D Face Recognition}

There are number of studies which compare $2 \mathrm{D}, 3 \mathrm{D}$ and $2 \mathrm{D}+3 \mathrm{D}$ modalities in many aspects such as performance, algorithm complexity and biometric acquisition. In Bowyer et al. [9], an extensive survey of 3D and 2D+3D face recognition approaches 
is given. They note three main challenges that 3D face recognition systems face. First of these challenges is the limited technology and acquisition quality of current 3D shape recording hardware. 3D sensors still have problems with ambient lighting, recording artifacts and user cooperation. Second challenge is designing better algorithms which are more tolerant of variations due to the pose, facial expression, eye-glasses and similar accessories. Final challenge is the computational effort required. They claim that three-dimensional face recognition usually requires more computational effort per match than 2D face recognition. Another study of Bowyer [10] focuses on the comparison of multimodal (2D, 3D, infrared and their combinations) facial biometric systems with multisample (multi-instance) supported system. This study is valuable especially because the $2 \mathrm{D}$, infrared, and 3D images of a subject were all acquired within couple of minutes at the same conditions. 2D and 3D images are 640 by 480 image resolution whereas infrared image is sized as 240 by 320 pixels. They find 3D modality is slightly better than $2 \mathrm{D}$ modality for rank-1-recognition rate. Infrared modality, on the other hand, has significantly lower rank-1-recognition tan that of for 2D or 3D modalities. Also it is noted that each of the multimodal combinations has improved performance with respect to the individual modalities. As expected, best result is reported with combination of all three modalities.

A detailed survey study about 2D and 3D face recognition is given in [2]. They claim 3D face recognition has the potential to increase the recognition performances of the 2D face recognition systems especially in case of pose, illumination and expression variations. Yet, 3D approaches still have the challenges with alignment of meshes and sensitivity of the 3D face acquisition. Detection of occlusive objects is addressed as an important issue and 3D recognition is reported to have more potential with the problem of occlusion. As a final remark, they point out that only multimodal approaches are robust enough to cope with the distortions in real world biometric problems. 


\section{CHAPTER 3}

\section{AUTOMATIC 3D FACE RECOGNITION USING UNIFORMLY DISTRIBUTED SHAPE DESCRIPTORS}

This chapter presents the proposed face recognition method based on uniformly distributed local shape descriptors. The chapter begins with giving an outline of the method, then preprocessing, feature generation and classification stages are explained in order.

\subsection{Overview of the proposed 3D face recognition system}

In figure 3.1, the block diagram of the proposed 3D face recognition system is displayed. The process starts with the acquisition of the 3D face data with a face scanner. In the next stage, 3D face data is registered and its spikes and holes are removed by smoothing. Preprocessing is finished with uniform resampling and cropping. Preprocessed 3D face data are fed into the next stage for calculation of differential shape parameters. These parameters are shape index, curvedness, mean and gaussian curvature. At this stage, we have 2D matrices of differential shape parameters representing the local shape information. At the next stage, SIFT and SURF operators are applied on these 2D matrices. Using SIFT and SURF operators allows us to summarize local shape information in terms of histograms. These histograms have sufficient information for describing shape characteristics of the subject's face. However, the dimensionality of the total feature vector is very high and it has redundant non-discriminative information as well as discriminative information. This problem has been resolved in the dimension reduction stage and high-dimensional feature vector is projected onto a 
discriminative low-dimensional subspace. Operation of the 3D face recognition system is completed with comparison of feature vectors at low dimensional subspace and a final decision is made on the subject's identity.

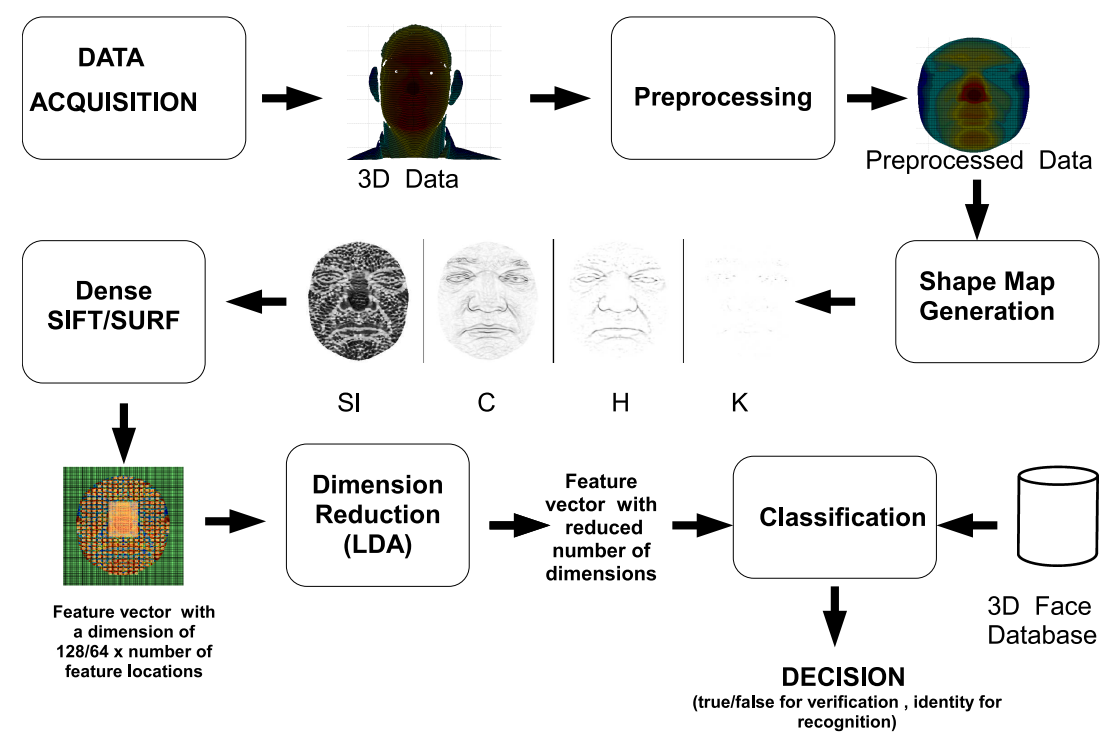

Figure 3.1: Block diagram of the proposed 3D face recognition system

\subsection{Preprocessing of 3D Face Data}

Preprocessing of biometric data is an essential part of the biometric recognition systems. We preprocess 3D face data to minimize the effects of the intrinsic noise of face scanner and to have normalized and registered 3D faces. Our preprocessing have four stages. These stages are smoothing, registration, uniform resampling and cropping. The details of these stages are given in the following.

\subsubsection{Smoothing}

For smoothing, 2D median filtering is applied to $\mathrm{Z}$ values which are representing the depth. This is done especially to remove the spikes due to the 3D scanner. Although median filtering removes the spikes, it may also unnecessarily suppress actual varia- 
tions on the data. Taking this fact into account, we apply modified median filtering process, which has the following steps:

- Label original data as original range.

- Apply 2D 5x5 median filter operator to original data and label output as filtered range.

- Calculate the absolute difference between original range and filtered range.

- For each point of the data grid, if the absolute difference is higher than 3 millimeters then the corresponding data from filtered range is used. If the absolute difference is lower than 3 millimeters, then the corresponding data from original range is used.

Such a filtering approach eliminates the spikes whereas conserves original data in regions without spikes.

\subsubsection{Registration}

Orientation correction is made on 3D face data after median filtering. In plane rotation is removed by positioning the symmetry axis of shape index map as applied in the work of Alyuz et al. [3]. Registration is completed by positioning the nose-tip at the origin. In order to find the nose tip, first the vertical strip, which has maximum symmetry value is searched and then the point with minimum range value on this strip is labeled as the nose-tip.

\subsubsection{Uniform Resampling and Cropping}

The registered 3D face image is interpolated and resampled at a uniform grid of 0.5 $\mathrm{mm}$ resolution both in $\mathrm{X}$ and $\mathrm{Y}$ directions. The uniform grid has a height of 400 pixels and a width of 320 pixels. $\left(y_{\min }=-99.5, y_{\max }=100, x_{\min }=-79.5, x_{\max }=80\right)$. 
Once the 3D faces have been registered and uniformly resampled, the points, which are inside the sphere with origin as the center (nose-tip) and $100 \mathrm{~mm}$ as radius are cropped and only these points are used for recognition purposes.

\subsection{Feature Generation}

3D shape recognition requires descriptors that are capable of describing local shape efficiently. We use a two phase approach:

- In the first phase, 3D shape maps of the face are calculated. These maps are Shape Index Map, Curvedness Map, Mean Curvature Map and Gaussian Curvature Map.

- In the second phase, local 2D descriptors are calculated on the shape maps. We apply scale invariant feature transform $(\operatorname{SIFT})[33,5]$ and speeded-up robust features(SURF) [4] descriptors on the shape maps calculated in the first phase.

\subsubsection{D Shape Maps}

Let $p$ be an arbitrary point on a differentiable surface in 3-dimensional Euclidean space. The unit vector emanating from the point $p$ and perpendicular to the surface is called the unit normal vector. A normal plane at $p$ is the one that contains the normal vector.

Intersection of the normal plane and the surface is a 3D curve. 3D curves in different normal planes may have different curvatures. The principal curvatures (Figure 3.2) at $p$, denoted $k_{\max }$ and $k_{\min }$, are the maximum and minimum values of these curvatures. Here the curvature of a curve is defined as the reciprocal of the radius of the osculating circle. Usual convention is to take the curvature to be positive if the curve turns in the same direction as the surface's chosen normal, and negative otherwise.

Mean and Gaussian Curvatures. Mean curvature, $H$, is the mean of principal curvatures [31] and is defined as in Equation 3.1. 


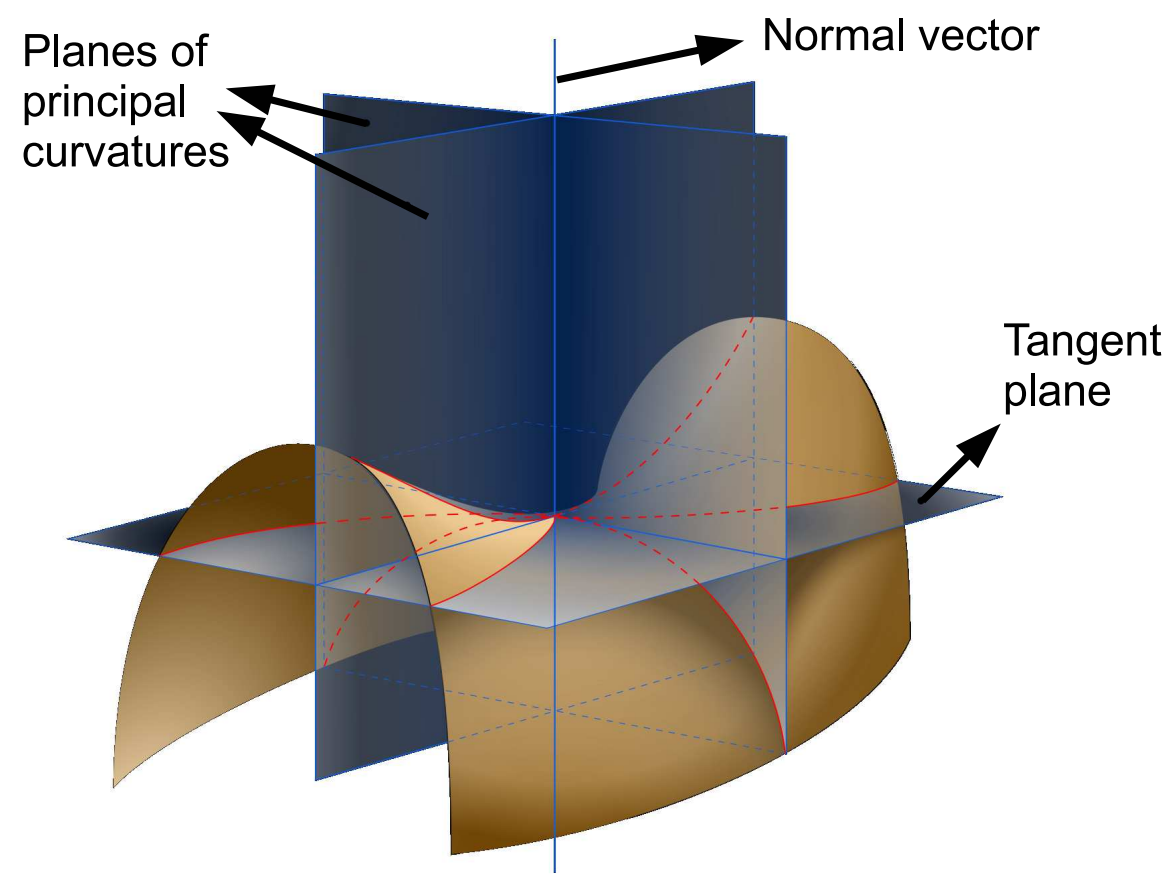

Figure 3.2: Normal planes and principal curvatures of a surface [46] 


$$
H=\frac{k_{\max }+k_{\min }}{2}
$$

where $k_{\max }$ and $k_{\min }$ are minimum and maximum principal curvatures as previously explained.

Gaussian curvature, K, is the product of principal curvatures [31]. Formally, it is defined as in Equation 3.2.

$$
K=k_{\max } \cdot k_{\min }
$$

Shape Index and Curvedness. The shape index, SI, is a metric which describes local shape topography in terms of principal curvatures. Formally, it is defined as in equation 3.3 [31]. We should note that, the shape index is independent of the scale.

$$
S I=\frac{-2}{\pi} \arctan \left(\frac{k_{\max }+k_{\min }}{k_{\max }-k_{\min }}\right)
$$

The curvedness, C, also describes local shape topography in terms of principal curvatures [31]. Formally, it is defined as in Equation 3.4. Unlike the shape index, the curvedness is strictly dependent on the local scale.

$$
C=\sqrt{\left(\frac{k_{\max }^{2}+k_{\min }{ }^{2}}{2}\right)}
$$

Generation of Shape Maps with Local Surface Differentials. Differential shape parameters such as shape index, curvedness, mean curvature and gaussian curvature are all defined in terms of principal curvatures, and they can be computed by using local differentials of the surface[31]. Let us consider a differentiable surface patch $\sigma(u, v): U \rightarrow \Re^{3}$. First fundamental form of the surface patch is given in Equation 3.5 


$$
d s^{2}=E d u^{2}+2 F d u d v+G d v^{2}
$$

where $d s$ is the differential curve length and $E, F, G$ can be calculated as follows:

$$
\begin{gathered}
E \quad: \vec{\sigma}_{u} \cdot \vec{\sigma}_{u} \\
F \quad: \vec{\sigma}_{u} \cdot \vec{\sigma}_{v} \\
G \quad: \vec{\sigma}_{v} \cdot \vec{\sigma}_{v}
\end{gathered}
$$

Second fundamental form of the surface patch is given in Equation 3.9 and $\vec{n}, \mathrm{~L}, \mathrm{M}$, $\mathrm{N}$ can be calculated as follows:

$$
\Delta=\frac{1}{2}\left(L d u^{2}+2 M d u d v+N d v^{2}\right)
$$

where $\Delta$ is the deviation of $\sigma$ from the tangent plane

$$
\begin{gathered}
\vec{n} \quad: \frac{\vec{\sigma}_{u} \times \vec{\sigma}_{v}}{\left\|\vec{\sigma}_{u} \times \vec{\sigma}_{v}\right\|} \\
L \quad: \vec{\sigma}_{u} \cdot \vec{n} \\
M \quad: \vec{\sigma}_{u} \cdot \vec{n} \\
N \quad: \vec{\sigma}_{v} \cdot \vec{n}
\end{gathered}
$$

Let $\mathbb{M}_{1}$ and $\mathbb{M}_{2}$ be the matrix forms of the first and second fundamental forms given in Equation 3.14: 


$$
\mathbb{M}_{1}=\left(\begin{array}{cc}
E & F \\
F & G
\end{array}\right) \quad \mathbb{M}_{2}=\left(\begin{array}{cc}
L & M \\
M & N
\end{array}\right)
$$

Principal curvatures $k_{\max }$ and $k_{\text {min }}$ are the eigenvalues of the matrix $\mathbb{M}_{1}^{-1} \mathbb{M}_{2}$ by definition [31]. The product of eigenvalues is equal to the determinant of the matrix and we get the gaussian curvature in Equation 3.15 in terms of the first and second fundamental form model parameters.

$$
K=\operatorname{det}\left(\mathbb{M}_{1}^{-1} \mathbb{M}_{2}\right)=\frac{L N-M^{2}}{E G-F^{2}}
$$

Trace of the matrix $\mathbb{M}_{1}^{-1} \mathbb{M}_{2}$ is the sum of eigenvalues, therefore mean curvature is obtained as in Equation 3.16 in terms of the first and second fundamental form model parameters.

$$
H=\frac{1}{2} \operatorname{trace}\left(\mathbb{M}_{1}^{-1} \mathbb{M}_{2}\right)=\frac{1}{2} \frac{L G-2 F M+N E}{E G-F^{2}}
$$

Principal curvatures can be recovered from $\mathrm{H}$ and $\mathrm{K}$ as in equations 3.17 and 3.18. It is straight forward to get shape index and curvedness values using Equations 3.3 and 3.4 .

$$
\begin{aligned}
& k_{\max }=H+\sqrt{H^{2}-K} \\
& k_{\text {min }}=H-\sqrt{H^{2}-K}
\end{aligned}
$$

Previously, we have fitted the 3D face data on 400x320 uniform grid having $0.5 \mathrm{~mm}$ resolution in X,Y plane interpolated/resampled Z values. All shape maps are calculated on this 400x320 uniform grid and therefore are also sized 400x320. We give 3D shape maps corresponding to SI, C, H and $\mathrm{K}$ for a sample face in Figure 3.3. 


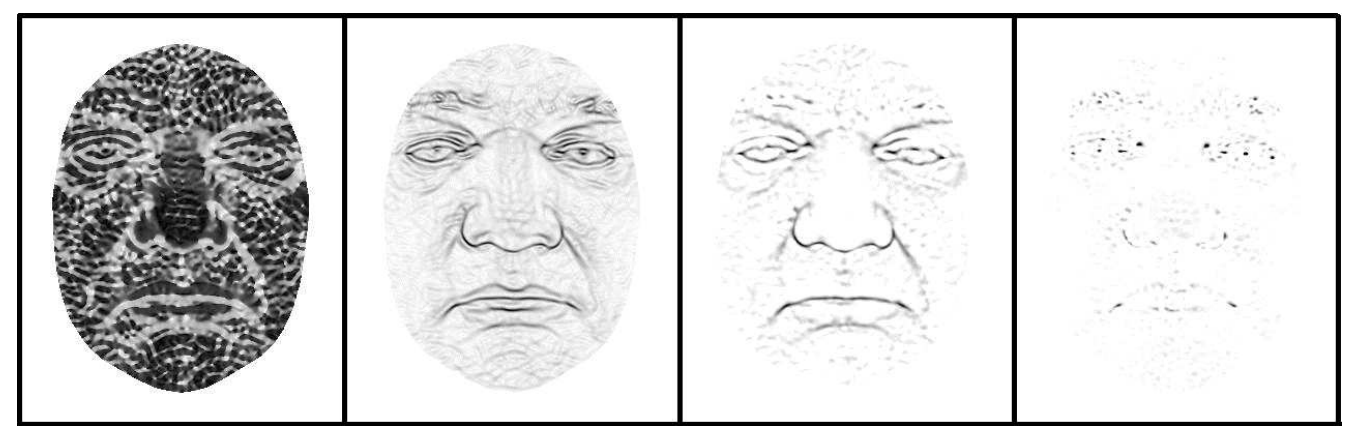

Figure 3.3: Sample shape maps ( SI, C, H and K maps)

\subsubsection{Uniformly Distributed Local Shape Descriptors for 3D Face Recog- nition}

For a typical classification problem, number of total dimensions to be used is limited by the curse of dimensionality. The curse of dimensionality is a term introduced by Bellman [6]. It stands for exponential increase in sparsity with increasing number of dimensions given a fixed amount of data points. Taking this fact into account, we select a moderate number of features to realize the recognition process. 457 feature locations on 400x320 grid is shown in Figure 3.4. The grid step size is 14 millimeters. Since the nose region is commonly reported to have discriminative information for $3 \mathrm{D}$ face recognition, the grid is designed to be more dense around the nose and the step size is reduced to 7 millimeters around the nose.

\subsubsection{D Feature Descriptors applied on 3D Shape Maps}

Following the calculation of the shape maps and deciding on the locations of the features, 2D feature descriptors can be applied on the shape maps easily. For 3D face recognition, we use two types of descriptors : SIFT and SURF. Here we should note three important points about the application of these descriptors in our method that differs from their conventional use in literature:

- Scale invariance is not required. Face recognition problem with data having 


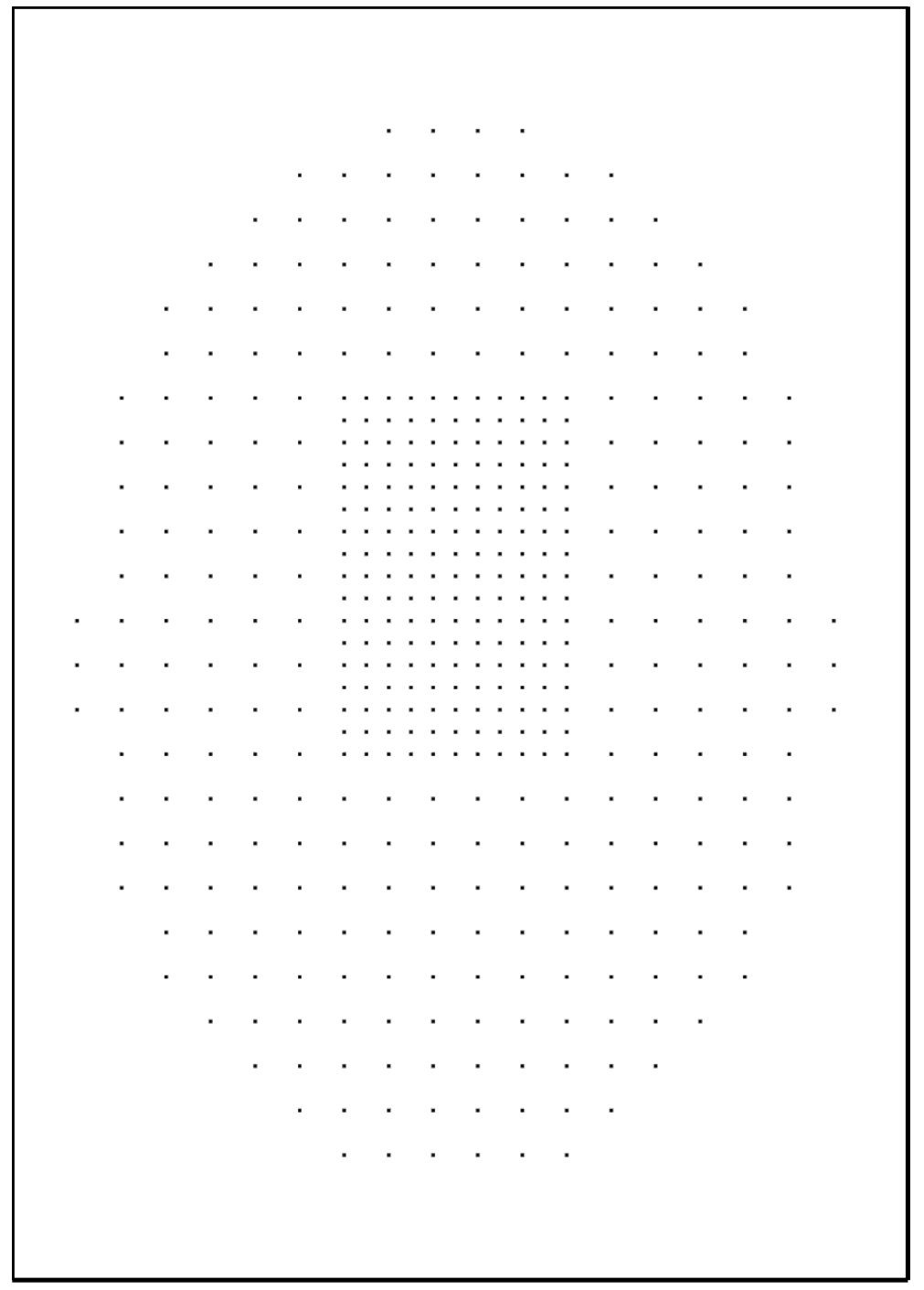

Figure 3.4: 457 nonuniformly distributed locations on 400x320 uniform grid 
metric $\mathrm{X}, \mathrm{Y}, \mathrm{Z}$ values does not require scale-invariance. The scale is fixed. In other words, a big face is big and a small one is small.

- Orientations of interest points are fixed. Once the face is registered globally, the orientations of interest points are almost fixed.

- Locations of interest points are fixed. Locations of interest points are predefined. Therefore, we do not use interest point detectors for this purpose.

To sum up, we generate descriptors at fixed scale and fixed orientation on 3D shape maps at 457 feature locations on the 400x320 uniform grid.

Various shape descriptors were calculated for a particular surface patch. We tried using the following SIFT [43] and SURF descriptors on different shape maps and reported their performances for 3D face recognition. Local feature description is one of the major problems of image vision and understanding. SIFT has been widely used in the last decade for local feature detection and description. SIFT is capable of detecting scale, translation and rotation invariant features in 2D images. SIFT keypoint detector identifies keypoints by searching maxima in scale-space domain, which is obtained by using difference of gaussians. The SIFT detector then assigns a local scale and local orientation to the image patch (keypoint) to be described. Keypoint descriptors are calculated on the local image patch whose in-plane rotation and scale are normalized with the scale and orientation values found in the keypoint detection step. Keypoint descriptors of SIFT are orientation histograms of the image gradient calculated by using the 16 pixels-by-16 pixels neighborhood of the keypoint. 16 pixels-by-16 pixels region is divided into 164 pixels-by-4 pixels regions. An orientation histogram with 8 angle bins is generated for each region. To sum up, 128 dimensional (16 regions-by- 8 orientation bins) descriptor is obtained for each keypoint.

Speeded up robust features (SURF) have local information including four constituents. These four constituents are as follows:

- Summation of magnitudes of Haar wavelet responses in the horizontal direction

- Summation of absolute values of Haar wavelet responses in the horizontal direc- 
tion

- Summation of magnitudes of Haar wavelet responses in the vertical direction

- Summation of absolute values of Haar wavelet responses in the vertical direction

These four constituents are calculated for 16 regions in the keypoint neighborhood, which gives 64 dimensional SURF descriptors for each keypoint. SURF descriptors are calculated with local orientations and local scales. All orientation angles are set to zero degrees for both SIFT and SURF descriptors. Our scale notation for SIFT descriptors refers to the length of one side of the local image patch (16 pixels-by-16 pixels) in millimeters. For SURF descriptors we indicate the scale by giving standard deviation of the smoothing gaussian function.

All features calculated at locations shown in Figure 3.4, and a sample descriptor set for four millimeter wide SIFT feature is given in Figure 3.5.

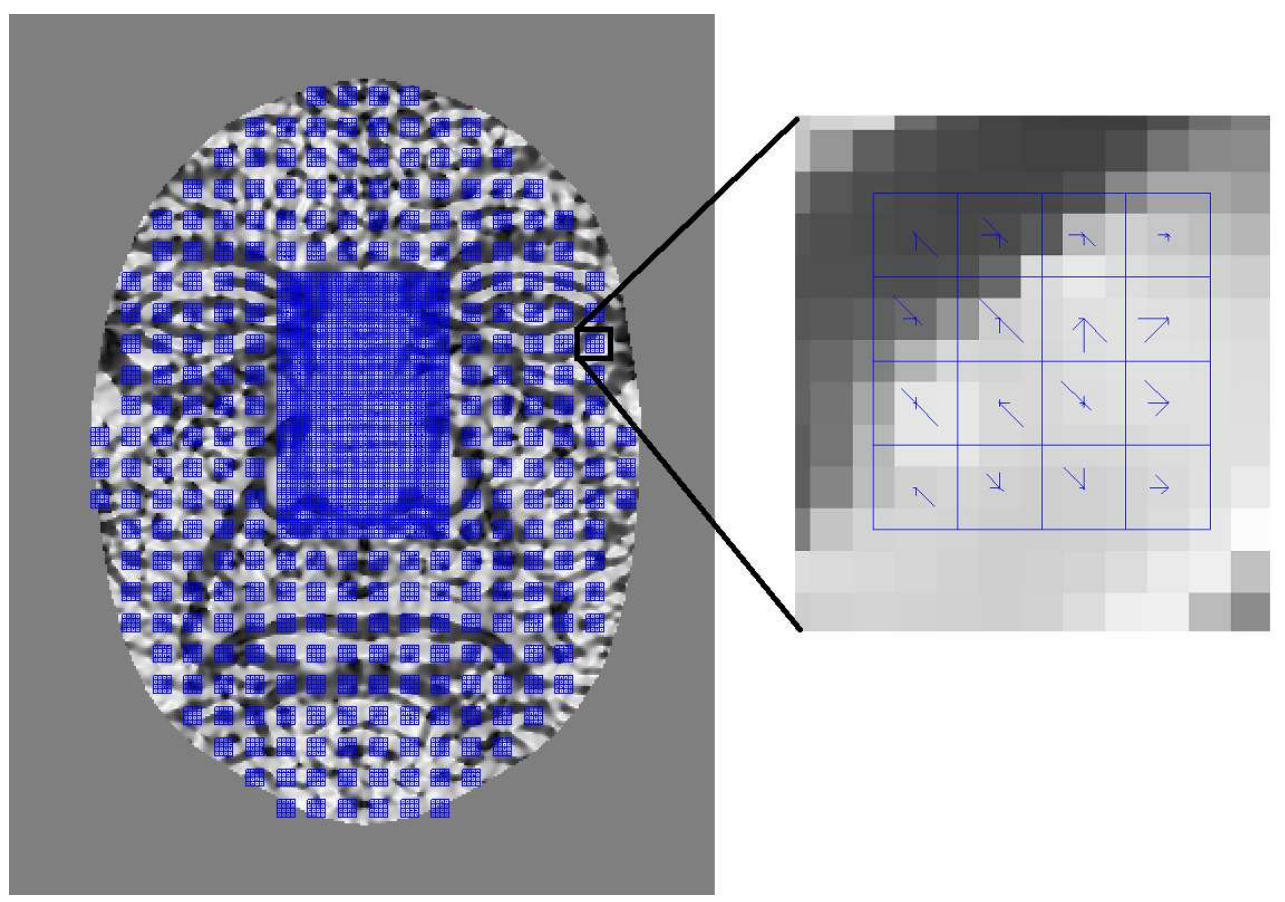

Figure 3.5: SIFT Descriptors calculated on shape index map at 457 uniformly distributed locations 


\subsection{Dimension Reduction}

Generating $d$ dimensional feature vectors $(d=128$ for the SIFT descriptor, $d=$ 64 for the SURF descriptor $)$ at $n$ nodes $(n=457)$ results in $n \times d$ dimensional descriptors for each face. It is essential to summarize the information stored in the $n \mathrm{x}$ $d$ dimensional total descriptor. Although $n \times d$ dimensional feature vector includes a lot of shape information, it is essential to extract subject-discriminative part of this information. At this stage, Fisher's linear discriminant analysis (LDA) can be used as the dimension reduction technique. LDA tries to find an optimal set of the discriminant projection vectors to map the original feature space onto a lower-dimensional feature space, by maximizing the Fisher criterion[20]:

$$
J_{F}(\mathbf{W})=\frac{\left|\mathbf{W}^{T} S_{b} \mathbf{W}\right|}{\left|\mathbf{W}^{T} S_{w} \mathbf{W}\right|}
$$

Where

$S_{b}:$ between - class scatter matrix

$S_{w}:$ within - class scatter matrix

$W$ : projection matrix

By maximizing the Fisher criterion, a special linear projection matrix is obtained, which minimizes within class variances and maximizes between class variances of the projected data.

\subsection{Classification}

After the calculation of the discriminative subspace for the training set, all the face data to be tested is projected onto this subspace. Classification is done by comparing target and query data by the help of a similarity metric (euclidean, cosine, etc). We use the cosine distance as the similarity metric since it outperformed the euclidean metric in our experiments. 


\subsection{Computational Complexity of the Proposed 3D Face Recognition System}

We consider each stage at Figure 3.1 for computational complexity evaluation. We will state the complexity values for preprocessing, differential shape map generation, descriptor calculation, subspace projection and classification(similarity measurement generation) respectively.

\subsubsection{Computational Complexity of the Preproccesing Stage}

Most time consuming part of the preprocessing stages is registration where we calculate symmetry map of the shape index image for every rotated line. The time complexity of the registration stage can be stated as in Equation 3.20.

$$
t c_{\text {preprocess }}=O(n i) \text {; }
$$

where

$t c_{\text {preprocess }}:$ time complexity

$n$ : the number of data samples

$i$ : the number of the rotation iterations

\subsubsection{Computational Complexity of the Differential Map Generation Stage}

The time complexity of the differential shape map generation stage can be stated as in Equation 3.21.

$$
t c_{d i f f}=O(n) ;
$$

where 
$n$ : the number of data samples

\subsubsection{Computational Complexity of the Descriptor Calculation Stage}

The time complexity of the descriptor calculation stage can be stated as in Equation 3.22 .

$$
t c_{\text {descriptor }}=O(s n)
$$

where

$s$ : the number of scale - space - location combinations

$n$ : the number of data samples

\subsubsection{Computational Complexity of the LDA Stage (TRAINING)}

We have used the LDA implementation of Cai [12]. He displays the time complexity of his implementation as in Equation 3.23

$$
t c_{L D A}=O\left(\operatorname{m.n} \cdot \min (n, m)+(\min (n, m))^{3}\right)
$$

where

$n$ : the number of data samples

$m$ : the number of data features

\subsubsection{Computational Complexity of the LDA Stage (TESTING)}

For the testing case, time complexity of the LDA stage is equal to the that of matrix multiplication as in Equation 3.24. 


$$
t c_{\text {projection }}=O(n * D * d)
$$

where

$n$ : the number of data samples

$D$ : the initial number of dimension of data features

$d$ : the reduced number of dimension of data features

\subsubsection{Computational Complexity of the Classification Stage}

For the classification stage, time complexity is as in Equation 3.25

$$
t c_{\text {classification }}=O(n * g)
$$

where

$n$ : the number of data samples in the probe set

$g$ : the number of data samples in the gallery set

\subsubsection{Computational Complexity of the Training}

Time complexity of the training stage can be calculated as the summation of the time complexities of the required stages for training. Resultant time complexity of the training is given in Equation 3.26. The equation can be expressed as in Equation 3.27

$$
t c_{\text {training }}=t c_{\text {preprocess }}+t c_{\text {diff }}+t c_{\text {descriptor }}+t c_{L D A}
$$




$$
t c_{\text {training }}=O(n i)+O(n)+O(s n)+O\left(\operatorname{m} . n \cdot \min (n, m)+(\min (n, m))^{3}\right)
$$

\subsubsection{Computational Complexity of the Testing}

Time complexity of the testing stage can be calculated as the summation of the time complexities of the required stages for testing. Resultant time complexity of the testing the all probe images is given in Equation 3.28. $t c_{\text {classification }}$ is the dominating expression in Equation 3.28. The equation can also be expressed as in Equation 3.29.

$$
t c_{\text {testing }}=t c_{\text {preprocess }}+t c_{\text {diff }}+t c_{\text {descriptor }}+t c_{\text {projection }}+t c_{\text {classification }}
$$

$$
t c_{\text {testing }}=O(n i)+O(n)+O(s n)+O(n * D * d)+O(n * g)
$$




\section{CHAPTER 4}

\section{GENERIC MODEL SUPPORTED 3D FACE RECOGNITION}

\subsection{Introduction}

3D generic face models have been widely used in practical problems such as facial animation, face tracking, facial expression recognition, and face recognition. All human faces share a similar topological structure. Taking this trivial fact into account, many face recognition studies use generic face models prior to recognition process. Faces are usually modeled as graphs/meshes with nodes positioned at fiducial points such as eye corners, nose tip etc. Therefore, using generic face models gives the researchers the opportunity of finding conjugate pairs of fudicial points at the faces to be analyzed. This chapter will present a generic model supported 3D face recognition method. The presented generic model generation approach is based on radial basis fitting. Radial basis function fitting needs a limited number of node points to be initiated. Fiducial points on the face can be used as initial radial basis function nodes, which can be located either manually or automatically. We have marked them manually on the FRGC database [38]. We begin with introducing facial annotation process and then explain the method used for generic model fitting.

\subsection{Annotated Face Database}

3D FRGC database is provided by University of Notre Dame. The outline of the FRGC database is given in Section 5.1. Top and side views of a sample 3D face scan are given in Figure 4.1 and Figure 4.2 respectively. A 2D image accompanying the 3D 
face scan is displayed in Figure 4.3.

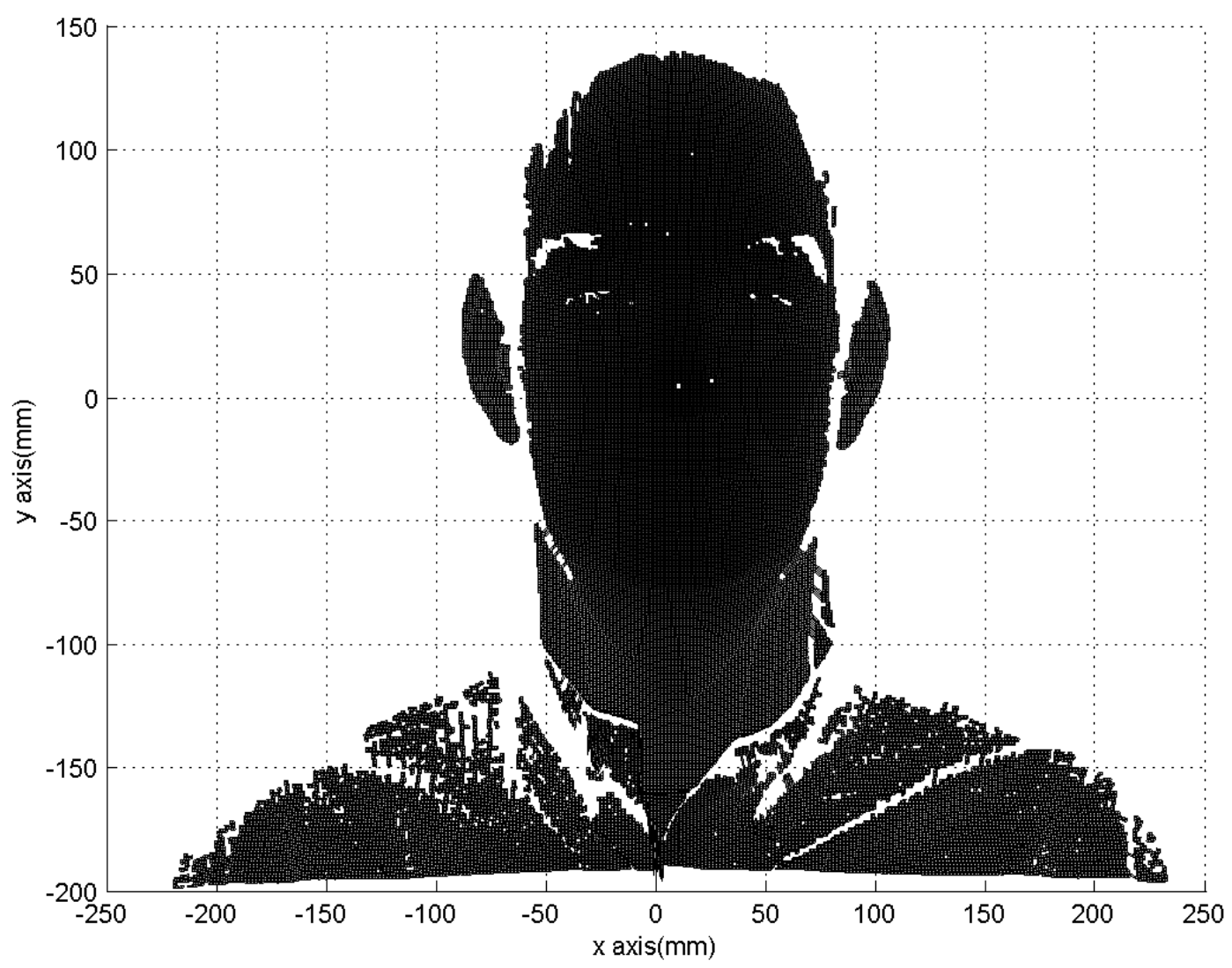

Figure 4.1: Sample 3D Face Scan in FRGC Database (front view)

\subsection{Manual Annotation of Facial Feature Points}

\subsubsection{Annotated Facial Feature Points}

The node points are the initial input of the generic face model used in this study, hence prior to generic face model generation the face database to be studied should be marked. We selected a subset of generic model nodes to be marked. In the following section, we give the set of facial animation points with their locations and codes. Once the facial feature points are selected, annotation can be completed. The interactive tool developed for this purpose and the marking process are also explained in the 


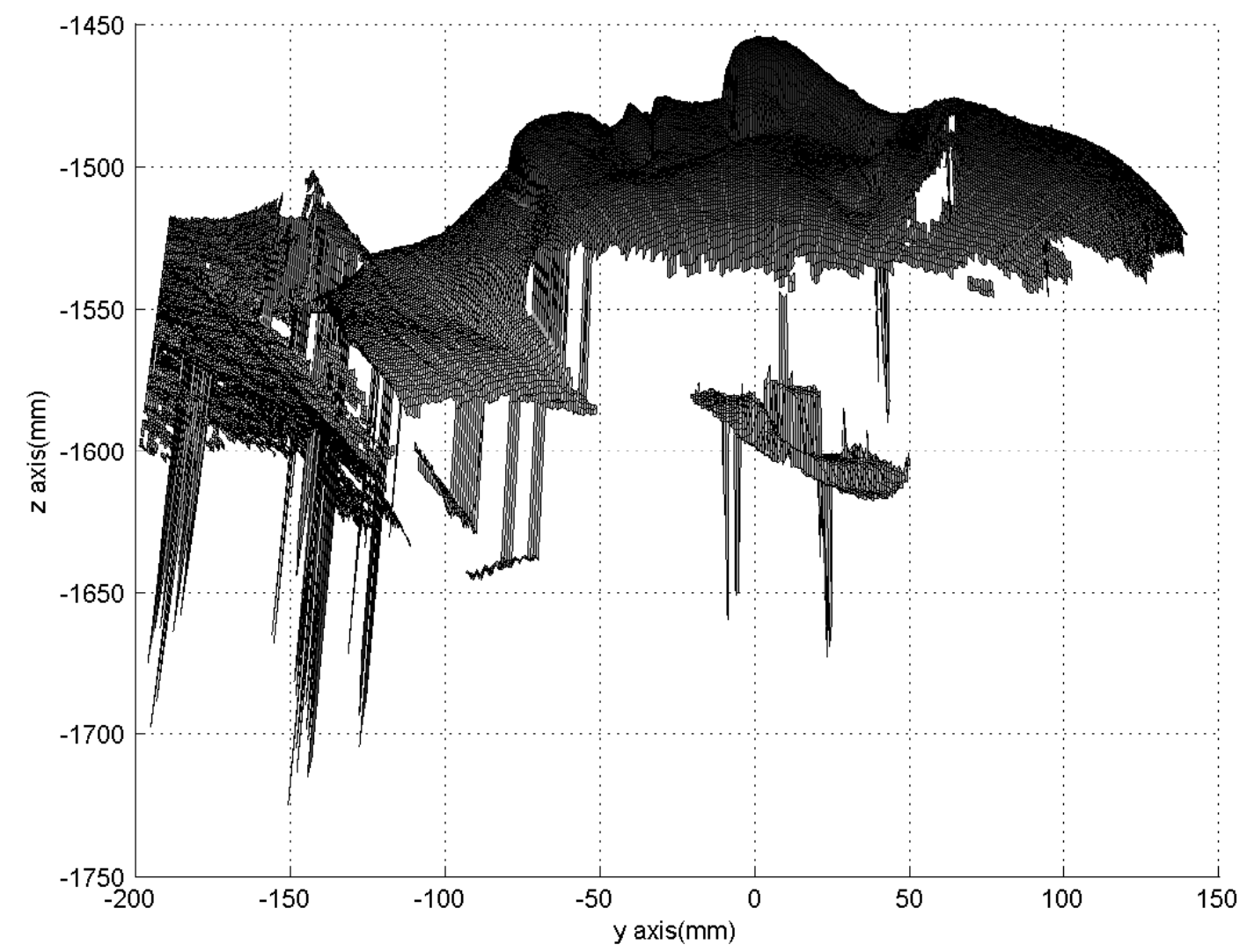

Figure 4.2: Sample 3D Face Scan in FRGC Database (side view) 


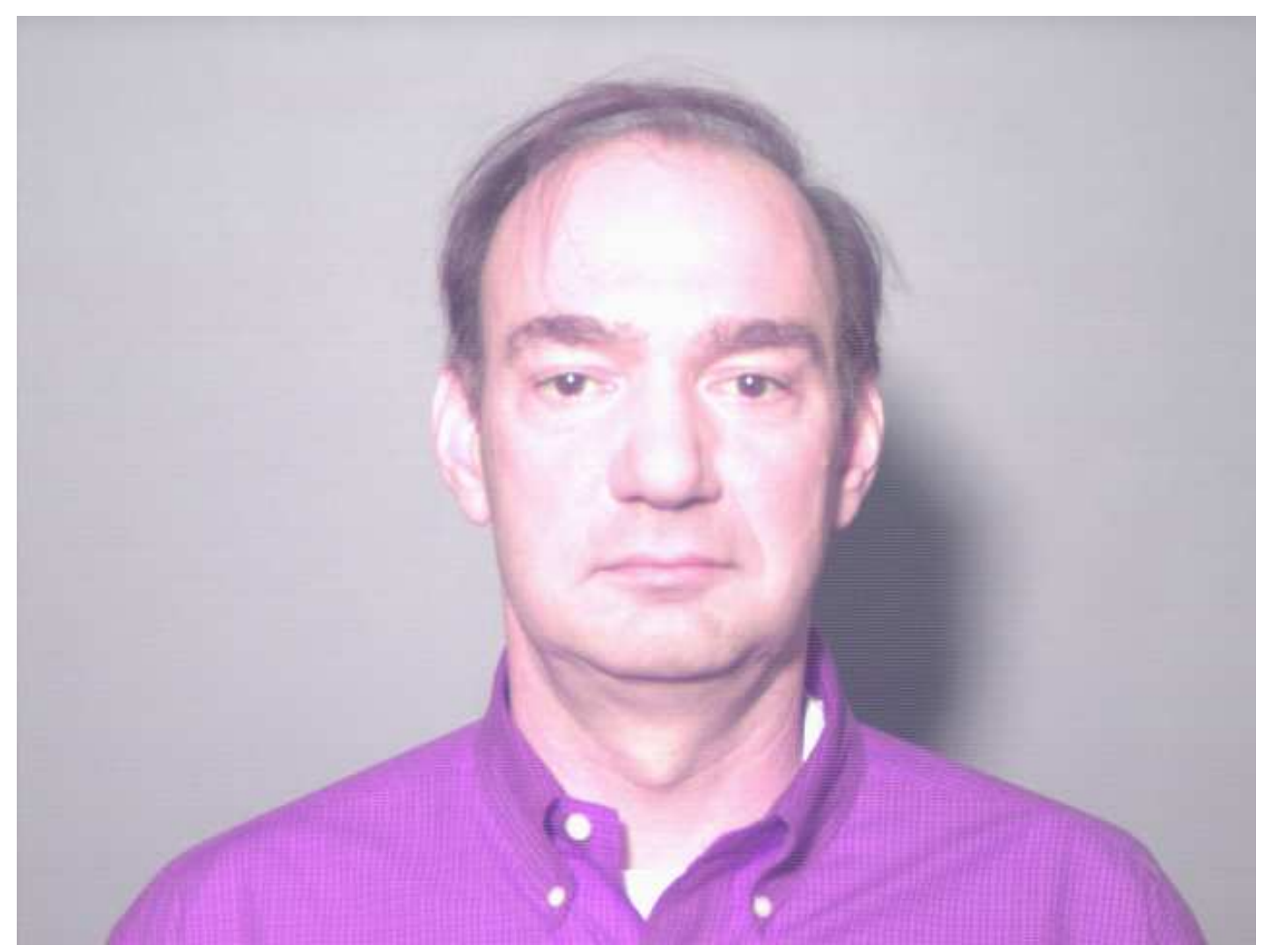

Figure 4.3: Sample 2D Face Image accompanying the 3D scan in FRGC Database 
following section.

Facial feature points to be annotated are selected from the facial animation points given in International Standard ISO/IEC 14496-2 [27]. 50 facial feature points, which are manually marked, and their codes and explanations in [27] are given in Table 4.1, Table 4.2 and Figure 4.4.

Table 4.1: Manually Marked Facial Feature Points

\begin{tabular}{lll}
\hline$\#$ & ISO 14469-2 code & Description \\
\hline 1 & 11.2 & Right border between hair and forehead \\
\hline 2 & 11.1 & Middle border between hair and forehead \\
\hline 3 & 11.3 & Left border between hair and forehead \\
\hline 4 & 4.6 & Right corner of right eyebrow \\
\hline 5 & 4.4 & Uppermost point of the right eyebrow \\
\hline 6 & 4.2 & Left corner of right eyebrow \\
\hline 7 & 4.1 & Right corner of left eyebrow \\
\hline 8 & 4.3 & Uppermost point of the left eyebrow \\
\hline 9 & 4.5 & Left corner of left eyebrow \\
\hline 10 & 3.12 & Right corner of right eye \\
\hline 11 & 3.14 & Center of upper outer right eyelid \\
\hline 12 & 3.10 & Center of lower outer right eyelid \\
\hline 13 & 3.8 & Left corner of right eye \\
\hline 14 & 9.6 & Right upper edge of nose bone \\
\hline 15 & 9.7 & Left upper edge of nose bone \\
\hline 16 & 3.11 & Right corner of left eye \\
\hline 17 & 3.13 & Center of upper outer left eyelid \\
\hline 18 & 3.9 & Center of lower outer left eyelid \\
\hline 19 & 3.7 & Left corner of left eye \\
\hline 20 & 5.4 & Right cheek bone \\
\hline 21 & 9.12 & Nose bump \\
\hline 22 & 5.3 & Left cheek bone \\
\hline 23 & 9.2 & Right nostril border \\
\hline 24 & 9.3 & Nose tip \\
\hline & & Left nostril border \\
\hline
\end{tabular}

\subsubsection{Interactive Tool for Annotation Facial Feature Points}

Annotation of facial feature points can be realized in two different ways. The first approach is directly annotating the 3D face data. This interface should be capable of displaying and marking a 3D point cloud. The second way of annotating 3D face data is marking $2 \mathrm{D}$ images associated with the $3 \mathrm{D}$ face data. $2 \mathrm{D}$ images are perceived and 

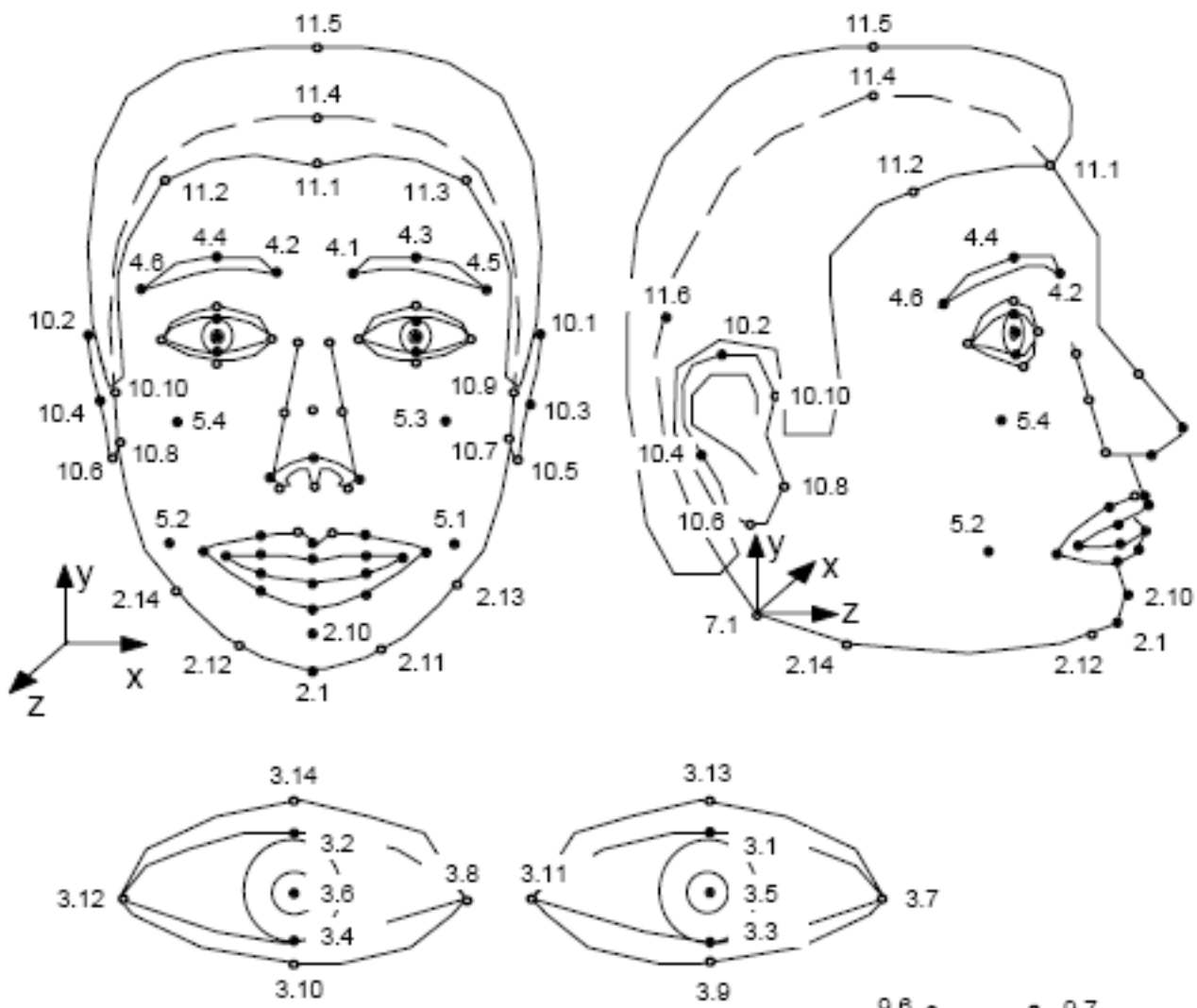

Right eye

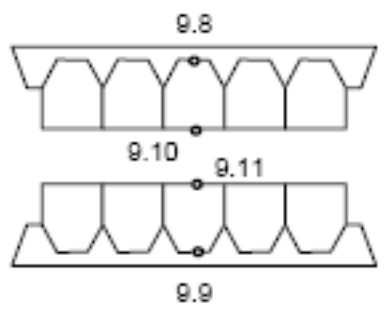

Teeth

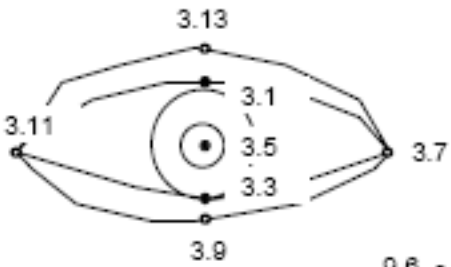

Left eye
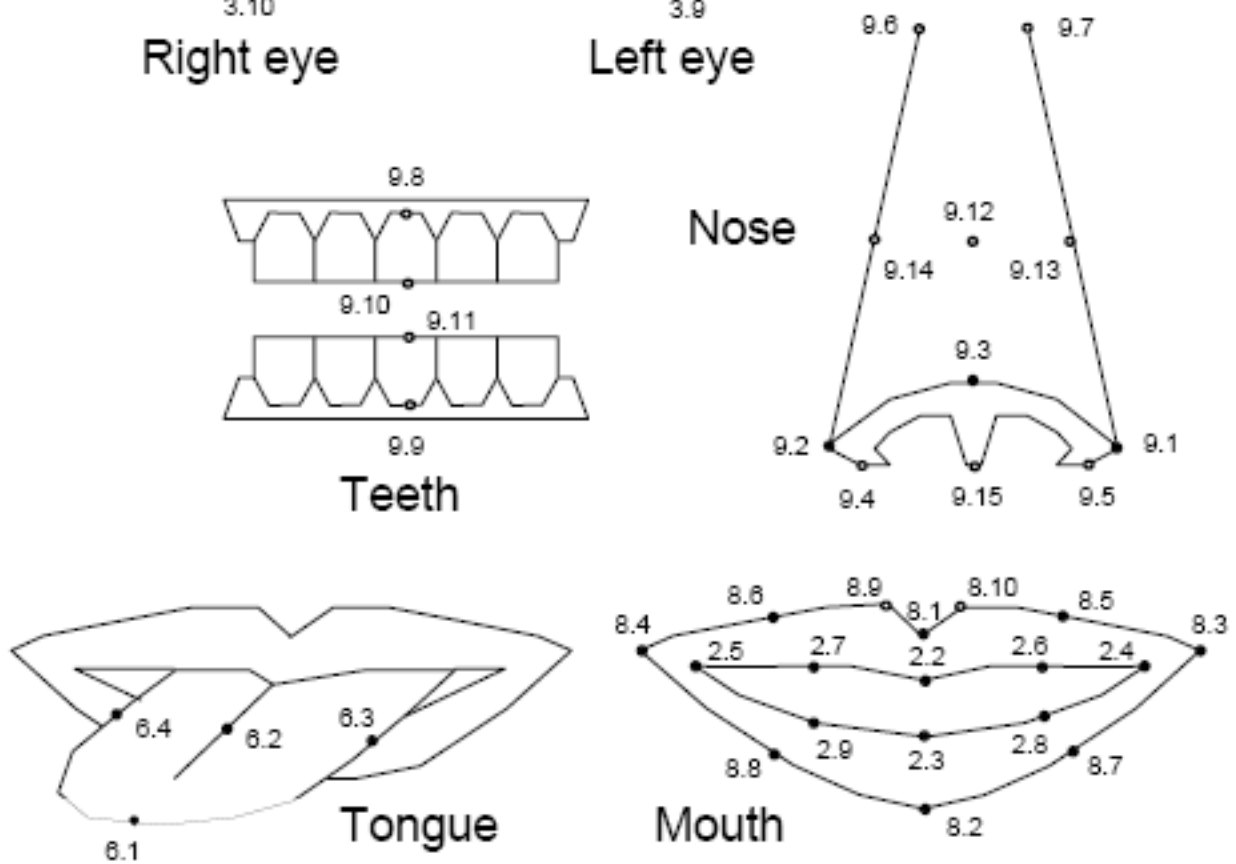

Figure 4.4: Facial Animation Points ISO 14496 Codes [27] 
Table 4.2: Manually Marked Facial Feature Points (continued)

\begin{tabular}{lll}
\hline$\#$ & ISO 14469-2 code & Description \\
\hline 26 & 9.4 & Bottom right edge of nose \\
\hline 27 & 9.15 & Bottom middle edge of nose \\
\hline 28 & 9.5 & Bottom left edge of nose \\
\hline 29 & 5.2 & Center of right cheek \\
\hline 30 & 8.4 & Right corner of outer lip contour \\
\hline 31 & 8.6 & Midpoint between 8.4 and 8.1 in outer upper lip contour \\
\hline 32 & 8.9 & Right hiph point of Cupid's bow \\
\hline 33 & 8.1 & Middle point of outer upper lip contour \\
\hline 34 & 8.10 & Left hiph point of Cupid's bow \\
\hline 35 & 8.5 & Midpoint between 8.3 and 8.1 in outer upper lip contour \\
\hline 36 & 8.3 & Left corner of outer lip contour \\
\hline 37 & 5.1 & Center of left cheek \\
\hline 38 & 2.5 & Right corner of inner lip contour \\
\hline 39 & 2.2 & Middle point of inner upper lip contour \\
\hline 40 & 2.3 & Middle point of inner lower lip contour \\
\hline 41 & 2.4 & Left corner of inner lip contour \\
\hline 42 & 8.8 & Midpoint between 8.4 and 8.2 in outer lower lip contour \\
\hline 43 & 8.2 & Middle point of outer lower lip contour \\
\hline 44 & 8.7 & Midpoint between 8.3 and 8.2 in outer lower lip contour \\
\hline 45 & 2.14 & Right corner of jaw bone \\
\hline 46 & 2.12 & Chin right corner \\
\hline 47 & 2.10 & Chin boss \\
\hline 48 & 2.1 & Bottom of the chin \\
\hline 49 & 2.11 & Chin left corner \\
\hline 50 & 2.13 & Left corner of jaw bone \\
\hline & &
\end{tabular}


annotated easier than their 3D counterparts. In Figure 4.5, the interactive tool developed in METU Computer Vision and Intelligent Systems Research Laboratory for face annotation is shown. 2D images are not directly used in the annotation process. Pixels of the 2D face image, which correspond to invalid 3D data are displayed as dark blue. During the annotation process, special care is taken not to mark invalid pixels (i.e dark blue pixels). Consequently, almost all of the pixels that have been marked have valid 3D data.

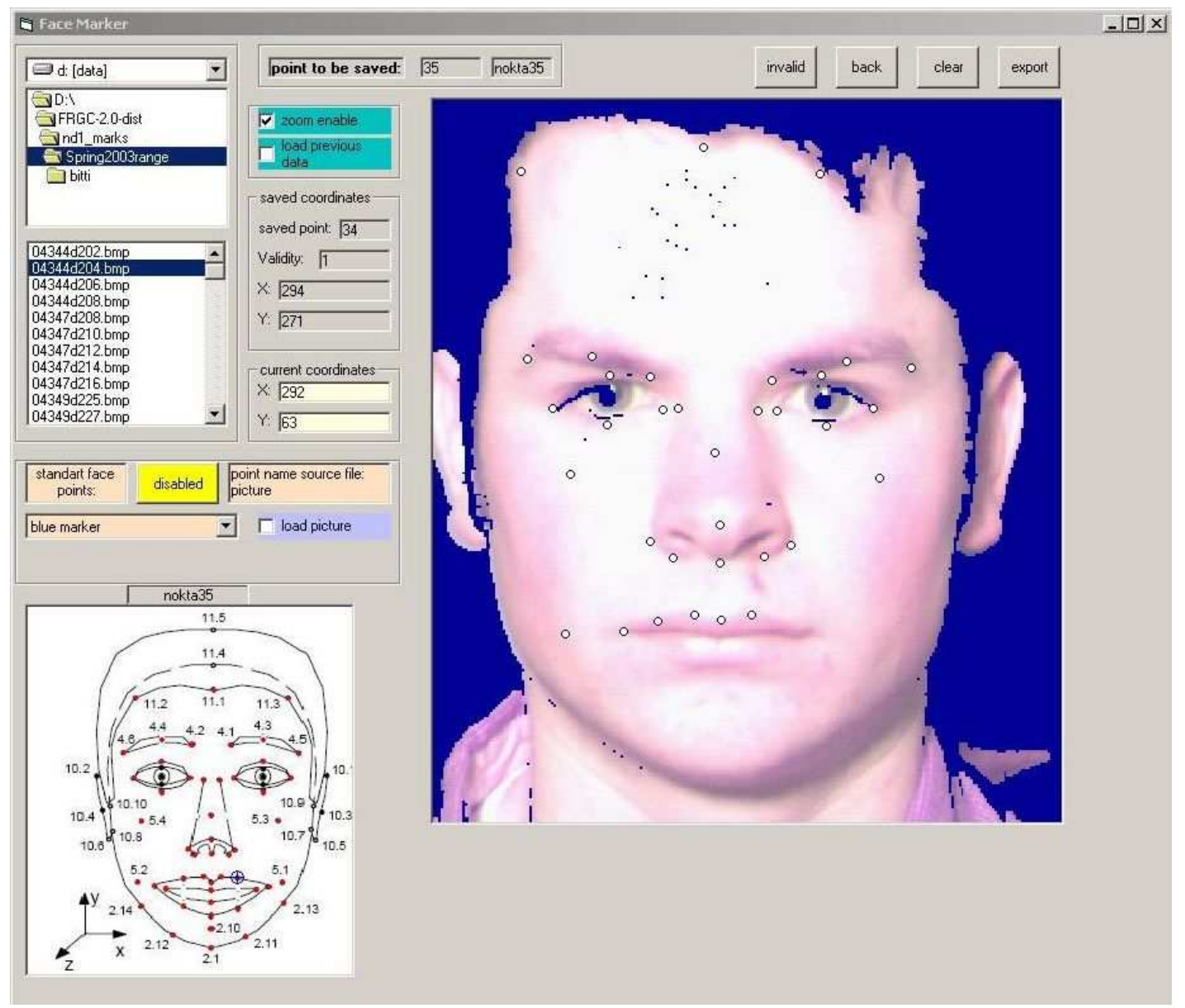

Figure 4.5: Interactive Tool for Face Annotation

FRGC v1.0 database [38] has 943 and FRGC v2.0 database has 4007 3D face recordings. This makes a total of 4950 3D face images. In our annotation process, 29 images were unmarked due to the inconsistency between 2D and 3D images. A sample in- 
consistent image is shown in Figure 4.6. Remaining 4921 face recordings were all annotated. A sample annotated image is shown in Figure 4.7

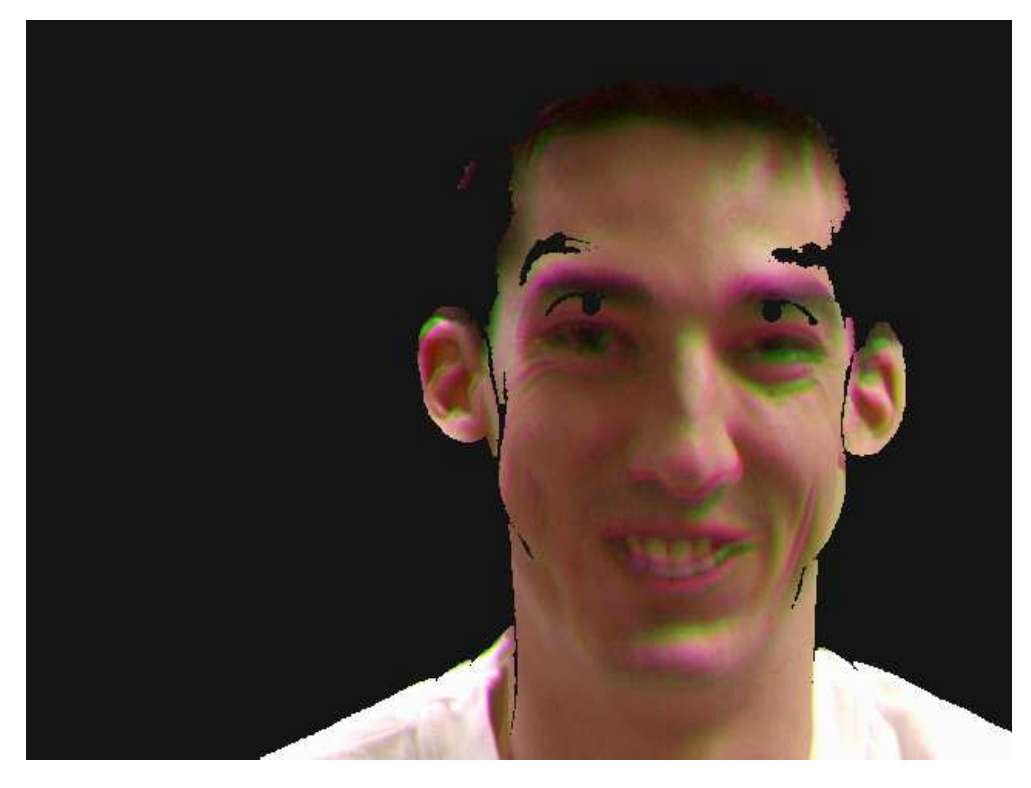

Figure 4.6: Face recording having inconsistency between 2D and 3D images

\subsubsection{Updating Facial Feature Points}

Manual marking of many facial feature points are quite consistent among different records of the same subjects, however some facial feature points have still ambiguous coordinates. These facial feature points' coordinates are updated according to the recommendations given in ISO/IEC 14496-2 [27]. Updated facial feature points and the ways they are updated are listed below:

- Center of left cheek (5.1) The y-coordinate is set to the y-coordinate of left corner of outer lip contour (8.3) and the closest data point to the new coordinate triple is assigned as the center of left the cheek.

- Center of right cheek (5.2) The y-coordinate is set to the y-coordinate of the right corner of the outer lip contour (8.4) and the closest data point to the new coordinate triple is assigned as the center of the right cheek. 


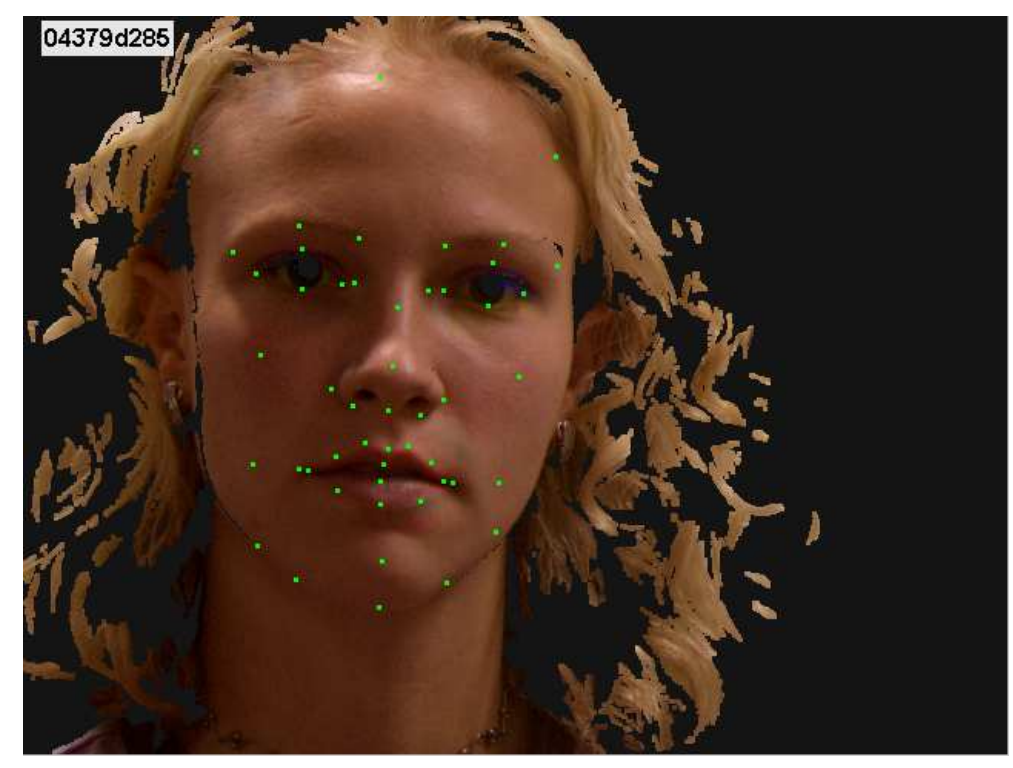

Figure 4.7: Sample annotated face recording

- Nose bump(9.12) The y-coordinate is set to the mean of y-coordinates of the right upper edge of the nose bone (9.6) and the nose tip (9.3). The closest data point to the new coordinate triple is assigned as the center of the right cheek.

- Right cheek bone (5.4) X-coordinate is set to the mean of $\mathrm{x}$-coordinates of the center of the pupil of the right eye (3.6) and the right corner of the right eye (3.12). The y-coordinate is set to the mean of y-coordinates of the bottom middle edge of the nose (9.15) and the nose bump (9.12). The closest data point to the new coordinate triple is assigned as the left cheek bone. Since the center of the pupil of right eye (3.6) is not among the marked points, its x-coordinate is estimated as the mean of the $\mathrm{x}$-coordinates of the center of the upper outer right eyelid (3.14) and the center of the lower outer right eyelid (3.10).

- Left cheek bone (5.3) The x-coordinate is set to the mean of the x-coordinates of the center of the pupil of the left eye (3.5) and the left corner of the left eye (3.7). The $y$-coordinate is set to the mean of $y$-coordinates of the bottom middle edge of the nose (9.15) and the nose bump (9.12). The closest data point to the new coordinate triple is assigned as the left cheek bone. Since the center of the pupil of the left eye (3.5) is not among the marked points, its x-coordinate is 
estimated as the mean of the $\mathrm{x}$-coordinates of the center of the upper outer left eyelid(3.13) and the center of the lower outer left eyelid (3.9).

\subsection{Preprocessing of 3D Face Data}

Preprocessing stages are smoothing, registration, uniform resampling and cropping. These stages are explained in Section 3.2. Exactly the same procedure is applied for generic model supported 3D face recognition.

\subsection{Generic Model Fitting}

\subsubsection{Generic Face Model}

Generic face model fitting is frequently used in face recognition studies to a make common frame for probe and gallery images. This study also employs a generic face model [1]. Face model used in the study consists of 549 vertices, 488 quadrilaterals and 38 triangles. Complete set of vertices, triangles and quadrilaterals are given at Appendix A in Matlab syntax. Front and side views of the generic face model are shown in Figure 4.8 and Figure 4.9 respectively.

\subsubsection{Radial Basis Function Fitting}

After all rigid transformations are removed, a nonlinear deformation step is essential for face model fitting. Facial feature points are used at this step to establish a relation between the data and the model. The face and the data can be represented by facial feature points (vertices) as shown in equations 4.1 and 4.2 .

$$
\begin{array}{r}
\mathbb{F}_{i}=\left\{\overrightarrow{u_{i, 1}}, \overrightarrow{u_{i, 2}}, \overrightarrow{u_{i, 3}}, \ldots \overrightarrow{u_{i, n}}\right\} \\
\mathbb{G}=\left\{\overrightarrow{v_{1}}, \overrightarrow{v_{2}}, \overrightarrow{v_{3}}, \ldots \overrightarrow{v_{n}}\right\}
\end{array}
$$

where 


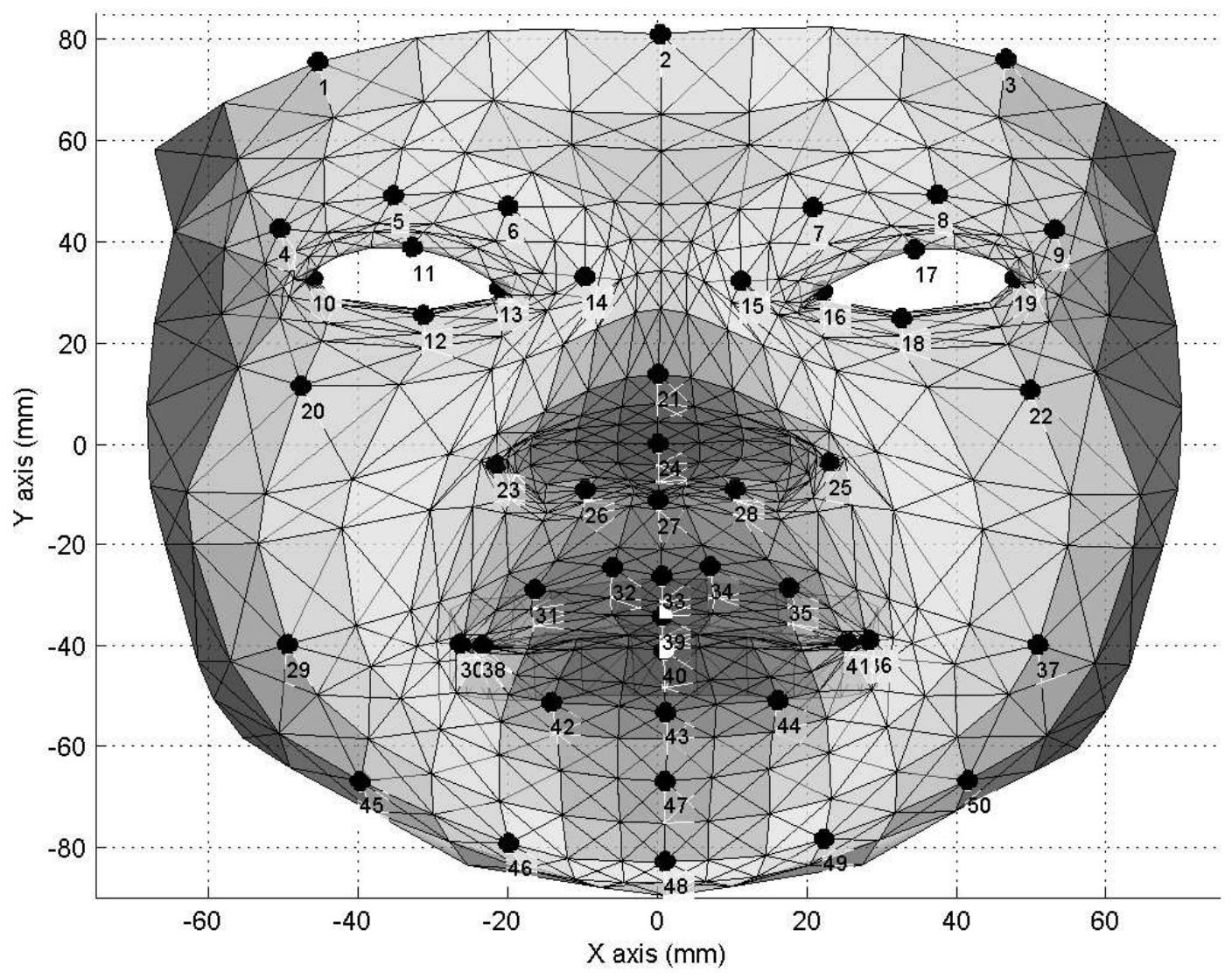

Figure 4.8: Generic Face Model (Front View) 


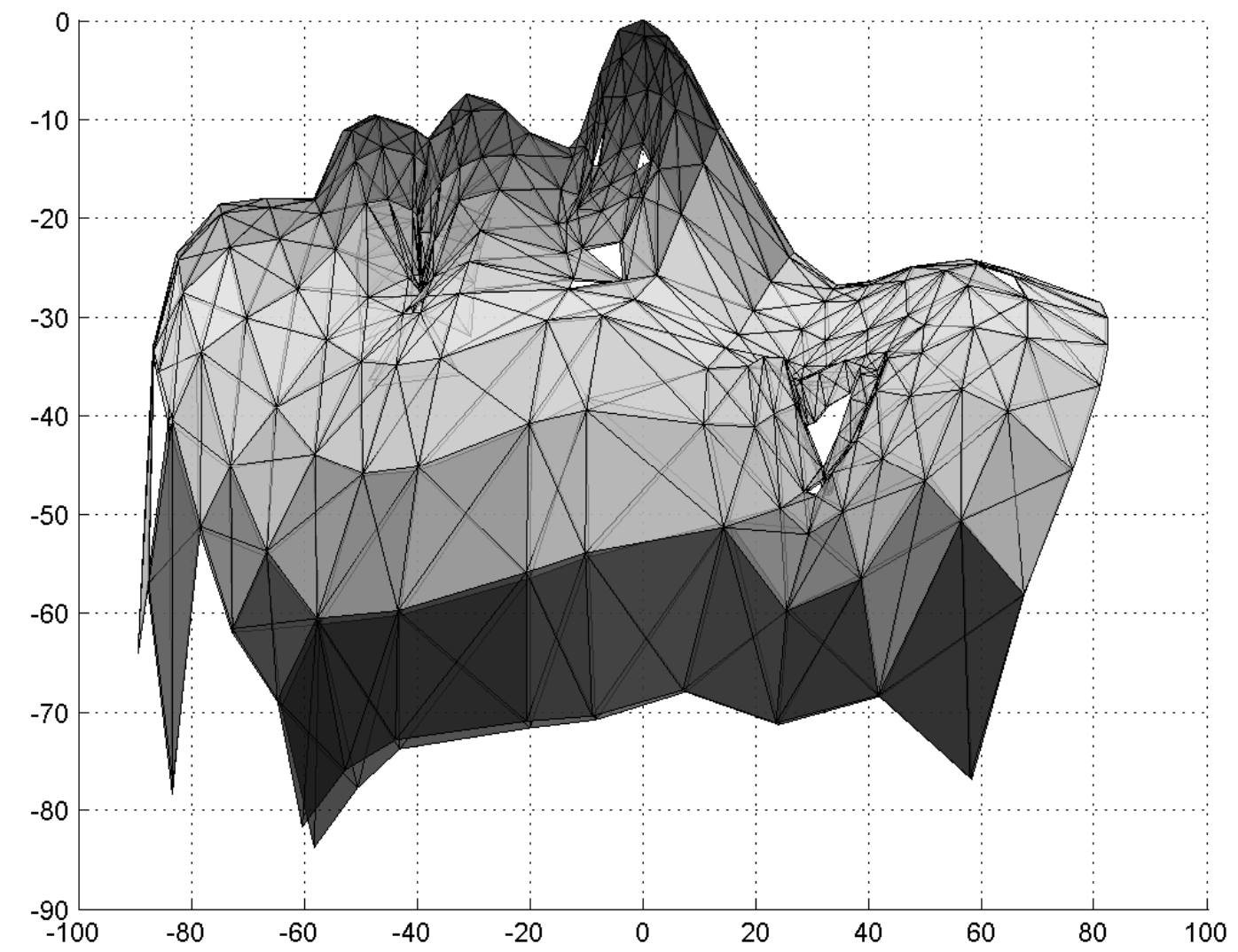

Figure 4.9: Generic Face Model (Side View) 
$\mathbb{F}_{i}:$ n element set representing $i^{\text {th }}$ face data

$\mathbb{G}:$ : element set representing face model

$u_{i, k}: 3 D$ coordinates of $k^{\text {th }}$ point of $i^{\text {th }}$ face data

$v_{k}: 3 D$ coordinates of $k^{\text {th }}$ point of face model

Then the deformation vector at the $k^{t h}$ vertex (facial feature point) can be defined as:

$$
\overrightarrow{d_{i, k}}=\overrightarrow{u_{i, k}}-\overrightarrow{v_{k}}
$$

At this point, model fitting problem is reduced to finding a global function, which is modeling all deformation vectors. Radial basis functions are commonly used for this purpose [49]. Radial basis function based on the global model can be formulated as in equation 4.4 .

$$
f(x)=\sum_{j=1}^{n} w_{j} \quad \phi\left(\left\|x-v_{j}\right\|\right)
$$

where

$f(x) \quad$ : the interpolating function

$w_{j} \in \mathbb{R}^{3}$ : interpolation weights

$\phi \quad$ : radial basis function

$\|\cdot\|$ : euclidean norm

Various basis functions are reported in literature. In [49], multi-quadric function is reported to have a good surface fitting performance. Multi-quadric function; $\phi(r)=$ $\sqrt{r^{2}+1}$ is used in this study. Interpolation weights of the radial basis function are found by solving the set of linear equations given in equation 4.5

$$
\overrightarrow{d_{i, j}}=f\left(v_{j}\right) \quad \text { for } \quad j=1,2, \ldots n
$$




\subsubsection{Local Deformation}

Vertices on the generic face model, which correspond to facial feature points, are wellfitted by radial basis function interpolation. The other vertices are also deformed by radial basis function interpolation, however they are not embedded in the face data. A final local deformation is performed on non-facial feature vertices to find their matches in the $3 \mathrm{D}$ face data. Local deformation is done by mapping the generic face vertices to the closest point in face data. For a sample face, deformation vectors which resulted from radial basis function fitting and local deformation are shown in Figure 4.10 and Figure 4.11 .

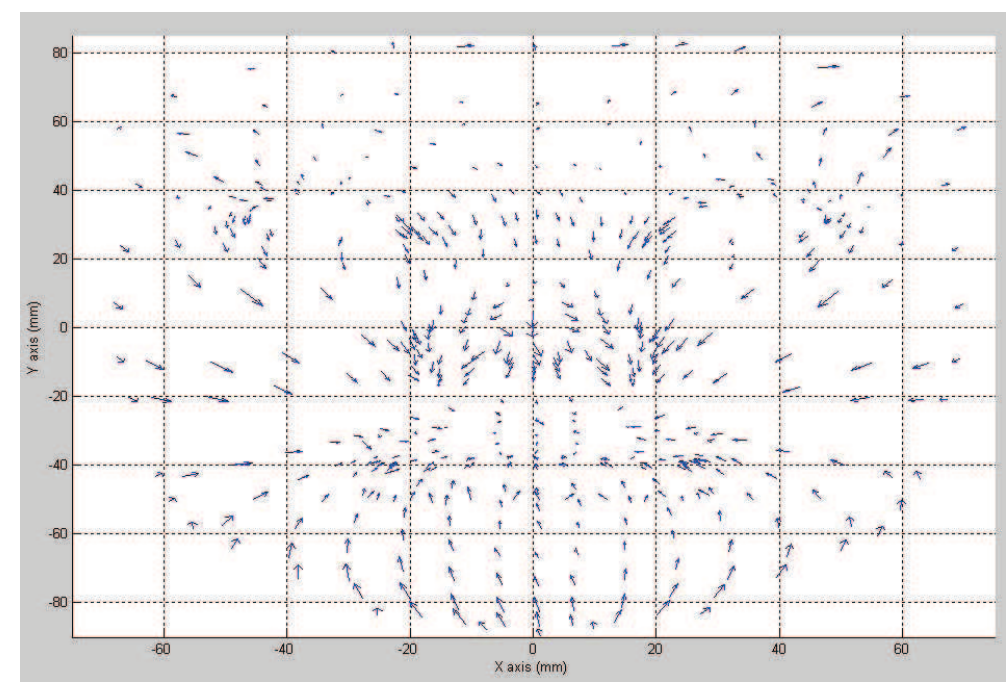

Figure 4.10: Deformation Vectors (Front View)

\subsubsection{Fine Registration and Resampling}

Final orientation correction is made on 3D face data after generic model fitting. Orientation correction is achieved by positioning the symmetry axis of shape index map as applied in the work of Alyuz and her friends [3]. Registration is completed by positioning the nose-tip at the origin. Registered 3D face image is interpolated and resampled at a uniform grid of $0.5 \mathrm{~mm}$ resolution both in $\mathrm{X}$ and Y directions. Uniform grid has a height of 400 pixels and a width of 320 pixels 


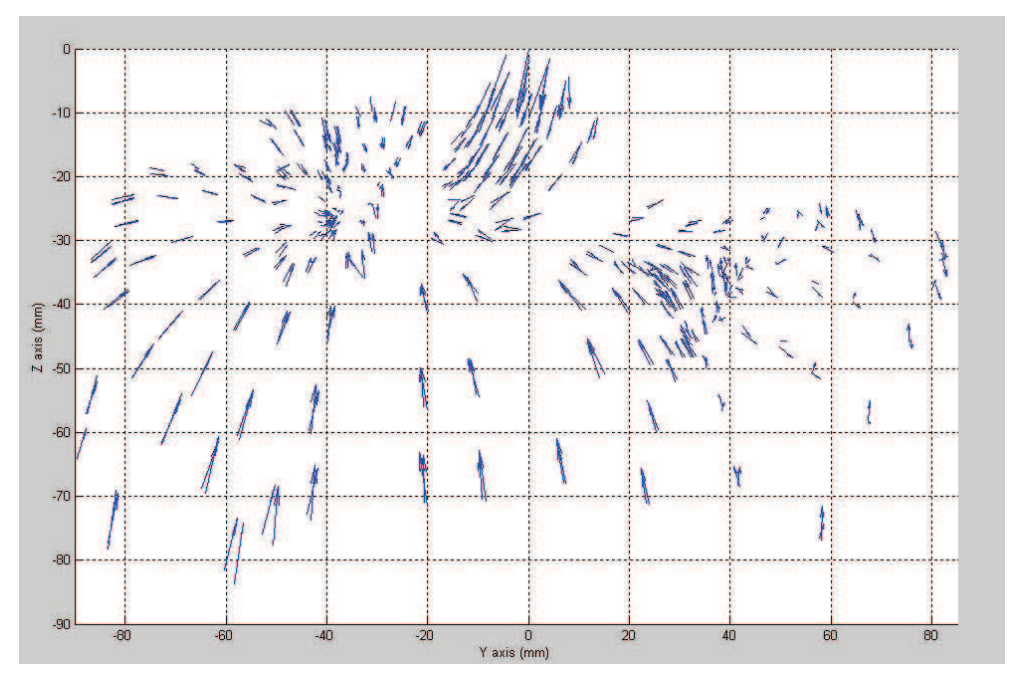

Figure 4.11: Deformation Vectors (Side View)

$\left(y_{\min }=-99.5, y_{\max }=100, x_{\min }=-79.5, x_{\max }=80\right)$. We should also note that annotated facial feature locations and all generic face model node locations are also updated accordingly and have their new locations in 400x320 uniform grid.

\subsection{Overview of the proposed generic model supported 3D face recog- nition system}

In figure 4.12, the block diagram of the proposed generic model supported 3D face recognition system is given. Following the acquisition of the $3 \mathrm{D}$ face data with a face scanner, 3D face data is registered and its spikes and holes are removed by smoothing. Preprocessing is finalized with uniform resampling and cropping. Preprocessed 3D face data are for calculation of differential shape parameters (shape index, curvedness, mean and gaussian curvature). SIFT and SURF operators are applied on the differential shape maps at the locations of the nodes of the deformed generic mesh. SIFT/SURF descriptors are then fed into dimension reduction stage and high-dimensional feature vector is projected onto a low-dimensional subspace. Identification/verification of the subjects are made in this low dimensional subspace. 

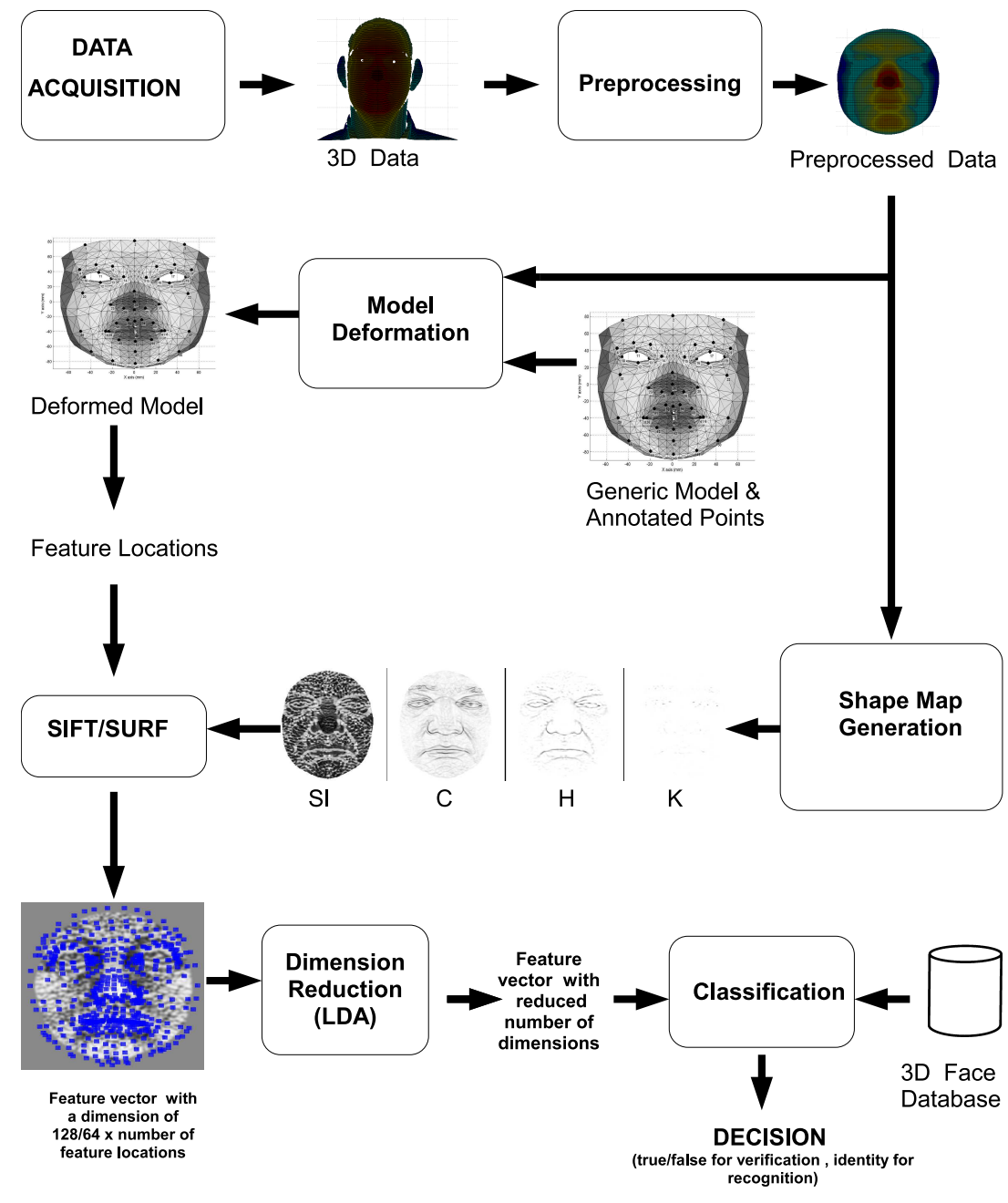

Figure 4.12: Block diagram of the proposed generic model supported 3D face recognition system 


\subsection{Local Shape Descriptors for Generic Model Supported 3D Face Recognition}

We have presented the annotation carried on the 3D face data and the radial basis function fitting for generic face modeling. Therefore, for every face in the $3 \mathrm{D}$ recognition process, we have the same set of generic 3D nodes as candidates for locations of local shape descriptors. Fitted elastic model is ready to serve as a robust frame for feature extraction step of the proposed generic model supported 3D face recognition system. This section presents the calculation of the local shape descriptors for the proposed generic model supported face recognition method.

\subsection{Feature Generation}

Feature generation for generic model supported face recognition requires calculation differential shape maps and local shape descriptors. These stages have already explained at Section 3.3. Details of shape map calculation and generation of local shape descriptor can be found at Section 3.3. The main characteristic of the generic model supported 3D face recognition is the subject specific selection of feature locations. Our generic face model has 549 vertices. All these vertices are potential locations for local shape descriptors. We select all 549 vertices as feature locations. Obviously there is a memory-performance trade off at setting the number of the features. In section 4.5, we have fitted the generic mesh model on 400x320 uniform grid having $0.5 \mathrm{~mm}$ resolution in $\mathrm{X}, \mathrm{Y}$ plane interpolated/resampled $\mathrm{Z}$ values. All shape maps are calculated on this 400x320 uniform grid and therefore are also sized 400x320. To sum up, we generate descriptors at fixed scale and fixed orientation on 3D shape maps at 549 nodes of generic face model. SIFT descriptors are 128 dimensional vectors representing orientation histograms of the image patch at the affinity of interest points ( nodes of generic model in our case). SURF descriptor represent similar information with a vector of 64 dimensions. Two sample shape index maps with SIFT descriptors are given in Figure 4.13 and Figure 4.14. Descriptors at the Figures 4.13 and 4.14 have the size of 16x16 pixels ( $8 \mathrm{~mm} \times 8 \mathrm{~mm}$ metric size for the given grid resolution of $0.5 \mathrm{~mm} /$ pixel). 


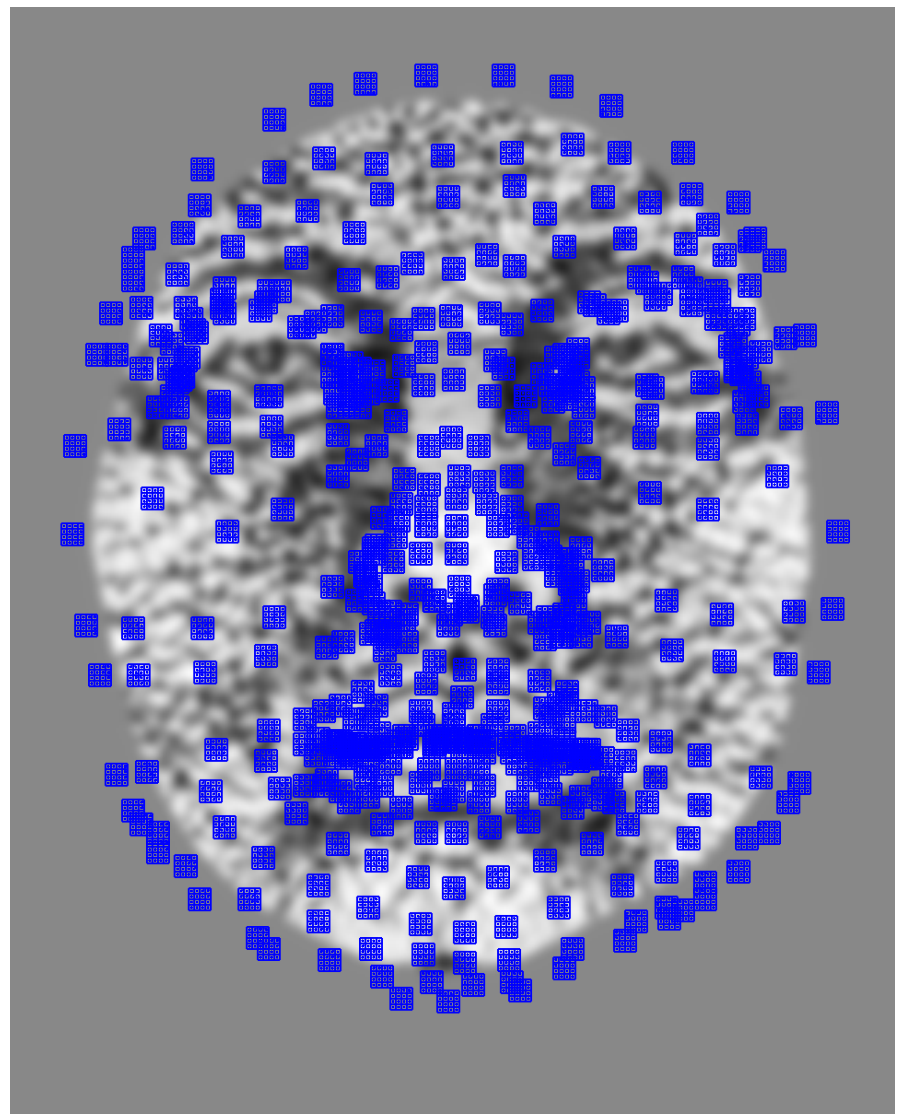

Figure 4.13: SIFT Descriptors calculated on shape index map at 549 nodes of generic model (Sample 1) 


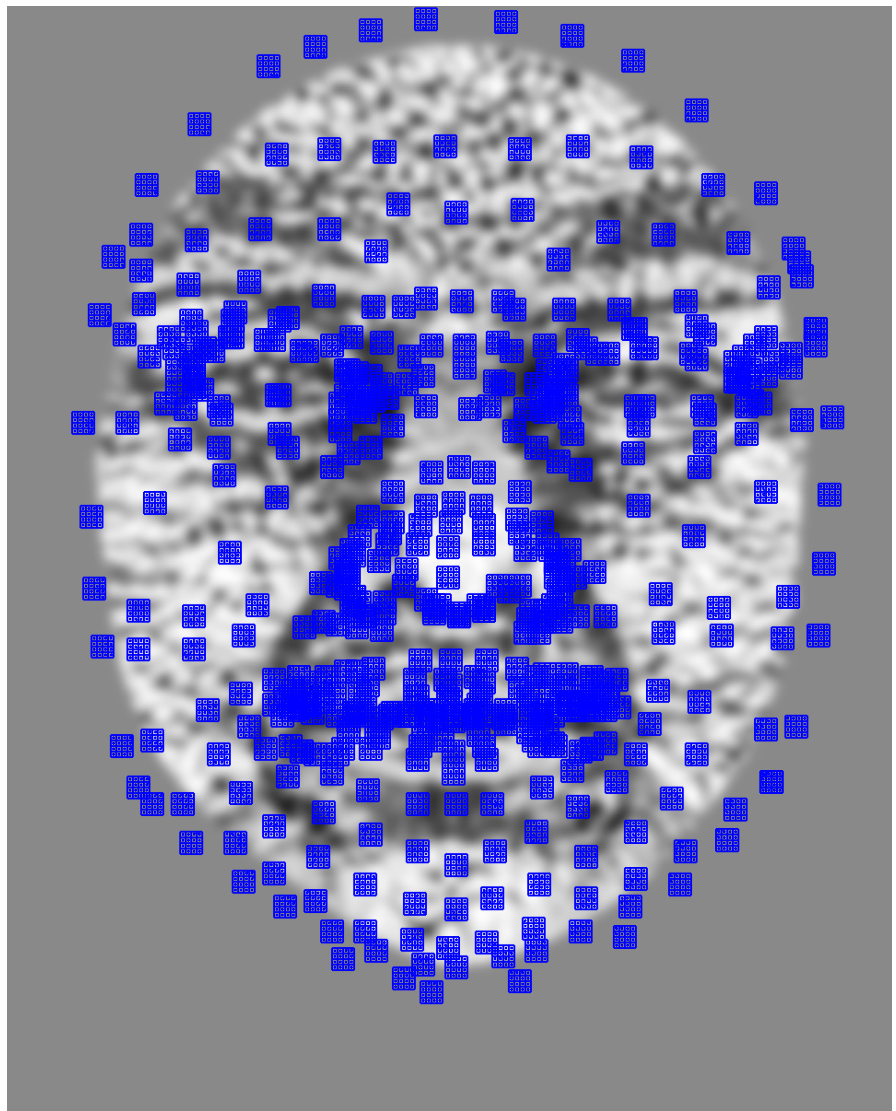

Figure 4.14: SIFT Descriptors calculated on shape index map at 549 nodes of generic model (Sample 2) 


\subsection{Dimension Reduction and Classification}

Dimension reduction method for dimensional feature vector is Fisher's linear discriminant analysis (LDA) and the similarity metric used for recognition/verification is cosine distance. The process is the same with the process that is described in Sections 3.4 and 3.5 . 


\section{CHAPTER 5}

\section{EXPERIMENTAL RESULTS}

\subsection{FRGC Database and Experiment Protocol}

In this section, first brief information about FRGC database and experiment protocol, which is used in our experiments is given. Then the results of the experiments conducted using the FRGC database and protocol will be presented.

3D FRGC database is provided by the University of Notre Dame. Brief information about the 3D FRGC Database and experiment protocol is given below.

\subsubsection{FRGC Database}

The database consists of two sets : FRGC v1.0 and FRGC v2.0. Outlines of these sets can be stated as follows :

- FRGC v1.0. This set consists of $\mathbf{9 4 3}$ scans of $\mathbf{2 7 5}$ subjects. All records are neutral. Data was collected with a Minolta Vivid 900/910 series 3D scanner. 3D data are given in a $640 \mathrm{x} 480$ grid. Each point in the grid has $\mathrm{X}, \mathrm{Y}, \mathrm{Z}$ coordinates in millimeters and a valid flag as well. For each 3D scan, there also exists an accompanying $640 * 4802 \mathrm{D}$ color image. FRGC v1.0 is used as the training set in FRGC Experimental protocol [38].

- FRGC v2.0. This set consist of $\mathbf{4 0 0 7}$ scans of $\mathbf{4 6 6}$ subjects. There are neutral and non-neutral recordings. Data was collected with a Minolta Vivid 900/910 series 3D scanner. 3D data is given in a $640 \times 480$ grid. Each point in the grid 
has $\mathrm{X}, \mathrm{Y}, \mathrm{Z}$ coordinates in millimeters and a valid flag as well. For each 3D scan, there also exists an accompanying $640^{*} 4802 \mathrm{D}$ color image. FRGC v2.0 is used as the validation set in FRGC Experimental protocol .

\subsubsection{FRGC Experiment Protocol}

Experiments in this study are conducted with principals of FRGC experiment protocol and reported accordingly. We can summarize experiment conditions for FRGC protocol as follows :

- Training set. Spring2003 session (943 records of 275 subjects) is used as the training set.

- Testing set. Fall2003 and Spring2004 sessions (4007 records of 466 subjects) are used as the test set.

- All vs All Verification Experiment. All 4007 records are used as targets and probes. A 4007-by-4007 full similarity matrix is obtained and self-matches are neglected. Detection rate at 0.001 false alarm rate is usually reported in the literature as a general performance criterion.

- ROC3 Verification Experiment. Target and query records are from different semesters. Due to the time gap between the sessions, this is the hardest problem in the experiment protocol.

\subsection{Baseline Experiments}

We start our experiments with testing a simplified version of face recognition system given in Figure 3.1. Proposed system is simplified by removing Shape Maps and SIFT/SURF descriptors, and PCA/LDA is directly applied to the range images. Simplified version of the proposed face recognition system is shown in Figure 5.1. 


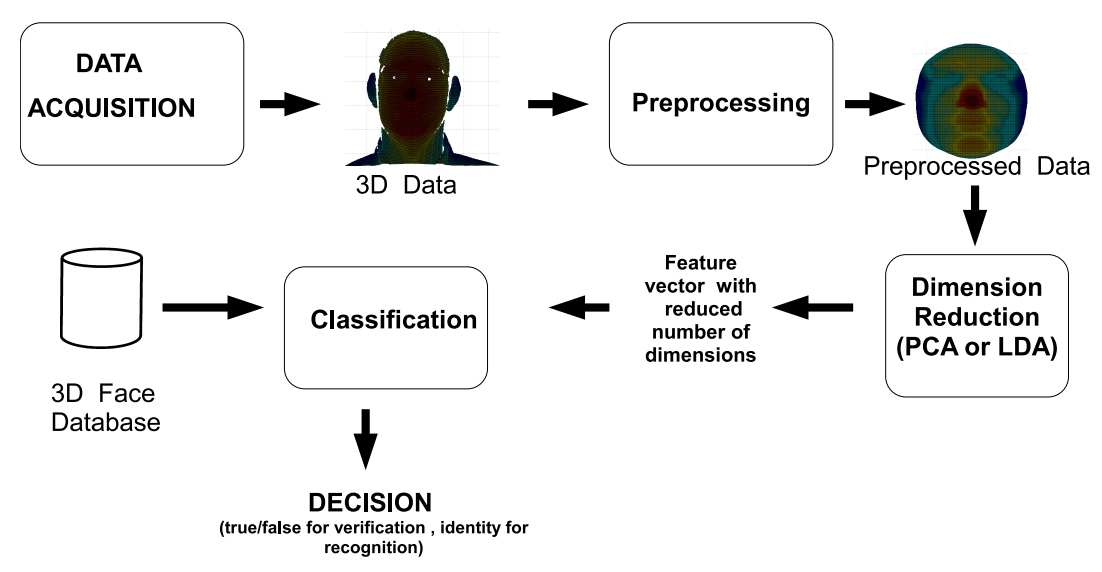

Figure 5.1: Block diagram of the simplified 3D face recognition system for RANGEPCA and RANGE-LDA baseline experiments

\subsubsection{Principal Component Analysis on Range Images (RANGE-PCA)}

First baseline experiment has principal component analysis for the dimension reduction on range images. This experiment uses FRGC v1.0 as the training set and FRGC v2.0 set as the test set. Final dimension of PCA is set to 274 dimensions. Verification result for All vs All and ROC3 experiments is displayed in Figure 5.2. Figure 5.3 displays the verification rates at neutral vs neutral, neutral vs non-neutral, and nonneutral vs non-neutral subsets of similarity matrix. In Figure 5.4, Cumulative Match Characteristics of the experiment is given.

\subsubsection{Linear Discriminant Analysis on Range Images (RANGE-LDA)}

Second baseline experiment has linear discriminant analysis for the dimension reduction on range images. This experiment uses FRGC v1.0 as the training set and FRGC v2.0 set as the test set. Final dimension of LDA is set to 274 dimensions. Verification result for All vs All and ROC3 experiments is displayed in Figure 5.5. Figure 5.6 displays the verification rates at neutral vs neutral, neutral vs non-neutral, and non- 


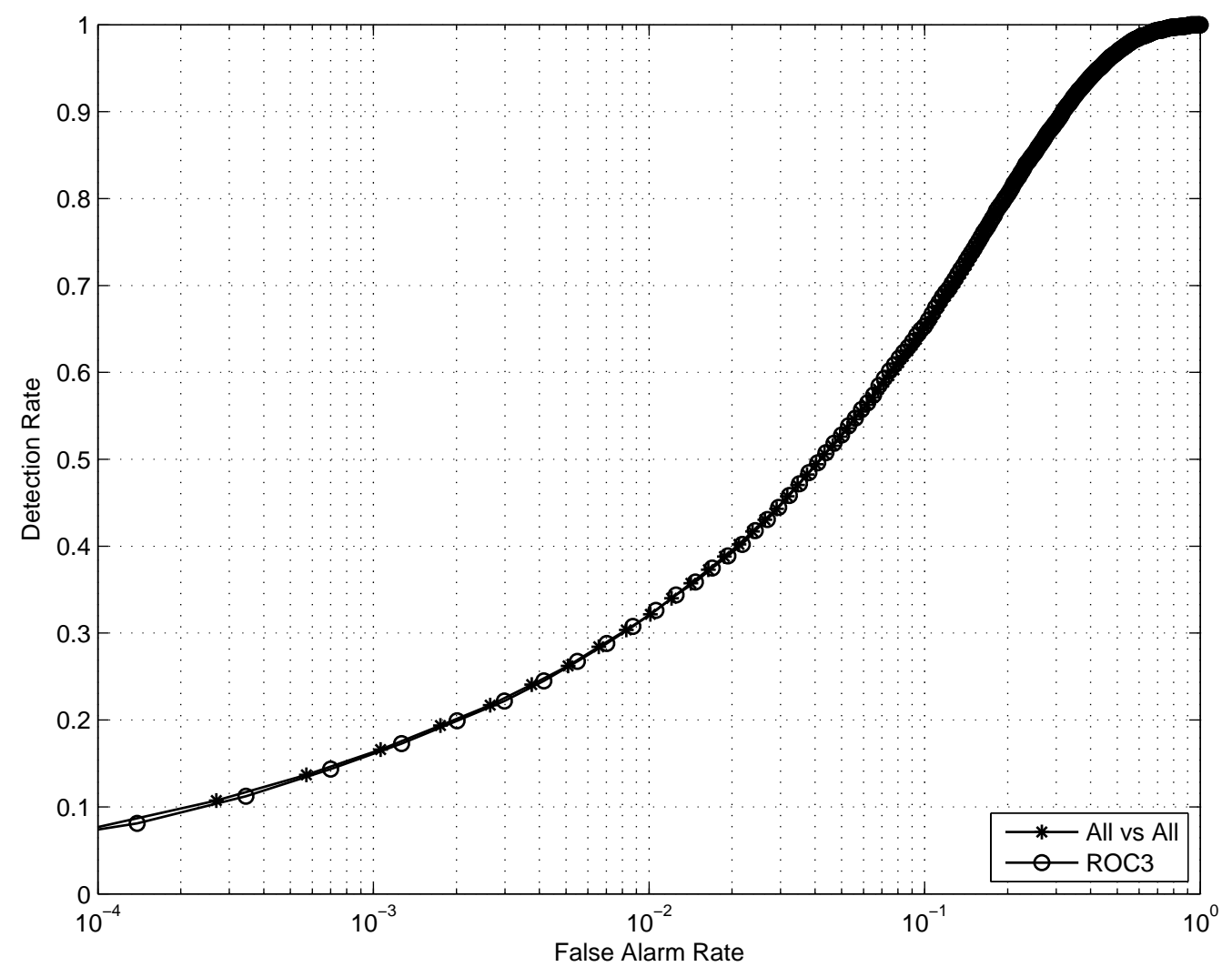

Figure 5.2: ROC curves for PCA applied on range images (All vs All, ROC3) (RANGE-PCA) 


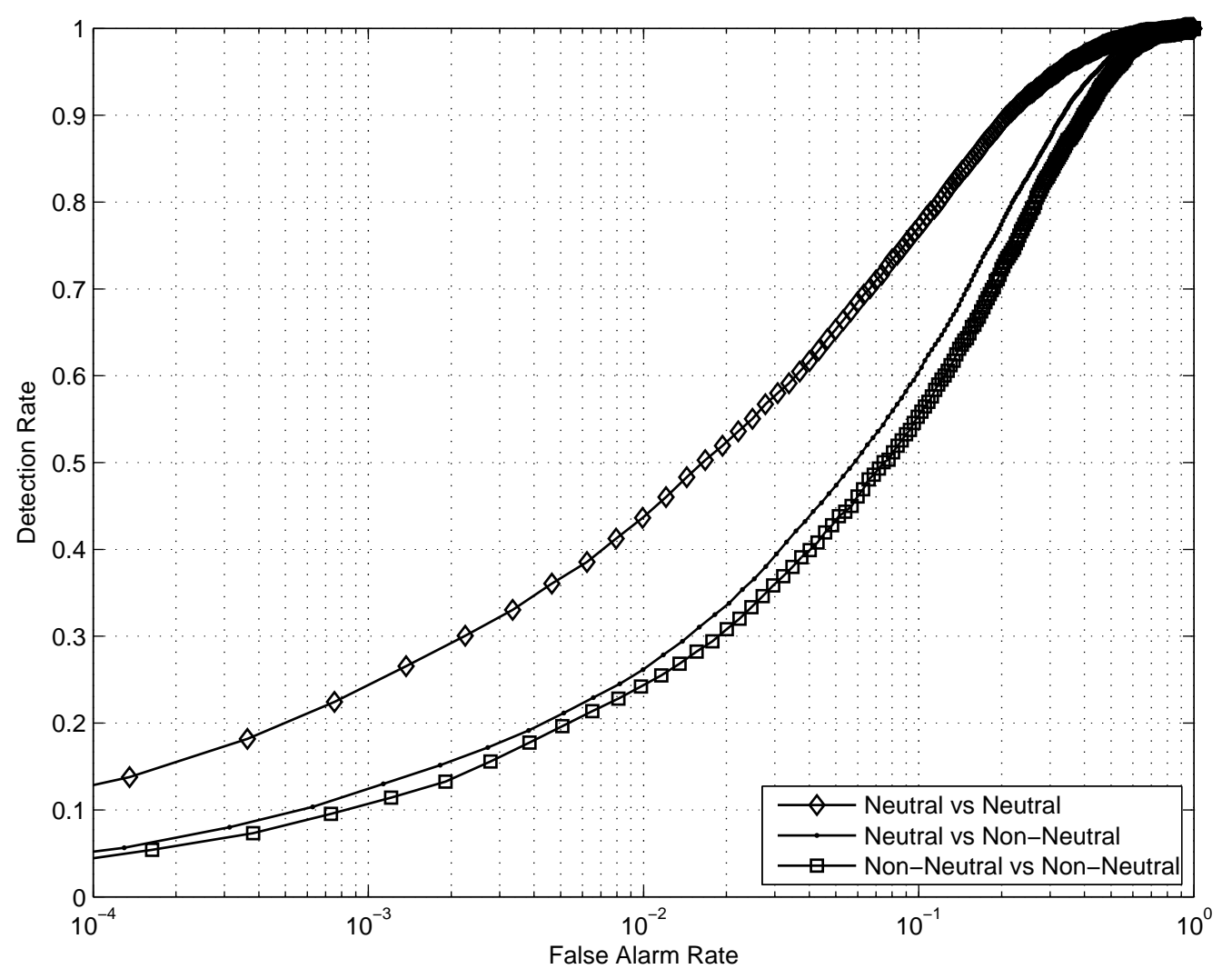

Figure 5.3: ROC curves for PCA applied on range images(Neutral vs Neutral, Neutral vs Non-Neutral, Non-Neutral vs Non-Neutral) (RANGE-PCA) 


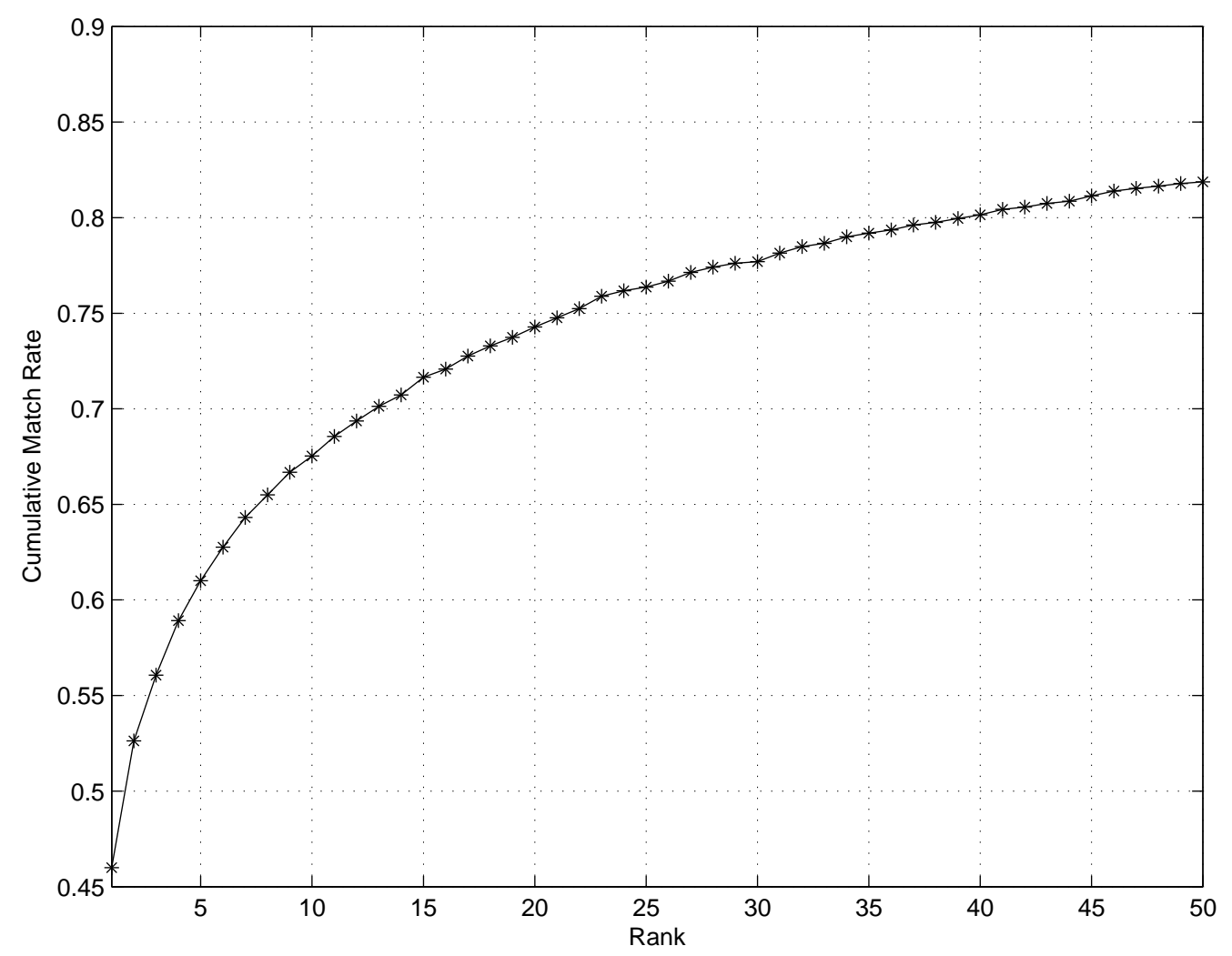

Figure 5.4: Cumulative Match Characteristics for PCA applied on range images(RANGE-PCA) 
neutral vs non-neutral subsets of similarity matrix. In Figure 5.7, Cumulative Match Characteristics of the experiment is given.

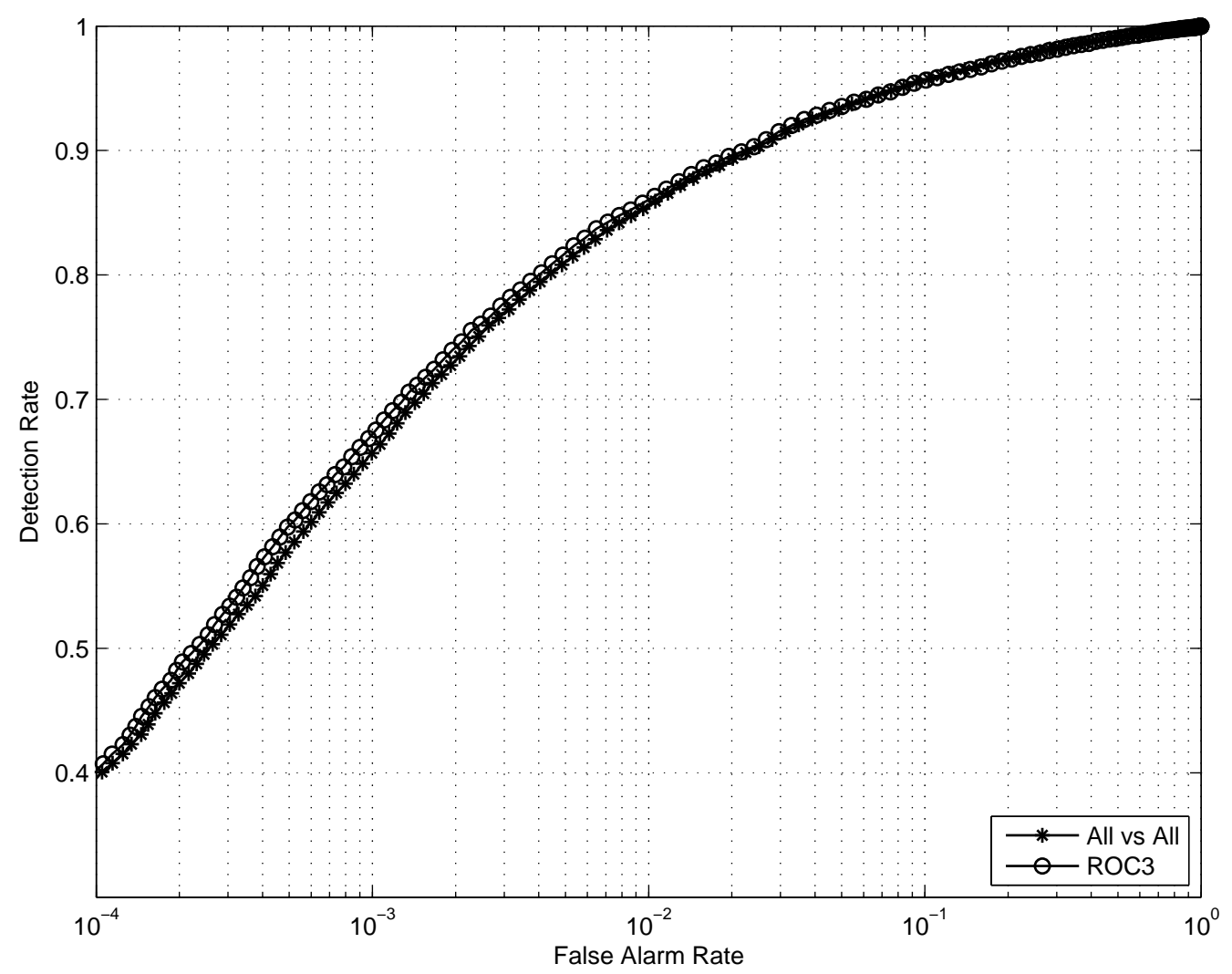

Figure 5.5: ROC curves for LDA applied on range images (All vs All, ROC3) (RANGE-LDA)

\subsubsection{Principal Component Analysis on Shape Maps (MAP-PCA)}

We continue our baseline experiments with testing another simplified version of face recognition system given in Figure 3.1. Proposed system is simplified by removing SIFT/SURF descriptors, and PCA/LDA is directly applied to the shape maps. Simplified version of the proposed face recognition system is shown in Figure 5.8. The simplified experiments will serve as a baseline for the original system and also will give an intuition about the usefulness of the SIFT/SURF descriptors.

First group of baseline experiments in this section have principal component analysis 


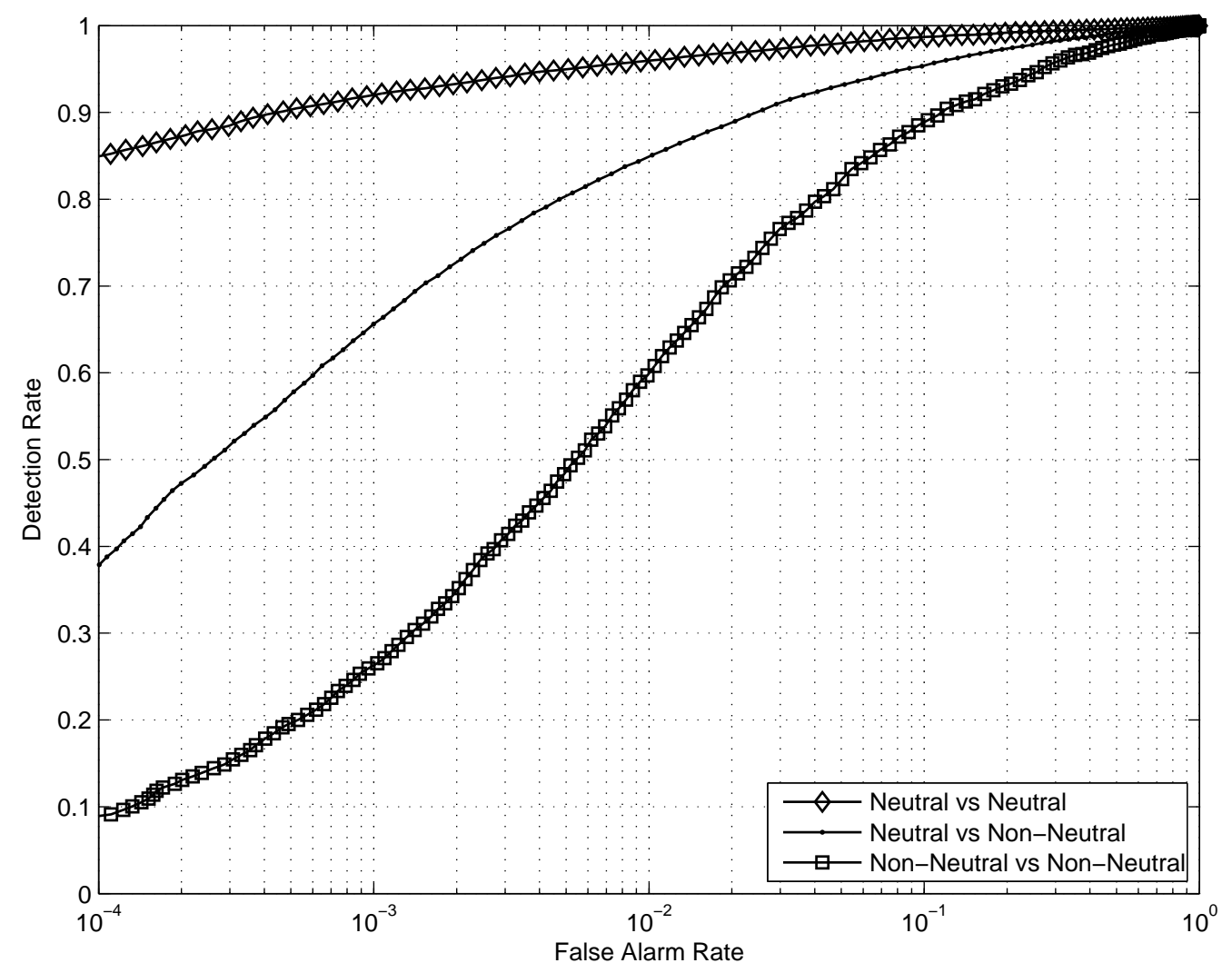

Figure 5.6: ROC curves for LDA applied on range images(Neutral vs Neutral, Neutral vs Non-Neutral, Non-Neutral vs Non-Neutral) (RANGE-LDA) 


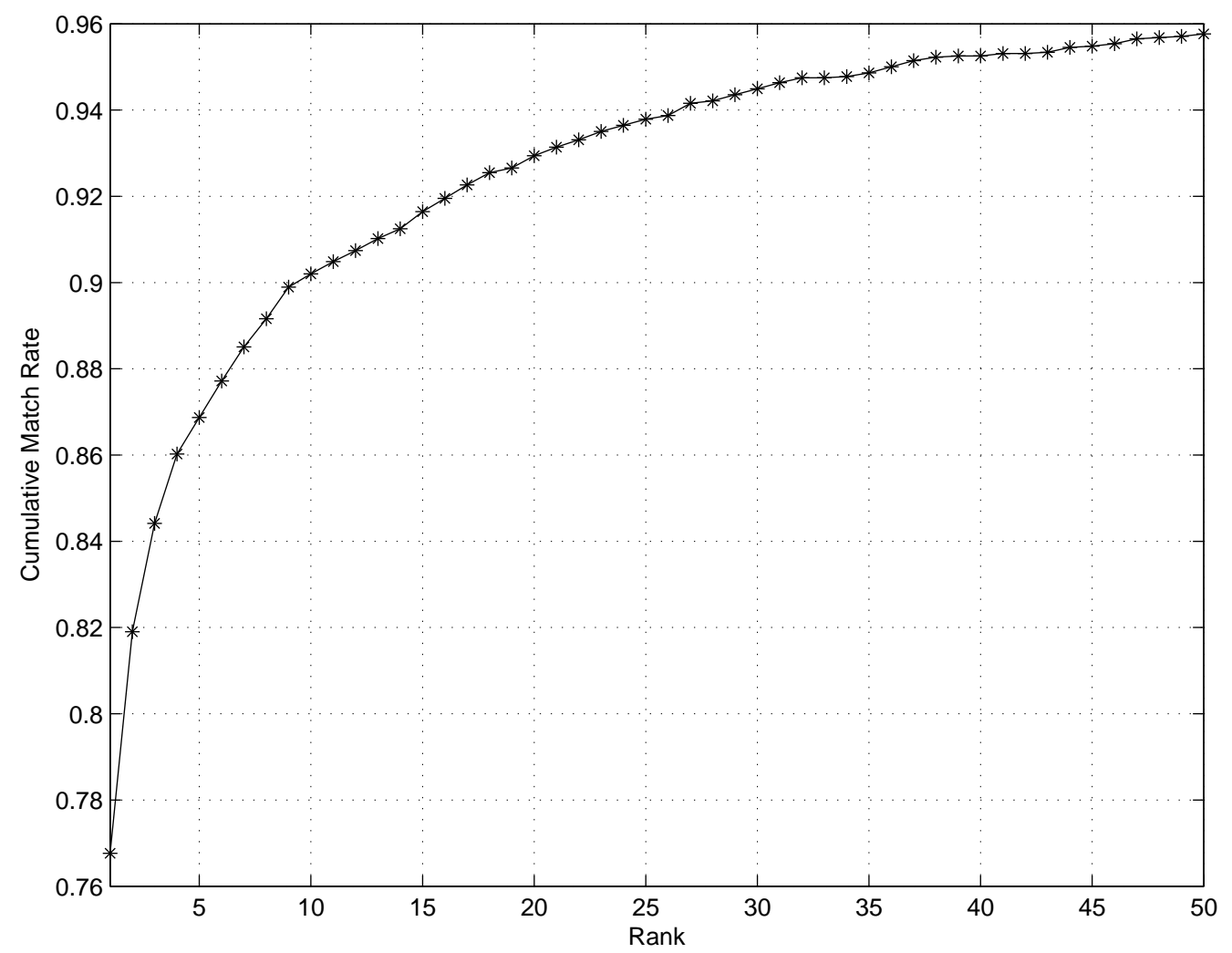

Figure 5.7: Cumulative Match Characteristics for LDA applied on range images(RANGE-LDA) 


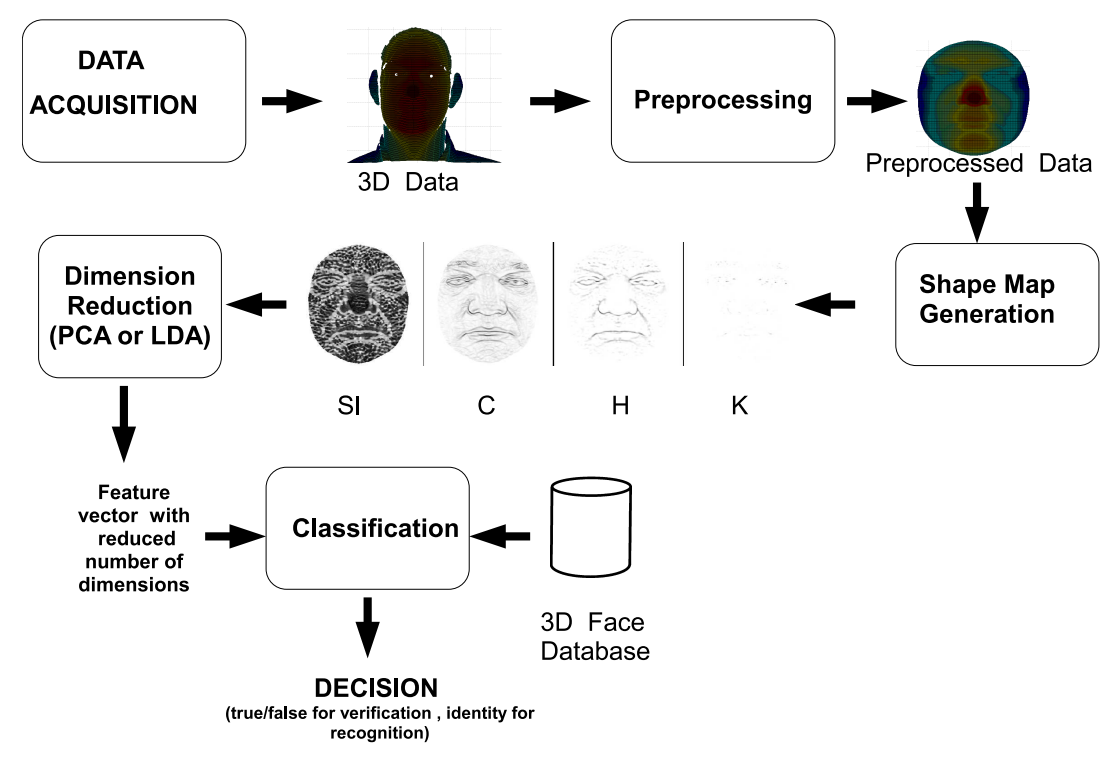

Figure 5.8: Block diagram of the simplified 3D face recognition system for MAP-PCA and MAP-LDA baseline experiments

for the dimension reduction. There are four types of shape maps, hence four experiments are performed in this group. All experiments use FRGC v1.0 as the training set and FRGC v2.0 set as the test set. Final dimension of PCA is set to 274 dimensions. Verification results for All vs All and ROC3 experiments are displayed in Figure 5.9. All vs All detection rates for $0.1 \%$ false alarm rate are summarized in Table 5.1. Figure 5.10 displays the verification rates at neutral vs neutral, neutral vs non-neutral, and non-neutral vs non-neutral subsets of similarity matrix. In Figure 5.11, Cumulative Match Characteristics of the experiments are given.

Table 5.1: Detection Rates for All vs All Experiment at 0.001 False Alarm Rate of Baseline 3D Face Recognition System with Shape Map + PCA (MAP-PCA)

\begin{tabular}{ccc}
\hline $\begin{array}{c}\text { Shape Map } \\
\text { SI }\end{array}$ & Dimension Reduction & DR\% \\
\hline C & PCA & 52.6 \\
\hline H & PCA & 15.9 \\
\hline K & PCA & 16.1 \\
\hline
\end{tabular}



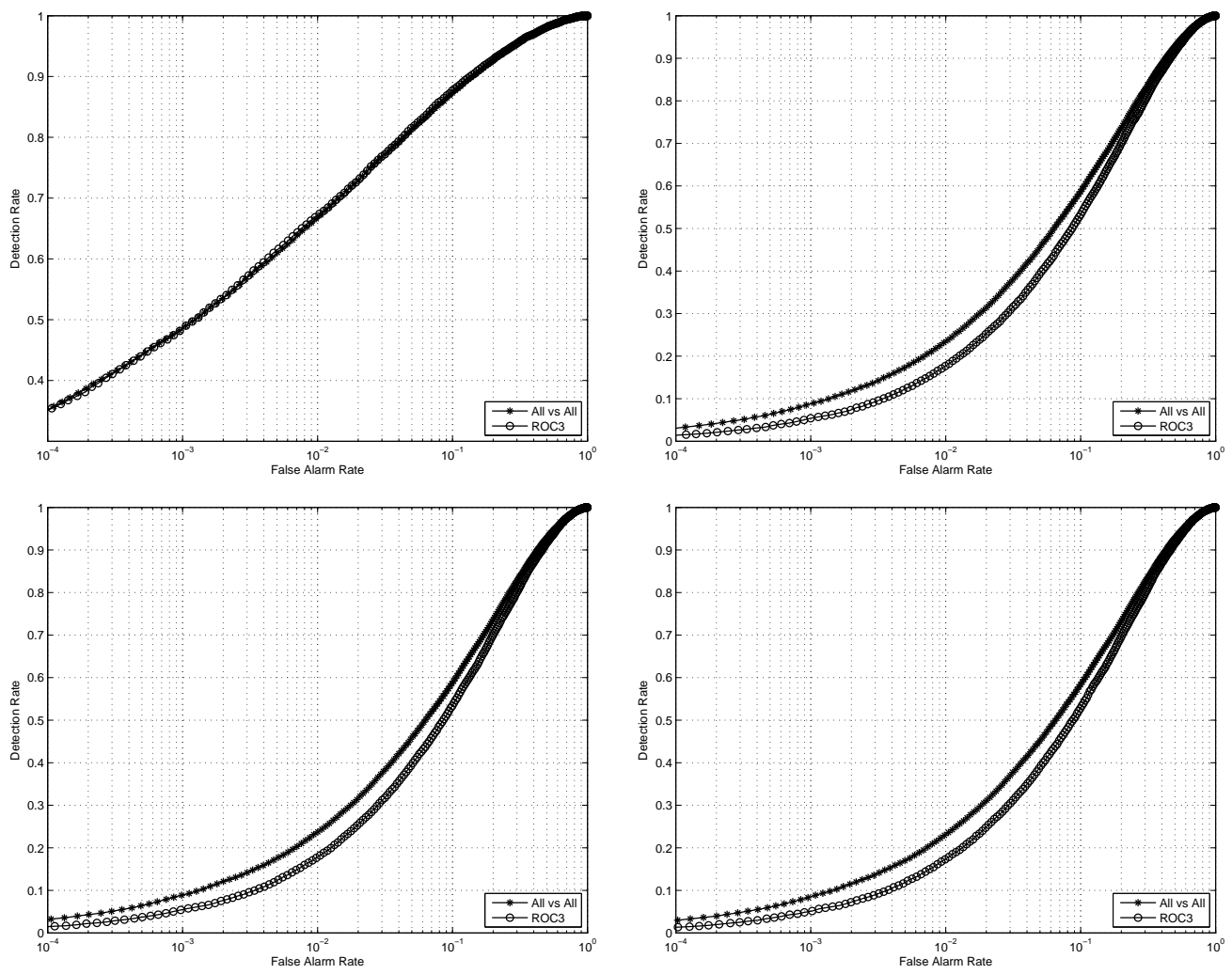

Figure 5.9: ROC curves for SI, C (top row) and H, K (bottom row) Shape Maps with PCA (All vs All, ROC3) (MAP-PCA) 

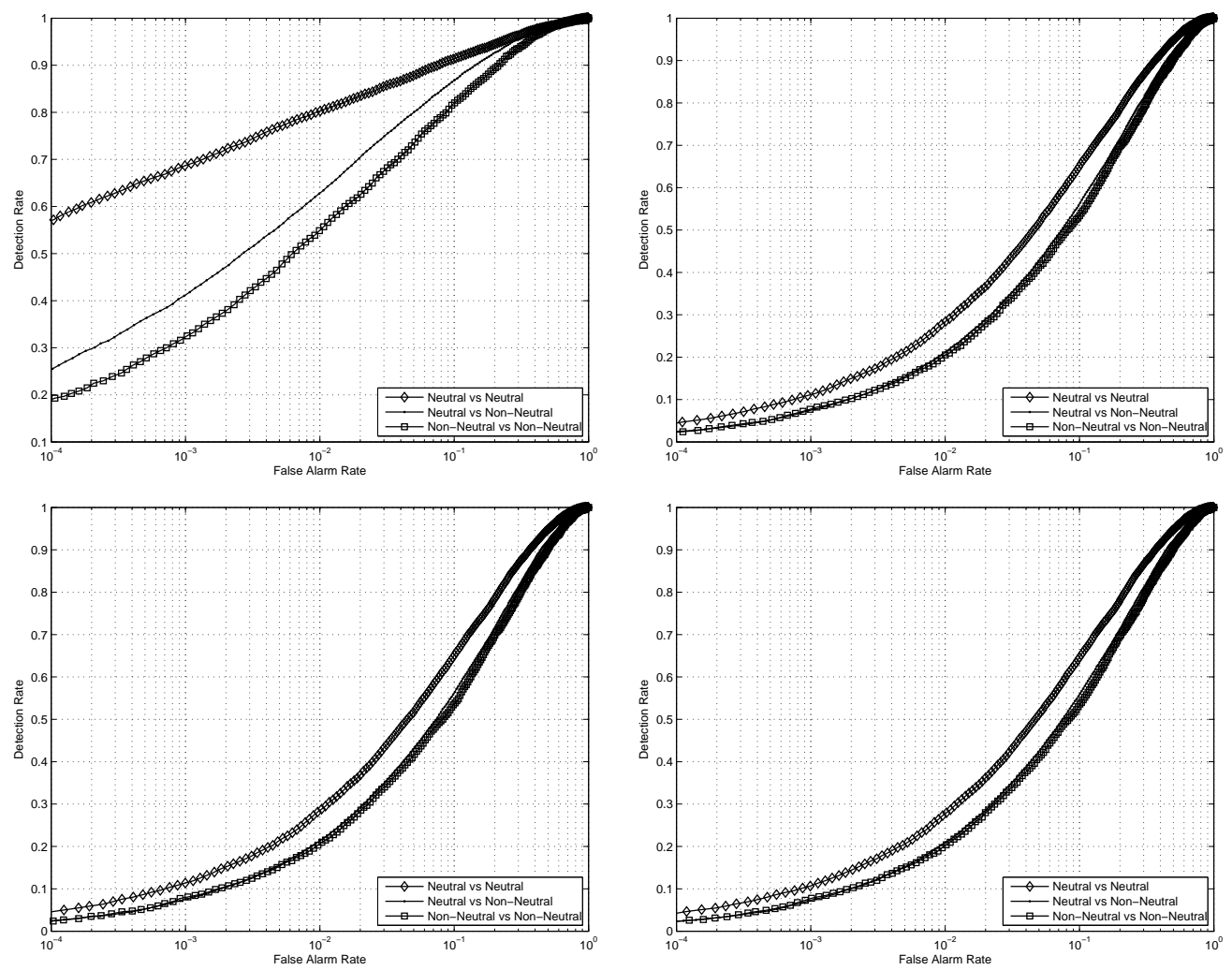

Figure 5.10: ROC curves for SI, C (top row) and H, K (bottom row) Shape Maps with PCA (Neutral vs Neutral, Neutral vs Non-Neutral, Non-Neutral vs Non-Neutral) (MAP-PCA) 

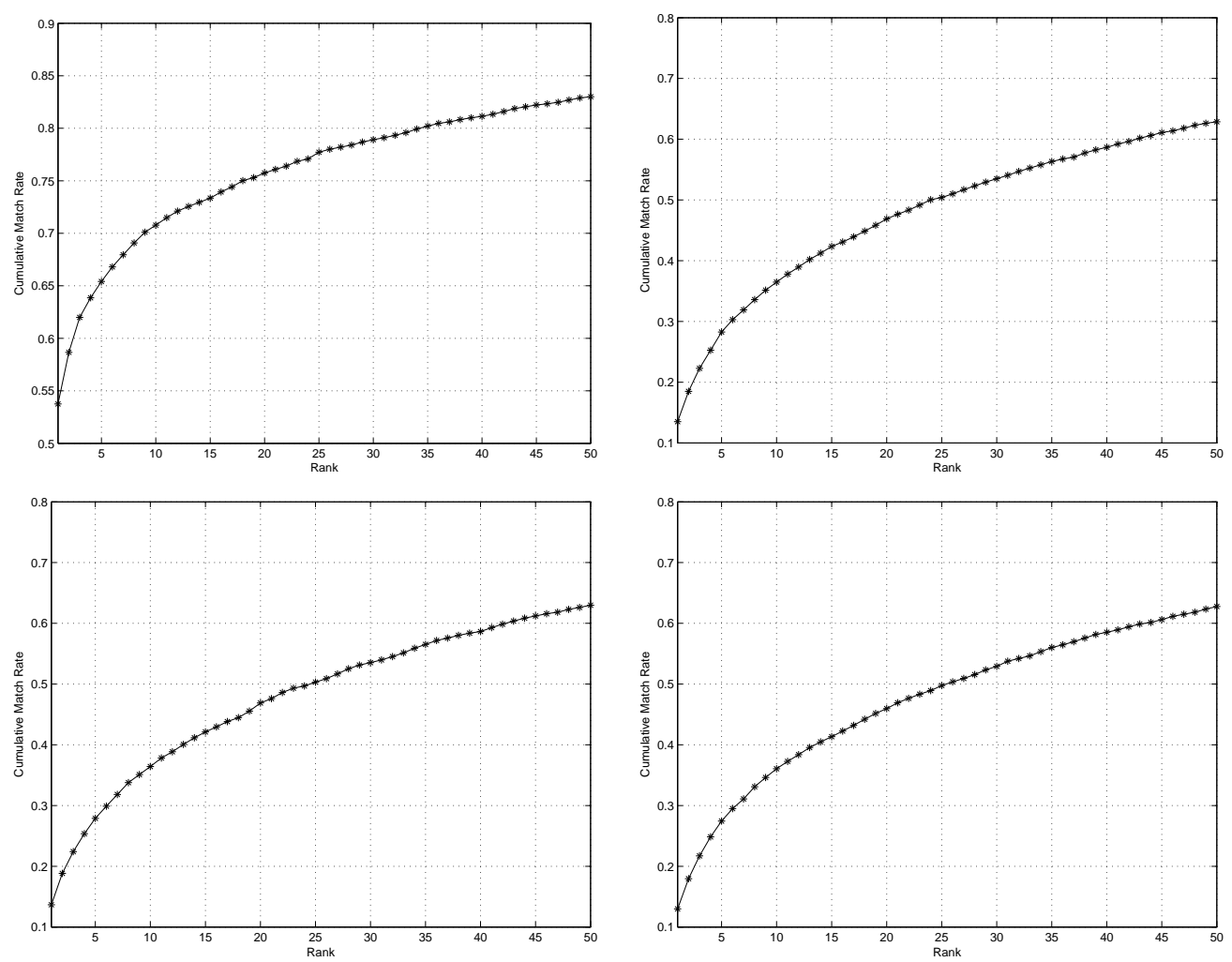

Figure 5.11: Cumulative Match Characteristics for SI, C (top row) and H, K (bottom row) Shape Maps with PCA (MAP-PCA) 


\subsubsection{Linear Discriminant Analysis on Shape Maps (MAP-LDA)}

Second group of baseline experiments in this section have linear discriminant analysis for the dimension reduction. There are four types of shape maps, hence four experiments are performed in this group. All experiments use FRGC v1.0 as the training set and FRGC v2.0 set as the test set. FRGC v1.0 has 275 subjects ( $n=275$ classes), therefore the LDA generates a subspace with $n-1=274$ dimensions. Verification results for All vs All and ROC3 experiments are displayed in Figure 5.12. All vs All detection rates for $0.1 \%$ false alarm rate are summarized in Table 5.2. Figure 5.13 displays the verification rates at neutral vs neutral, neutral vs non-neutral, and non-neutral vs non-neutral subsets of similarity matrix. In Figure 5.14, Cumulative Match Characteristics of the experiments are given.

Results of baseline experiments show that shape index + LDA combination gives the best result with $73.8 \%$ detection rate at $0.1 \%$ false alarm rate for All vs All experiment. However even this perfomance figure is cconsiderably lower than the performance values displayed in recently published papers.

Table 5.2: Detection Rates for All vs All Experiment at 0.001 False Alarm Rate of Baseline 3D Face Recognition System with Shape Map + LDA (MAP-LDA)

\begin{tabular}{ccc}
\hline $\begin{array}{c}\text { Shape Map } \\
\text { SI }\end{array}$ & $\begin{array}{c}\text { Dimension Reduction } \\
\text { LDA }\end{array}$ & $\begin{array}{c}\text { DR\% } \\
\text { C }\end{array}$ \\
\hline H & LDA & 73.8 \\
\hline K & LDA & 24.0 \\
\hline
\end{tabular}

\subsection{Experimental Results for Automatic 3D Face Recognition Using Uniformly Distributed Shape Descriptors}

\subsubsection{Evaluation of different shape maps and descriptor sizes (UNI-EVA)}

We have performed experiments on FRGC database to test the performance of the 3D face recognition system. According to FRGC testing protocol, training set consists 

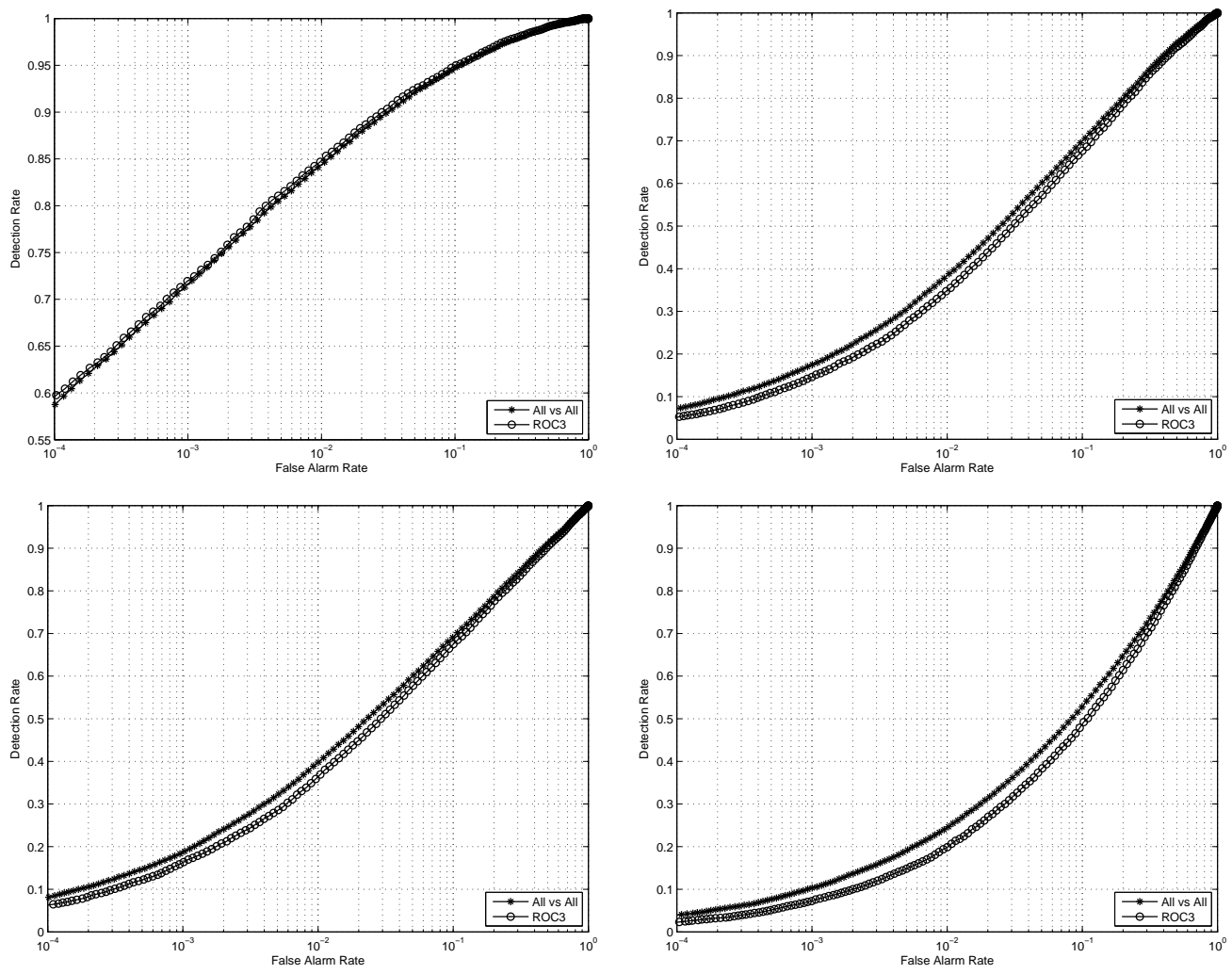

Figure 5.12: ROC curves for SI, C (top row) and H, K (bottom row) Shape Maps with LDA (All vs All, ROC3) (MAP-LDA) 

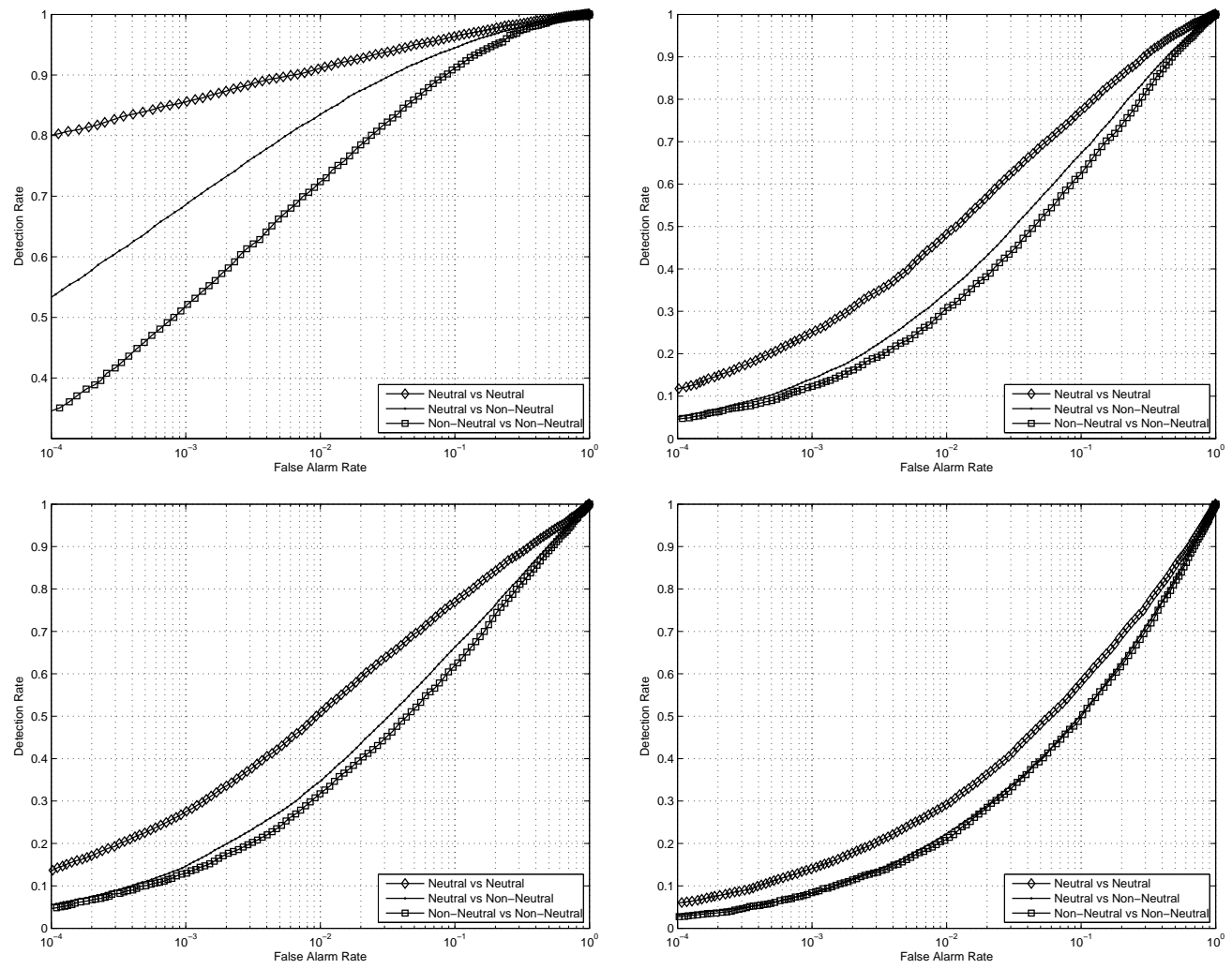

Figure 5.13: ROC curves for SI, C (top row) and H, K (bottom row) Shape Maps with LDA (Neutral vs Neutral, Neutral vs Non-Neutral, Non-Neutral vs Non-Neutral) (MAP-LDA) 

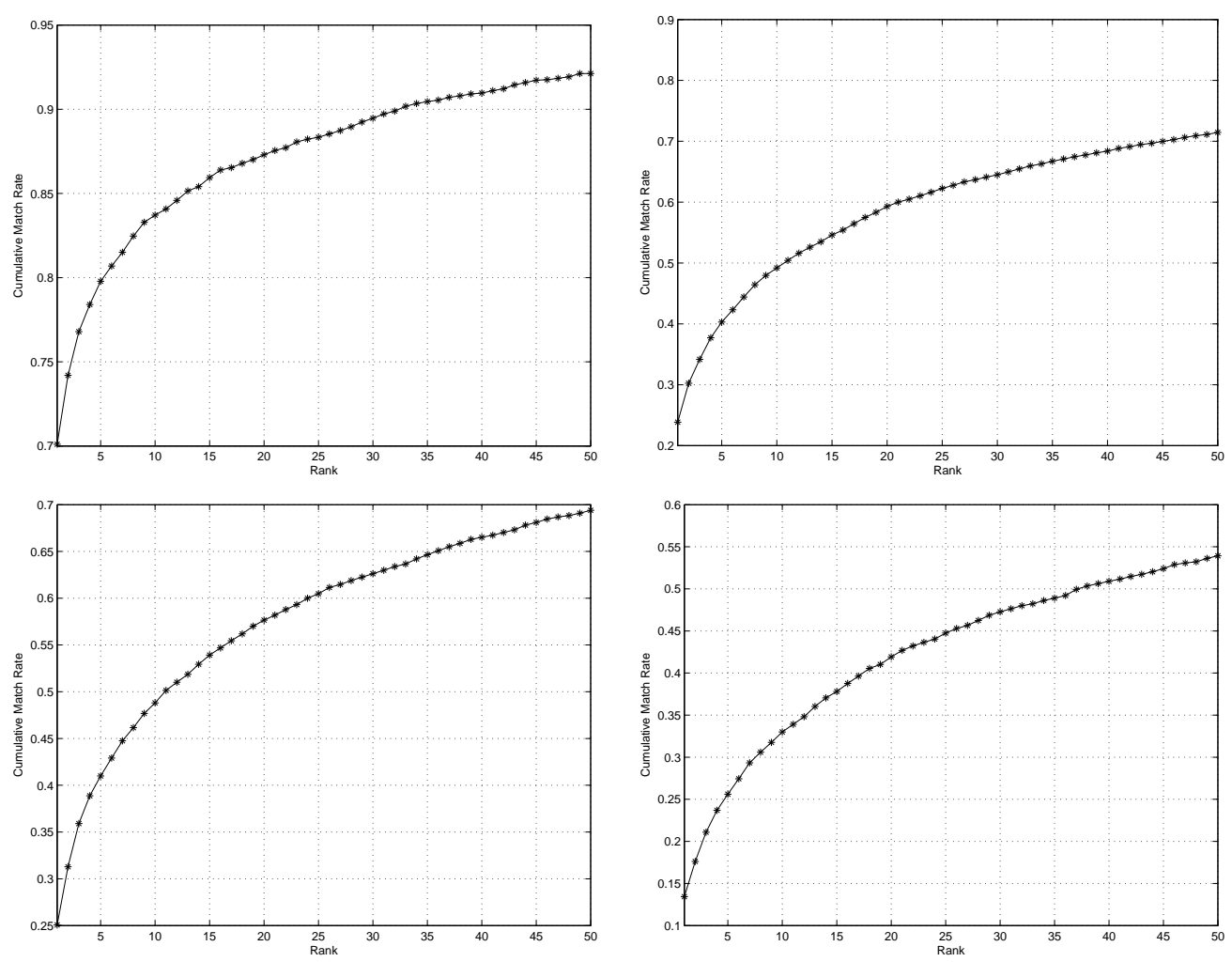

Figure 5.14: Cumulative Match Characteristics for SI, C (top row) and H, K (bottom row) Shape Maps with LDA (MAP-LDA) 
of 943 records of 275 subjects (FRGCv1.0). Testing set has 4007 records of 466 subjects (FRGCv2.0). We performed recognition experiments with SIFT descriptors of different sizes. Descriptor sizes used throughout the experiments were 4,8,12,16, 32 millimeters. For the SURF descriptors, gaussian standard deviation, $\sigma$, had the values of $1,2,3,4,5$ millimeters representing different scales. Smaller sized descriptors focus more on local variations, whereas larger scales correspond to global shape variations.

We have performed experiments on FRGCv2.0 3D database for different shape maps and different scales. There are three sets of experiments:

- First set of experiments are performed by using all 457 feature points.

- Second set of experiments are performed by using 229 feature points. For this set, we traced the feature point locations row-wise and picked up one of two feature points.

- Third set of experiments are performed by using 153 feature points. For this set, we traced the feature point locations row-wise and picked up one of three feature points.

For all three sets of experiments, we sticked to the FRGC v1.0 dataset. FRGC v1.0 has 275 subjects and 250 of 275 subjects are used for training and 25 subjects are used for testing. Hence, we perform the experiments eleven times in 11-fold manner. Accordingly, we obtain the mean and standard deviation of the detection rates. Also we should note that using 250 subjects (i.e. $n=250$ classes) makes the LDA generate a subspace with $n-1=249$ dimensions. Mean detection rates and standard deviations with 457, 229 and 153 feature points for All vs All experiment at 0.001 false alarm rate are given in Table 5.3. 


\subsubsection{Combined Feature Experiment with 5 Selected Shape Map - De- scriptor Combinations (UNI-CF5D)}

Best five scores for the second set of experiments are displayed in bold fonts in Table 5.3. The features in these rows are selected for designing a combined-feature 3D recognition system. In fact, there are better performance figures in the first experiment. However, in the first experiment all 457 features are used hence memory requirement for the first experiment is very high. Therefore we consider the number of features in the second experiment is the best candidate for designing a multi-scale recognition system in terms of memory-performance trade off. We generate a combined feature vector by concatenating selected feature vectors. Combined feature vector has a dimension of $5 \times 229 \times 128$. We perform an experiment using combined feature vectors. This experiment has FRGC v1.0 dataset(275 subjects) as the training dataset, and FRGC v2.0 dataset(466 subjects) as testing data. FRGC v1.0 has 275 subjects ( $n=275$ classes), therefore the LDA generates a subspace with $n-1=274$ dimensions. Receiver operating characteristics curves for the experiment are given in Figure 5.15, Figure 5.16. Figure 5.15 displays the verification rates in All vs All and ROC3 experiments. Figure 5.16 displays the the verification rates at neutral vs neutral, neutral vs non-neutral, and non-neutral vs non-neutral subsets of similarity matrix. In Figure 5.17, Cumulative Match Characteristics of the experiment is given. In Figure 5.15, we observe that we have obtained $97.33 \%$ detection rate at $0.1 \%$ false alarm rate (FAR) for All vs All experiment.

\subsubsection{Combined Feature Experiment with Multi Shape Map - Multi Scale SIFT Descriptors (UNI-CFMD)}

We generate a multi shape map - multi scale feature vector by concatenating all possible SIFT feature vectors. Combined feature vector has a dimension of 5 (scales) $\mathrm{x}$ 4 (shape maps) x 229(locations) x 128(SIFT feature size). We perform an experiment using combined feature vectors. This experiment has FRGC v1.0 dataset(275 subjects) as the training data, and FRGC v2.0 dataset(466 subjects) as the testing data. FRGC v1.0 has 275 subjects ( $n=275$ classes), therefore the LDA generates a subspace with $n-1=274$ dimensions. Figure 5.18 displays the verification rates in All vs All 
Table 5.3: Mean Detection Rates with Standard Deviations for All vs All Experiment at 0.001 False Alarm Rate of Automatic Model Supported 3D Face Recognition System for 457, 229 and 153 feature points (UNI-EVA)

\begin{tabular}{|c|c|c|c|c|c|}
\hline Descriptor & Shape Map & $\begin{array}{l}\text { Size } \\
(\mathrm{mm})\end{array}$ & $\begin{array}{c}\mathbf{D R} \pm \mathbf{s t d} \\
(457 \text { points })\end{array}$ & $\begin{array}{c}\mathbf{D R} \pm \text { std } \\
(229 \text { points })\end{array}$ & $\begin{array}{c}\mathbf{D R} \pm \mathbf{s t d} \\
(153 \text { points })\end{array}$ \\
\hline \multirow{20}{*}{ SIFT } & \multirow{5}{*}{ SI } & 4 & $89,71 \pm 5,72$ & $89,32 \pm 5,83$ & $89,55 \pm 6,43$ \\
\hline & & 8 & $98,32 \pm 2,07$ & $98,15 \pm 2,57$ & $98.57 \pm 1,99$ \\
\hline & & 12 & $99.38 \pm 1,05$ & $\mathbf{9 9 , 4 8} \pm 0,83$ & $99.39 \pm 0.77$ \\
\hline & & 16 & $98.94 \pm 1.94$ & $98,65 \pm 3,01$ & $98.55 \pm 3.00$ \\
\hline & & 32 & $95,07 \pm 4,97$ & $95,36 \pm 4,71$ & $95,18 \pm 6,05$ \\
\hline & \multirow{5}{*}{$\mathrm{C}$} & 4 & $92,21 \pm 5,95$ & $92,53 \pm 5,96$ & $92,21 \pm 5,81$ \\
\hline & & 8 & $95,24 \pm 4,88$ & $95,07 \pm 5,15$ & $95,05 \pm 5,29$ \\
\hline & & 12 & $91,15 \pm 6,69$ & $92,15 \pm 5,90$ & $90,78 \pm 6.56$ \\
\hline & & 16 & $87,19 \pm 7.38$ & $86,94 \pm 7,39$ & $87.42 \pm 7.47$ \\
\hline & & 32 & $50.57 \pm 11.81$ & $49,03 \pm 12,61$ & $47,84 \pm 12,38$ \\
\hline & \multirow{5}{*}{$\mathrm{H}$} & 4 & $89,94 \pm 6,27$ & $89,89 \pm 6,91$ & $89,43 \pm 6,85$ \\
\hline & & 8 & $97,44 \pm 3,77$ & $\mathbf{9 7 , 4 4 \pm 3 , 7 4}$ & $96,94 \pm 4.77$ \\
\hline & & 12 & $97,89 \pm 2,86$ & $\mathbf{9 7 , 8 4} \pm 3,06$ & $97,65 \pm 2,88$ \\
\hline & & 16 & $96,81 \pm 4,98$ & $96,71 \pm 4,91$ & $96,71 \pm 5,17$ \\
\hline & & 32 & $55,19 \pm 12,62$ & $54,48 \pm 12,25$ & $53,85 \pm 12,2$ \\
\hline & \multirow{5}{*}{ K } & 4 & $89,13 \pm 5,89$ & $89,30 \pm 5,12$ & $89,11 \pm 5,00$ \\
\hline & & 8 & $95,77 \pm 4.45$ & $96,41 \pm 3,28$ & $95,47 \pm 4,48$ \\
\hline & & 12 & $93.70 \pm 5,42$ & $93,10 \pm 5.80$ & $92.77 \pm 6.05$ \\
\hline & & 16 & $89,34 \pm 8,05$ & $88,63 \pm 7,88$ & $88,43 \pm 7,28$ \\
\hline & & 32 & $21,12 \pm 6,68$ & $21,91 \pm 7,06$ & $23,20 \pm 6,86$ \\
\hline \multirow{20}{*}{ SURF } & \multirow{5}{*}{ SI } & 1 & $81,04 \pm 6,00$ & $79,81 \pm 6,64$ & $80,26 \pm 5,89$ \\
\hline & & 2 & $94,81 \pm 4,43$ & $94,67 \pm 4,19$ & $94.31 \pm 4,76$ \\
\hline & & 3 & $97.88 \pm 1.63$ & $97,89 \pm 2,20$ & $97.93 \pm 1.32$ \\
\hline & & 4 & $98.01 \pm 2.48$ & $97,83 \pm 2,37$ & $97.98 \pm 2.26$ \\
\hline & & 5 & $97,28 \pm 2,38$ & $96,50 \pm 3,71$ & $97,01 \pm 2.50$ \\
\hline & \multirow{5}{*}{$\mathrm{C}$} & 1 & $85,88 \pm 7,85$ & $85,51 \pm 6,98$ & $84,61 \pm 6,64$ \\
\hline & & 2 & $92,64 \pm 7,38$ & $92,35 \pm 7,57$ & $92,47 \pm 7,52$ \\
\hline & & 3 & $92,29 \pm 6,97$ & $92,02 \pm 6,77$ & $91,55 \pm 7,50$ \\
\hline & & 4 & $88,84 \pm 8,20$ & $88,89 \pm 8,00$ & $88,60 \pm 9,20$ \\
\hline & & 5 & $84,32 \pm 9,64$ & $84,19 \pm 10,72$ & $83,21 \pm 11,09$ \\
\hline & \multirow{5}{*}{$\mathrm{H}$} & 1 & $80,68 \pm 5,87$ & $80,36 \pm 5,72$ & $80,68 \pm 5,58$ \\
\hline & & 2 & $93,12 \pm 5,79$ & $93,14 \pm 5,84$ & $92,41 \pm 6,92$ \\
\hline & & 3 & $93,13 \pm 5,61$ & $93,67 \pm 5,40$ & $93,47 \pm 5,65$ \\
\hline & & 4 & $90,65 \pm 7,69$ & $90,07 \pm 7,45$ & $91,11 \pm 6,82$ \\
\hline & & 5 & $88,91 \pm 8,39$ & $88,29 \pm 8,66$ & $88,37 \pm 7,99$ \\
\hline & \multirow{5}{*}{$\mathrm{K}$} & 1 & $82,39 \pm 6,27$ & $81,16 \pm 7,74$ & $81,45 \pm 7,28$ \\
\hline & & 2 & $89,42 \pm 7,61$ & $87,83 \pm 9,20$ & $88.74 \pm 7.08$ \\
\hline & & 3 & $82.46 \pm 11.13$ & $82,85 \pm 9,27$ & $82,49 \pm 10,13$ \\
\hline & & 4 & $72,68 \pm 10.88$ & $72,61 \pm 9,91$ & $72,49 \pm 10,72$ \\
\hline & & 5 & $68,76 \pm 12,21$ & $67,96 \pm 11,06$ & $66,10 \pm 11,06$ \\
\hline
\end{tabular}




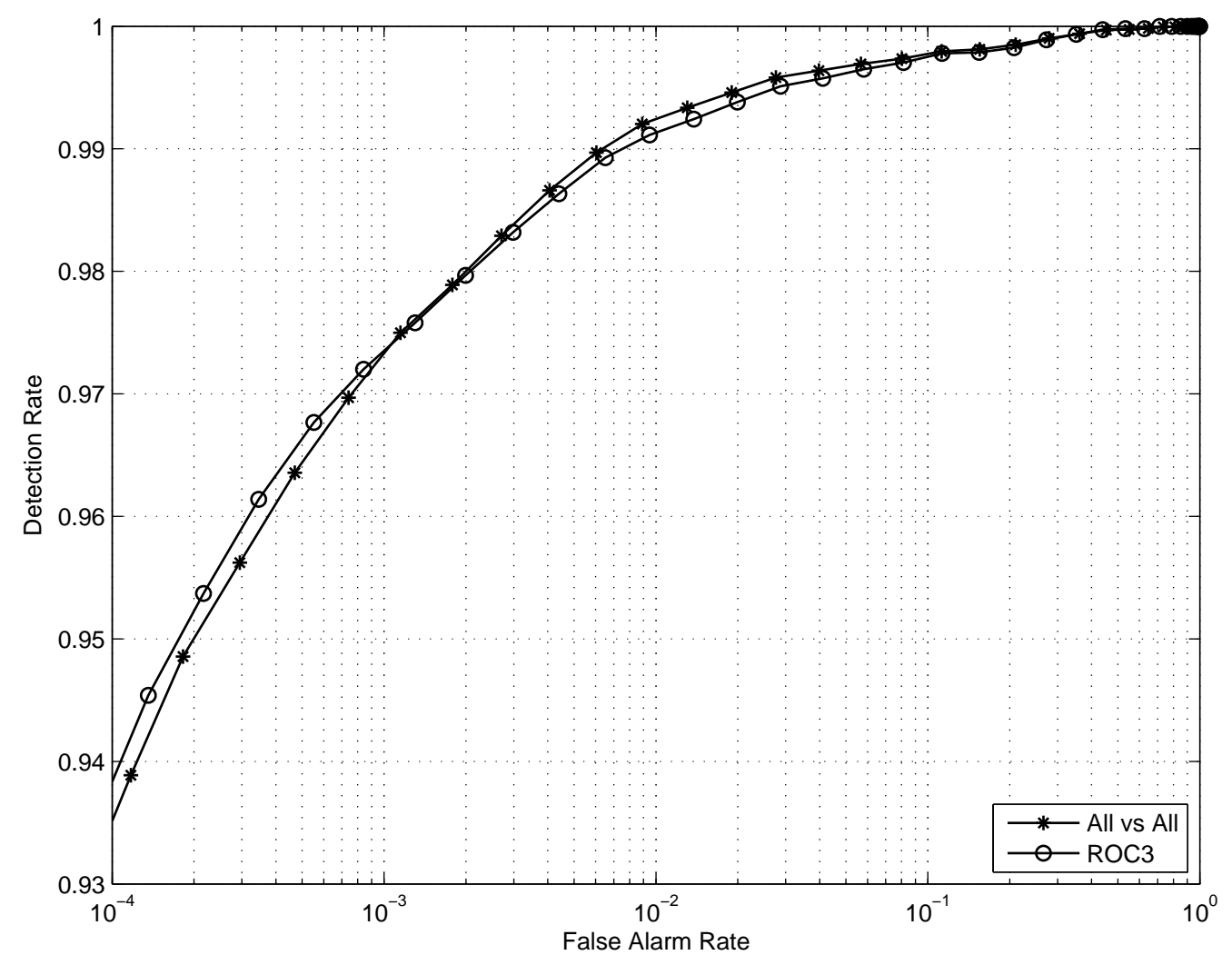

Figure 5.15: ROC curves for 5x229x128 features (All vs All, ROC3) (UNI-CF5D) 


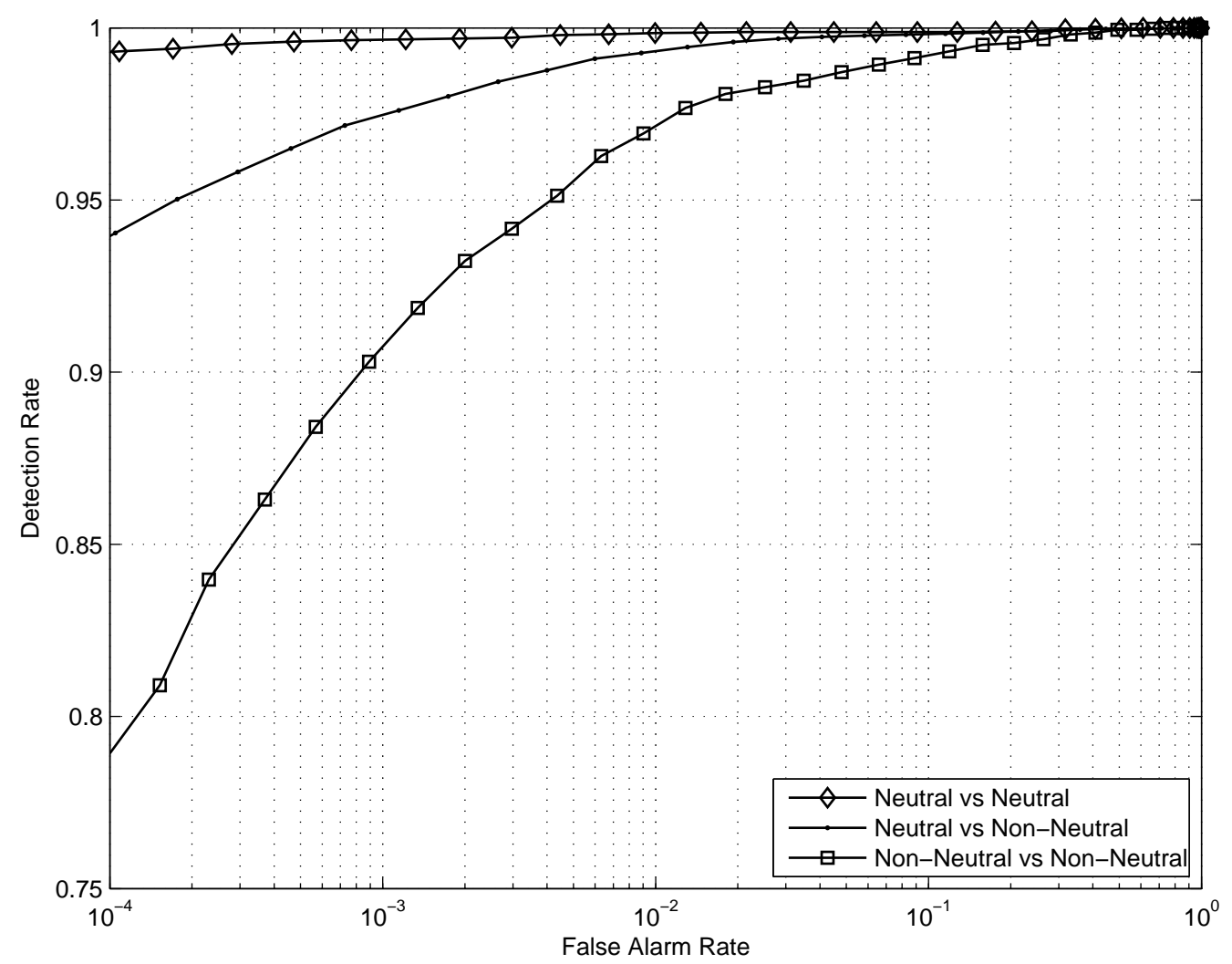

Figure 5.16: ROC curves for 5x229x128 features(Neutral vs Neutral, Neutral vs NonNeutral, Non-Neutral vs Non-Neutral) (UNI-CF5D) 


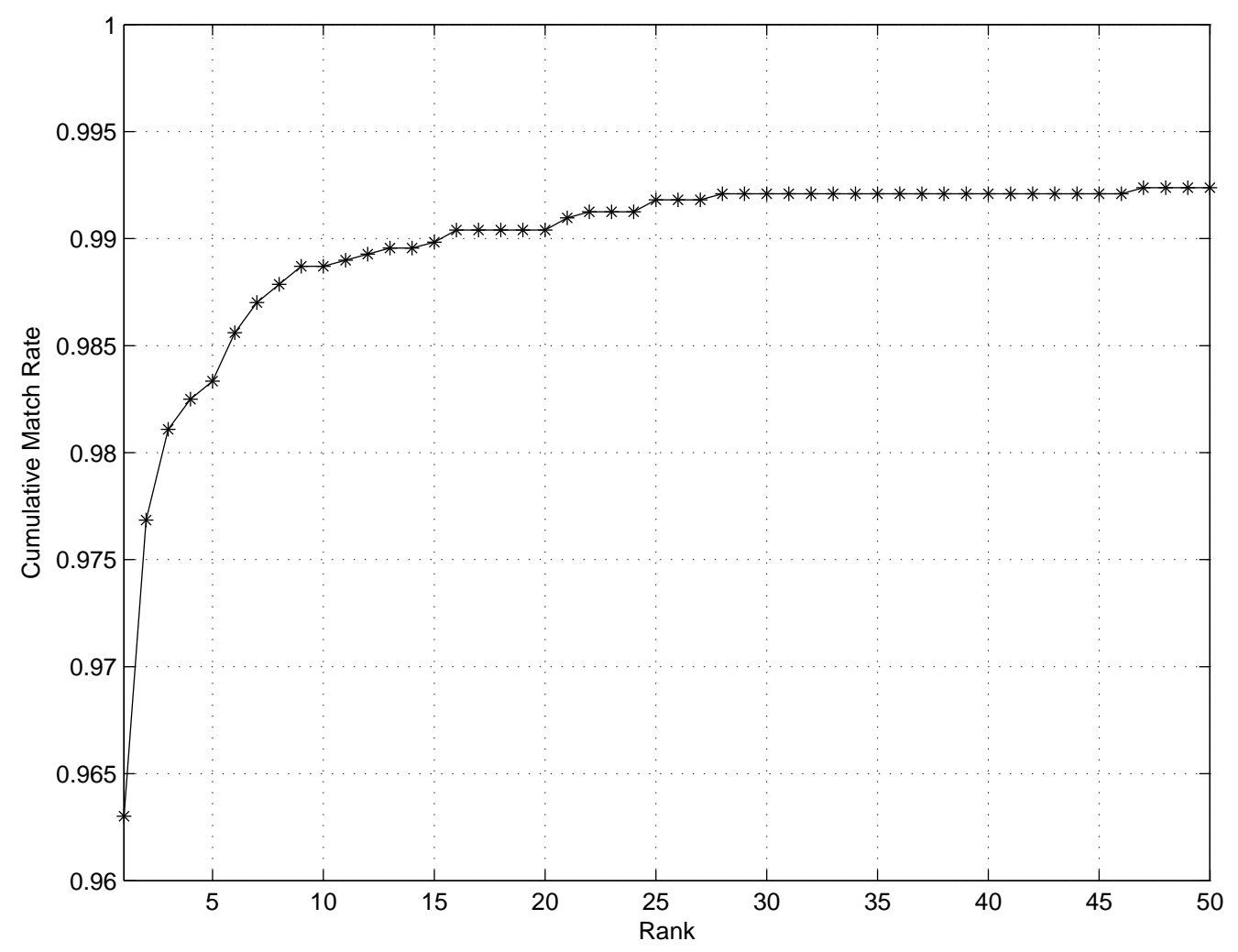

Figure 5.17: Cumulative Match Characteristics for 5x229x128 features (UNI-CF5D) 
and ROC3 experiments. Figure 5.19 displays the the verification rates at neutral vs neutral, neutral vs non-neutral, and non-neutral vs non-neutral subsets of similarity matrix. In Figure 5.20, Cumulative Match Characteristics of the experiment is given.

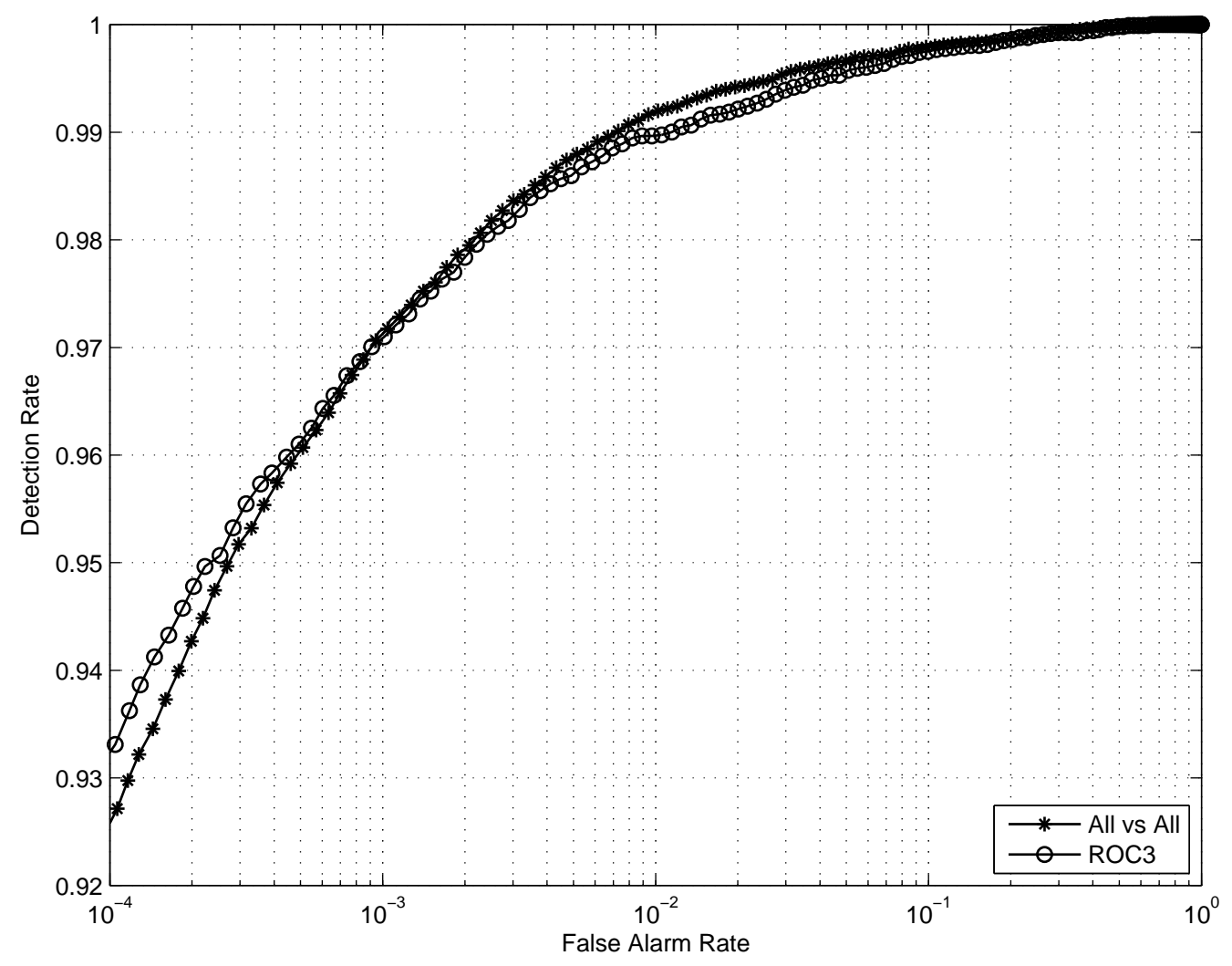

Figure 5.18: ROC curves for 5 (scales) x 4 (shape maps) x 229 (locations) x 128 (SIFT feature size) initial features (All vs All, ROC3) (UNI-CFMD)

\subsubsection{Reduced Feature Locations Experiment with 5 Selected Shape Map - Descriptor Combinations (UNI-RF-CF5D)}

We have evaluated types of shape descriptors in the previous section. One trivial observation from Figure 5.16 is that non-neutral images reduce overall performance drastically. Since non-neutral images are lacking in the training set, non-neutral recordings in test set have significant negative effects on system performance. To overcome this bottleneck, we simply removed the features, which heavily depend on expressions. Most of these features are located around the mouth and the chin. We removed 112 


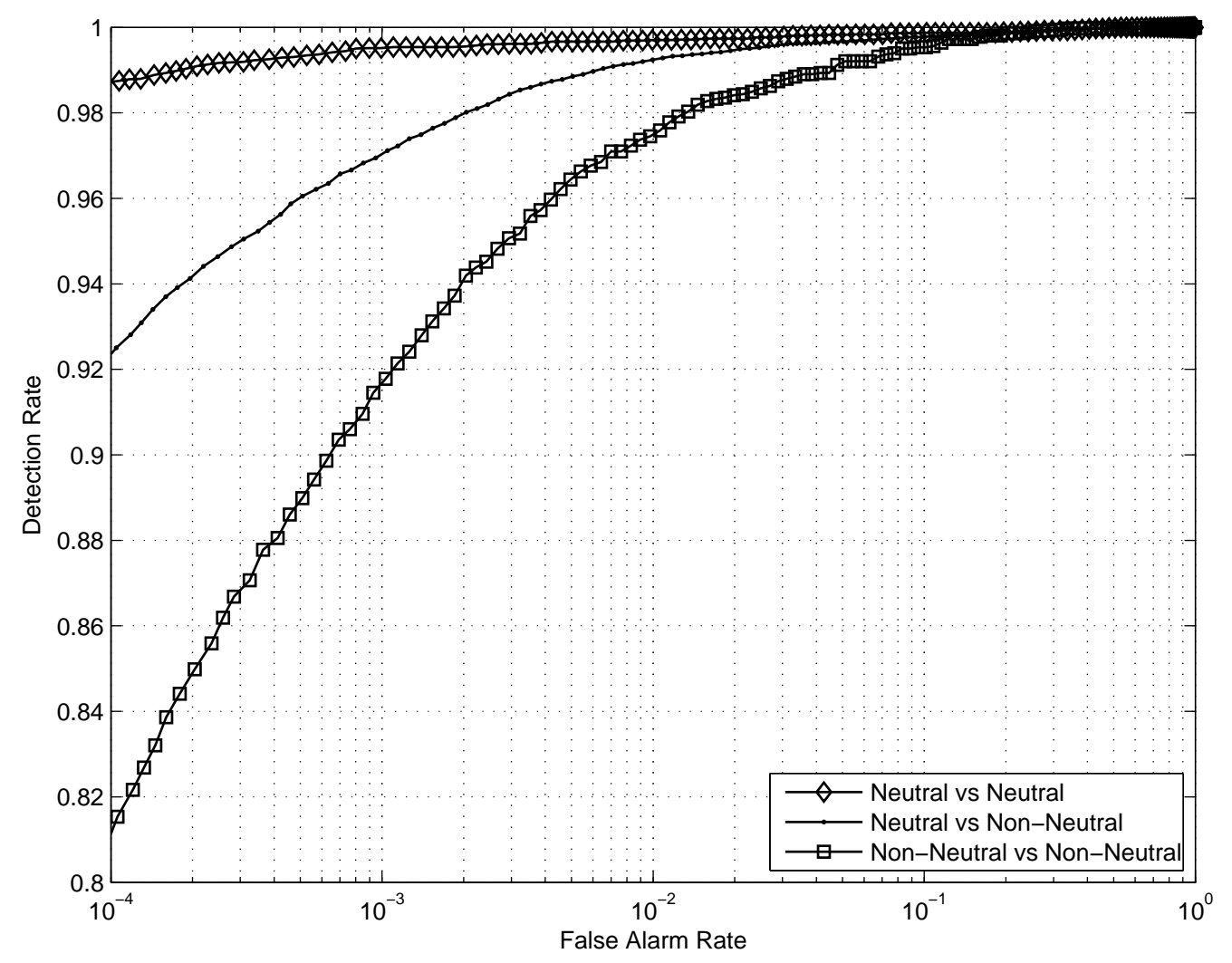

Figure 5.19: ROC curves for 5 (scales) x 4 (shape maps) x 229 (locations) x 128 (SIFT feature size) initial features(Neutral vs Neutral, Neutral vs Non-Neutral, Non-Neutral vs Non-Neutral) (UNI-CFMD) 


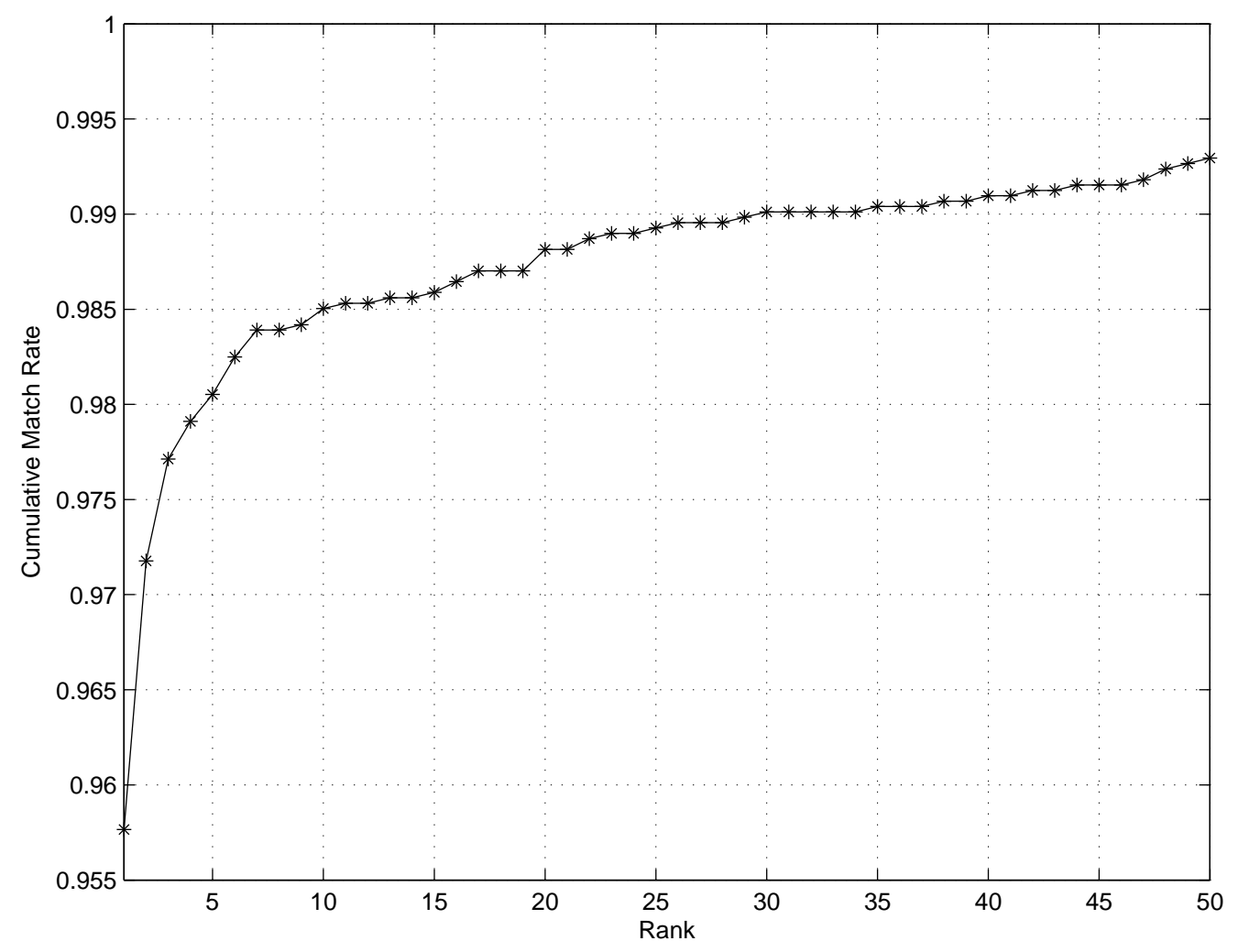

Figure 5.20: Cumulative Match Characteristics for 5 (scales) x 4 (shape maps) x 229 (locations) x 128 (SIFT feature size) initial features (UNI-CFMD) 
feature points at this area and obtained a reduced set of 345 feature points. SIFT descriptors on this reduced set of feature points is shown in Figure 5.24. In Table 5.3 , the results that are written with bold fonts correspond to the top five scores. For designing a multi-feature 3D recognition system the features in these rows are selected. A combined feature vector with a dimension of $5 \times 345 \times 128$ is generated by concatenating selected feature vectors. We performed an experiment using these combined feature vectors. This experiment has FRGC v1.0 dataset(275 subjects) as the training dataset, and FRGC v2.0 dataset(466 subjects) as the testing data. FRGC v1.0 has 275 subjects ( $n=275$ classes), therefore the LDA generates a subspace with $n-1=274$ dimensions.Receiver operating characteristics curves for this experiment are given in Figure 5.21 and Figure 5.22. In Figure 5.23, Cumulative Match Characteristics of the experiment is given. In Figure 5.21, we observe that we have obtained $98.35 \%$ detection rate at $0.1 \%$ FAR. In Figure 5.22, it can be observed that the proposed face recognition system in this study is very robust to non-neutral face scans, which is the bottleneck of all 3D face recognition systems.

The results for All vs All experiments from the recent literature are summarized in Table 5.4. To the best of our knowledge, $98.35 \%$ detection rate that we obtained at $0.1 \%$ FAR outperforms all available performances reported in the literature.

Literature summary for FRGC ROC3 experiment is given in Table 5.5. Face recognition system proposed in this study has $98.25 \%$ detection rate at $0.1 \%$ FAR which is up to the authors' knowledge again, the highest result among similar studies.

For identification, cumulative match characteristics is given in Figure 5.23. Proposed system has $97.5 \%$ rank-1 identification rate for the earliest scan as gallery, remaining as probes identification experiment. Table 5.6 represents identification results of the recent publications. The performance of our systems is $0.9 \%$ worse than the best result in the table, but still we can state that we have obtained very high identification rate for this experiment. 


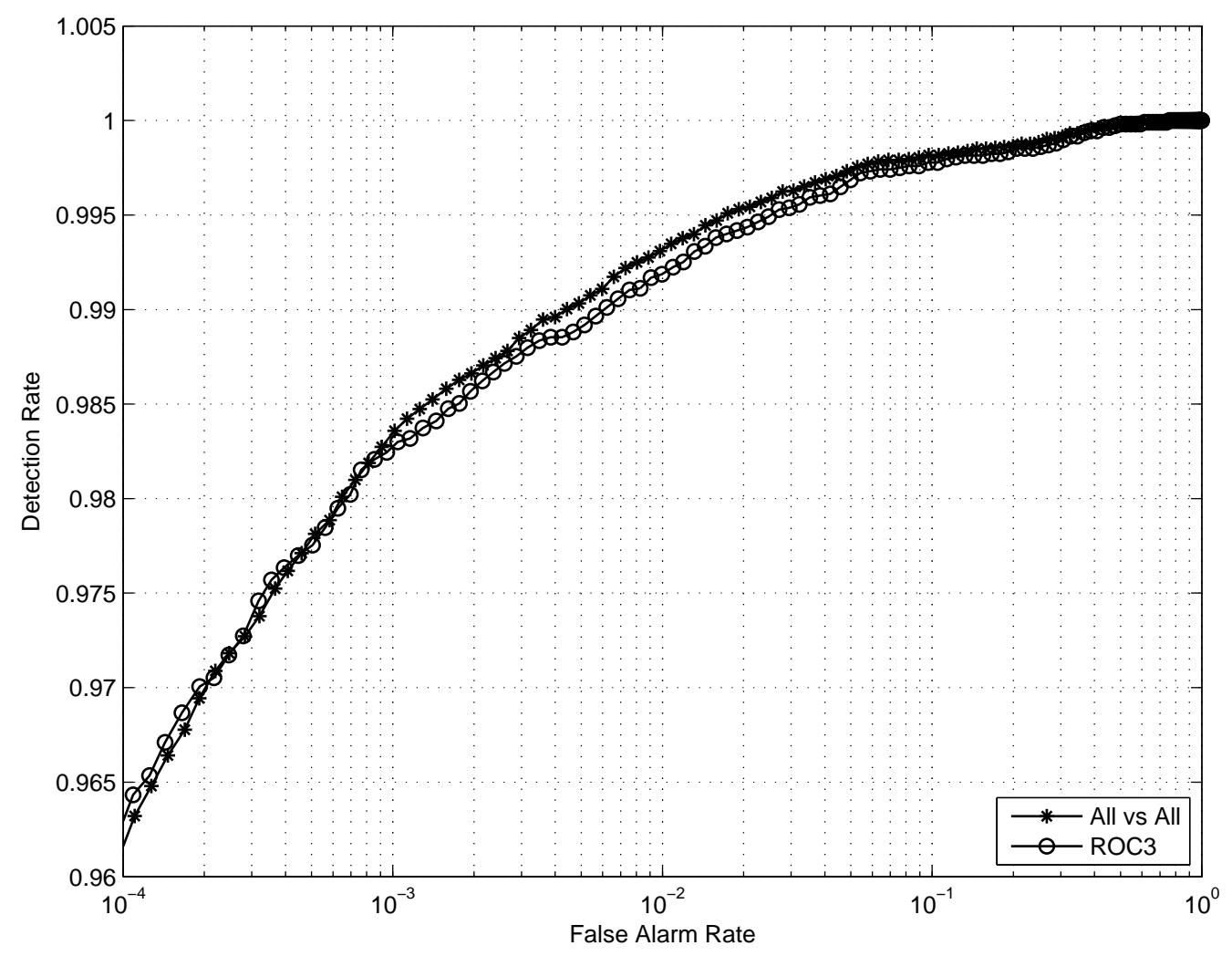

Figure 5.21: ROC curves for 5x345x128 features (All vs All, ROC3) (UNI-RFCF5D)

Table 5.4: Verification Results Using the All vs. All Data Set, at 0.1 Percent FAR

\begin{tabular}{ccc}
\hline Method & Verification Rate \% & Year \\
\hline Mian [36] & 86.6 & 2007 \\
\hline Maurer [34] & 87.0 & 2005 \\
\hline Cook [15] & 92.3 & 2006 \\
\hline Faltemier [19] & 93.2 & 2008 \\
\hline Queirolo [39] & 96.5 & 2010 \\
\hline Wang [45] & 98.13 & 2010 \\
\hline Beretti [7] & 81.2 & 2010 \\
\hline UNI-RF-CF5D & $\mathbf{9 8 . 3 5}$ & 2011 \\
\hline
\end{tabular}




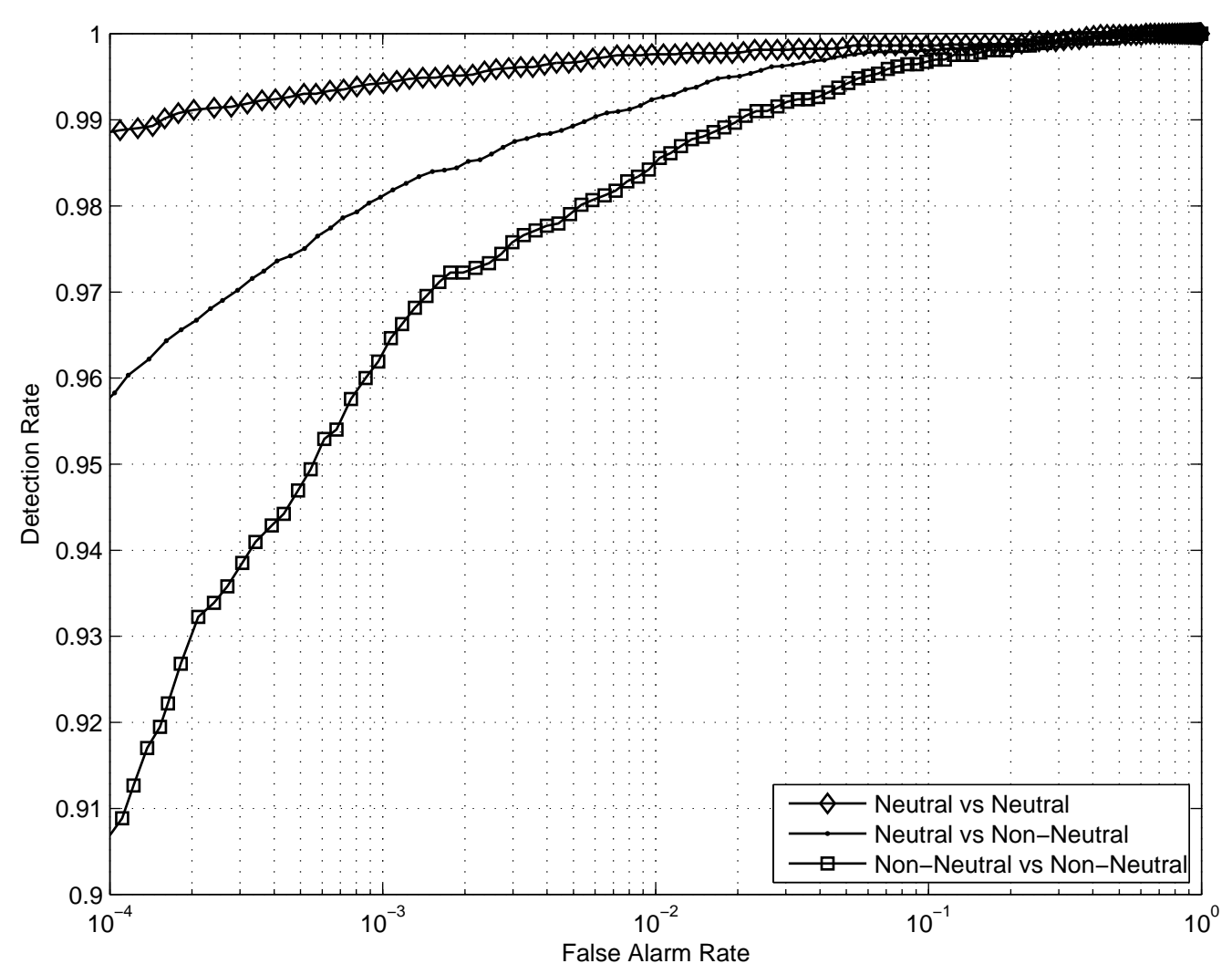

Figure 5.22: ROC curves for 5x345x128 features(Neutral vs Neutral, Neutral vs NonNeutral, Non-Neutral vs Non-Neutral) (UNI-RF-CF5D)

Table 5.5: Verification Results Using the ROC III Experiment, at 0.1 Percent FAR

\begin{tabular}{ccc}
\hline Method & Verification Rate \% & Year \\
\hline Kakadiaris [30] & 92.3 & 2006 \\
\hline Faltemier [19] & 94.8 & 2008 \\
\hline Queirolo [39] & 96.6 & 2010 \\
\hline Wang [45] & 98.04 & 2010 \\
\hline UNI-RF-CF5D & $\mathbf{9 8 . 2 5}$ & 2011 \\
\hline
\end{tabular}




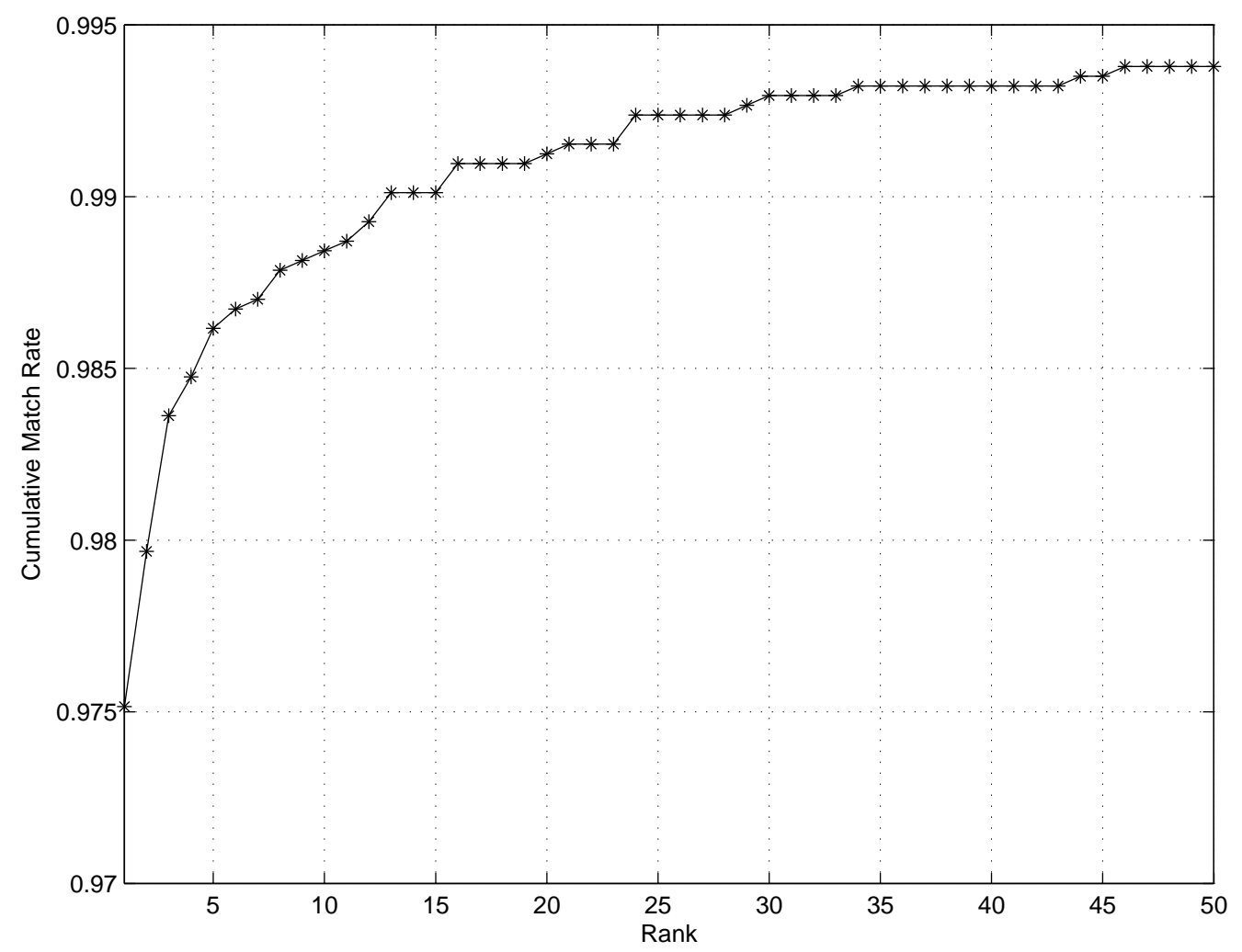

Figure 5.23: Cumulative Match Characteristics for 5x345x128 features (UNI-RFCF5D)

Table 5.6: Rank-1 Identification Results Using Earliest Scan as Gallery, Remaining as Probe

\begin{tabular}{ccc}
\hline Method & Identification Rate \% & Year \\
\hline Cook [15] & 92.9 & 2006 \\
\hline Faltemier [19] & 97.2 & 2008 \\
\hline Kakadiaris [30] & 97 & 2006 \\
\hline Wang [45] & 98.3 & 2010 \\
\hline Queirolo [39] & 98.4 & 2010 \\
\hline UNI-RF-CF5D & $\mathbf{9 7 . 5}$ & 2011 \\
\hline
\end{tabular}




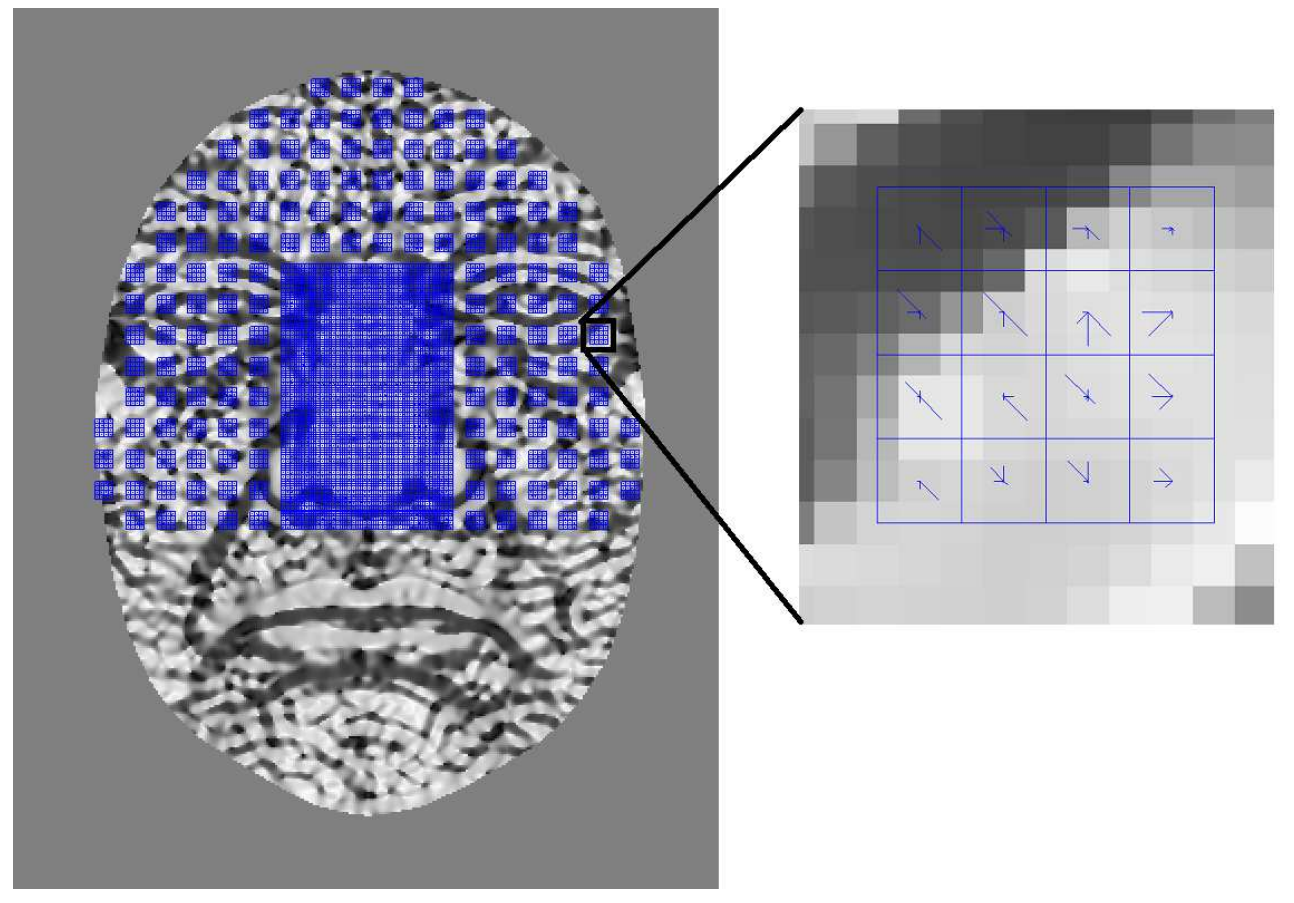

Figure 5.24: SIFT Descriptors calculated on shape index map at 345 uniformly distributed locations (mouth and chin regions excluded) 


\subsubsection{Reduced Feature Locations Experiment with Multi Shape Map- Multi Scale SIFT Descriptors (UNI-RF-CFMD)}

We generate a multi shape map - multi scale feature vector by concatenating all possible SIFT feature vectors at reduced number of feature locations. Combined feature vector has a dimension of 5 (scales) x 4(shape maps) x 345(locations) x 128(SIFT feature size). We perform an experiment using combined feature vectors. This experiment has FRGC v1.0 dataset(275 subjects) as the training dataset, and FRGC v2.0 dataset(466 subjects) as the testing data. FRGC v1.0 has 275 subjects ( $n=275$ classes), therefore the LDA generates a subspace with $n-1=274$ dimensions. Receiver operating characteristics curves for this experiment are given in Figure 5.25 and Figure 5.26. In Figure 5.27, Cumulative Match Characteristics of the experiment is given. 


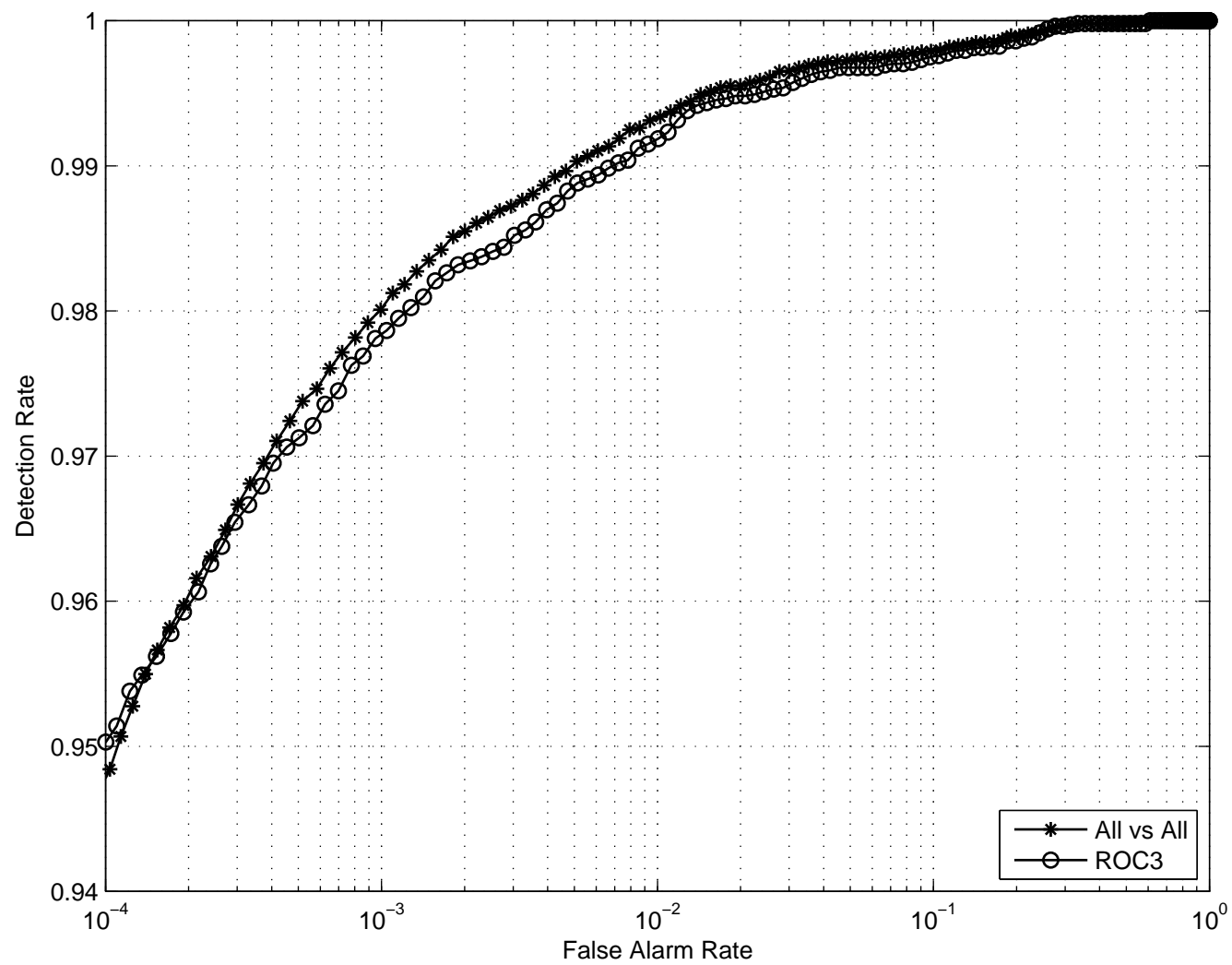

Figure 5.25: ROC curves for 5 (scales) x 4 (shape maps) x 345 (locations) x 128 (SIFT feature size) features (All vs All, ROC3) (UNI-RF-CFMD)

83 


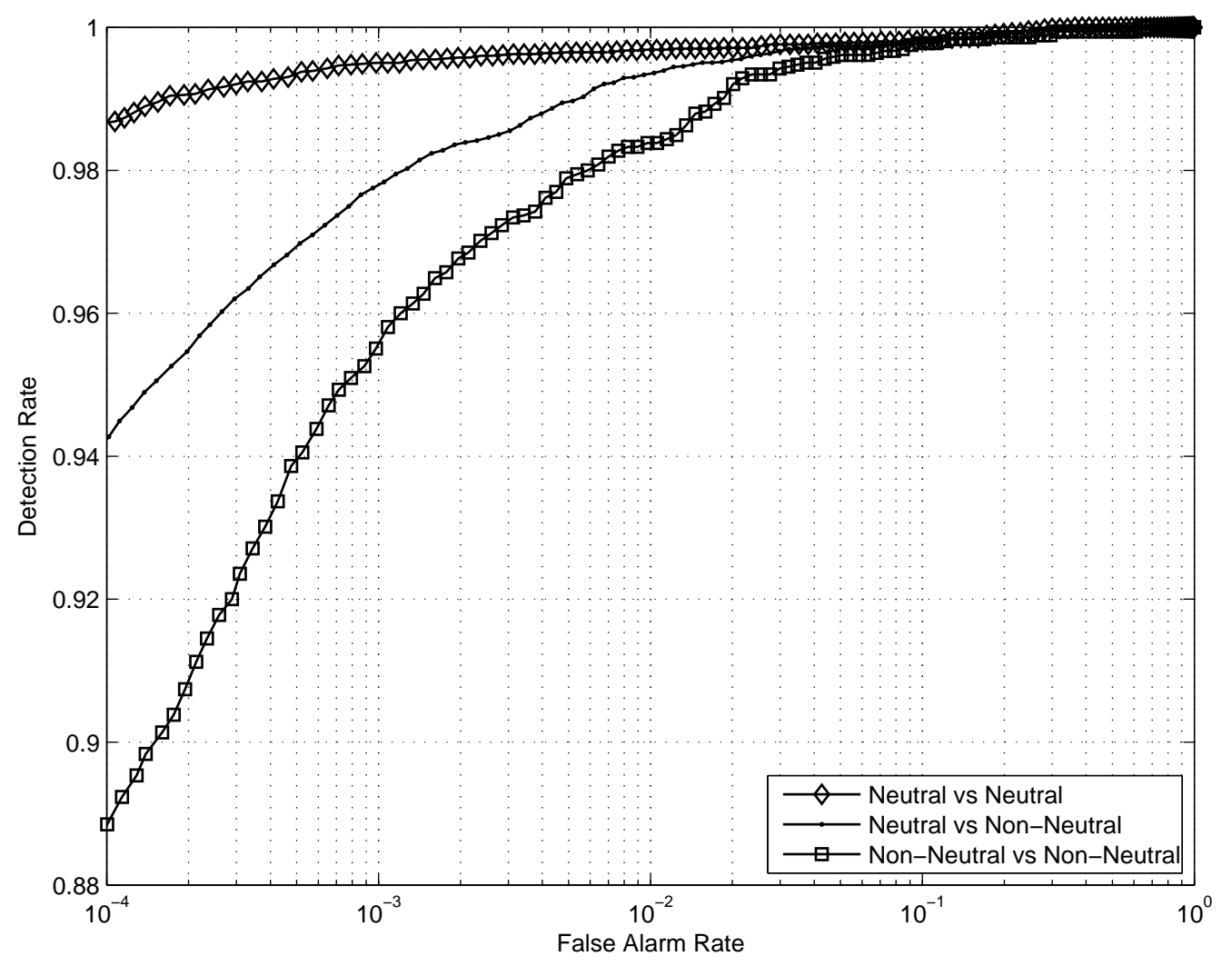

Figure 5.26: ROC curves for 5 (scales) x 4 (shape maps) x 345 (locations) x 128 (SIFT feature size) features(Neutral vs Neutral, Neutral vs Non-Neutral, Non-Neutral vs Non-Neutral ) (UNI-RF-CFMD) 


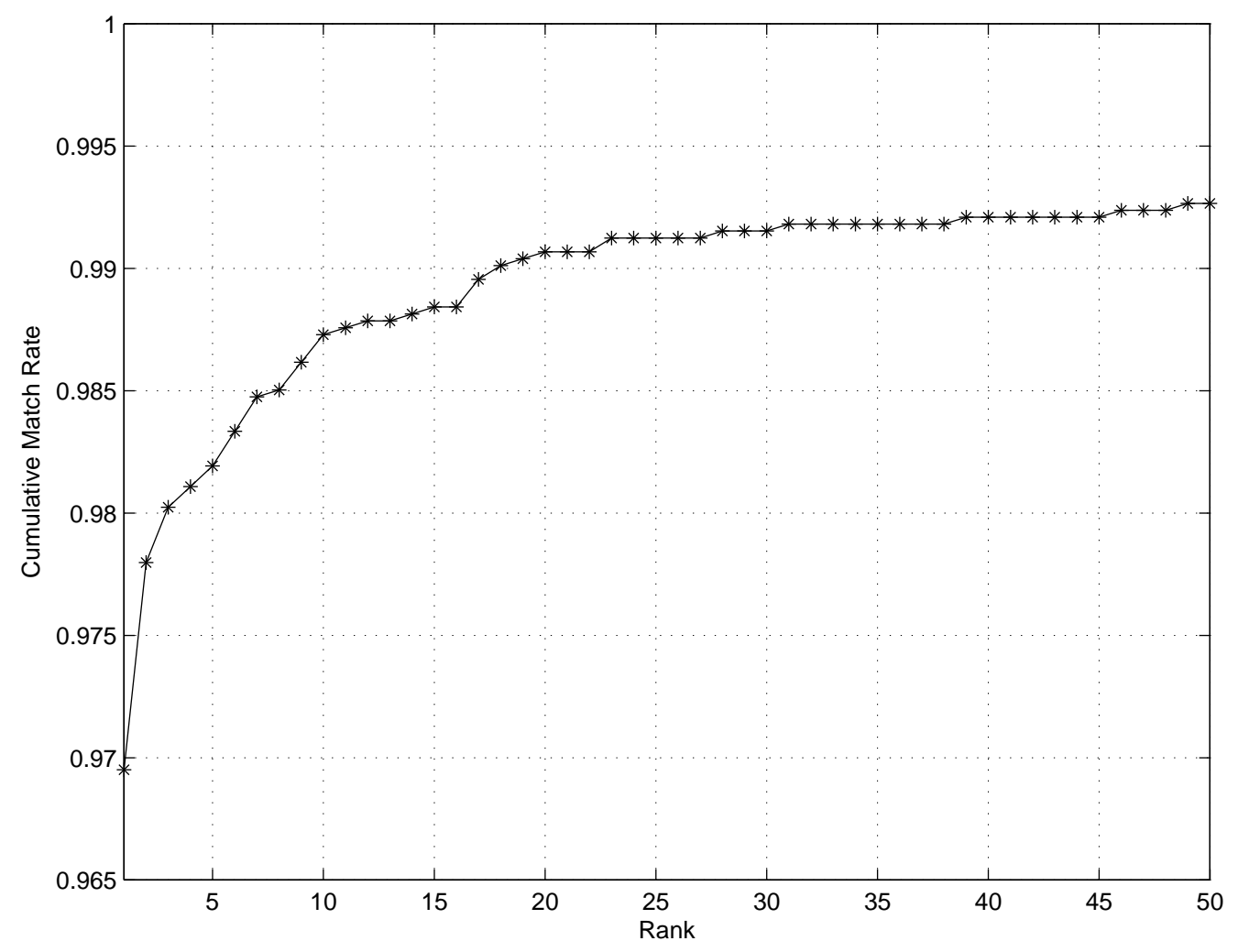

Figure 5.27: Cumulative Match Characteristics for 5 (scales) x 4 (shape maps) x 345 (locations) x 128 (SIFT feature size) initial features (UNI-RF-CFMD) 


\subsubsection{Spatial Feature Selection with Sequential Forward Selection (SFS) Algorithm}

Sequential Forward Selection (SFS) Algorithm. Feature selection is one of the essential steps of the machine learning algorithms. If the original feature set has $\mathrm{N}$ elements, then the total number of possible feature subsets is $2^{N}$. In practice, it is not possible to explore this exhaustive search space completely. A guided search can dramatically decrease the number of iterations at the cost of obtaining a sub-optimal solution. Sequential Forward Selection (SFS) Algorithm is one of the basic guided search methods. Sequential Forward Selection begins with the empty set, and in each iteration it tries to get a new feature that displays the best classifier performance with the features already selected [16]. In our case, we select the detection rate of classifier as the performance criteria.

\subsubsection{Single Region Performance on FRGCv1.0 (UNI-SR-FRGC1-11fold)}

We evaluate the discriminative power of different face regions. We divide the face into 26 regions as shown in Figure 5.28. Number of feature locations in each face region is also given in Figure 5.28. We perform experiments with each of these regions. At each time, only features those are located in a single region are used for training and testing. Shape map type is shape index, and descriptor type is SIFT with $8 \mathrm{~mm}$ width. Shape map type and descriptor type are fixed for experiments performed for each region. For all of the 26 experiments, we use the FRGC v1.0 dataset. FRGC v1.0 has 275 subjects and 250 of 275 subjects are used for training and 25 subjects are used for testing. Hence, we perform the experiments eleven times in 11-fold manner. Mean detection rates of these 26 single-region experiments are displayed in Table 5.7. Most discriminative region turn outs to be region 10 with 87,60 detection rate at $0.1 \%$ false alarm rate. 

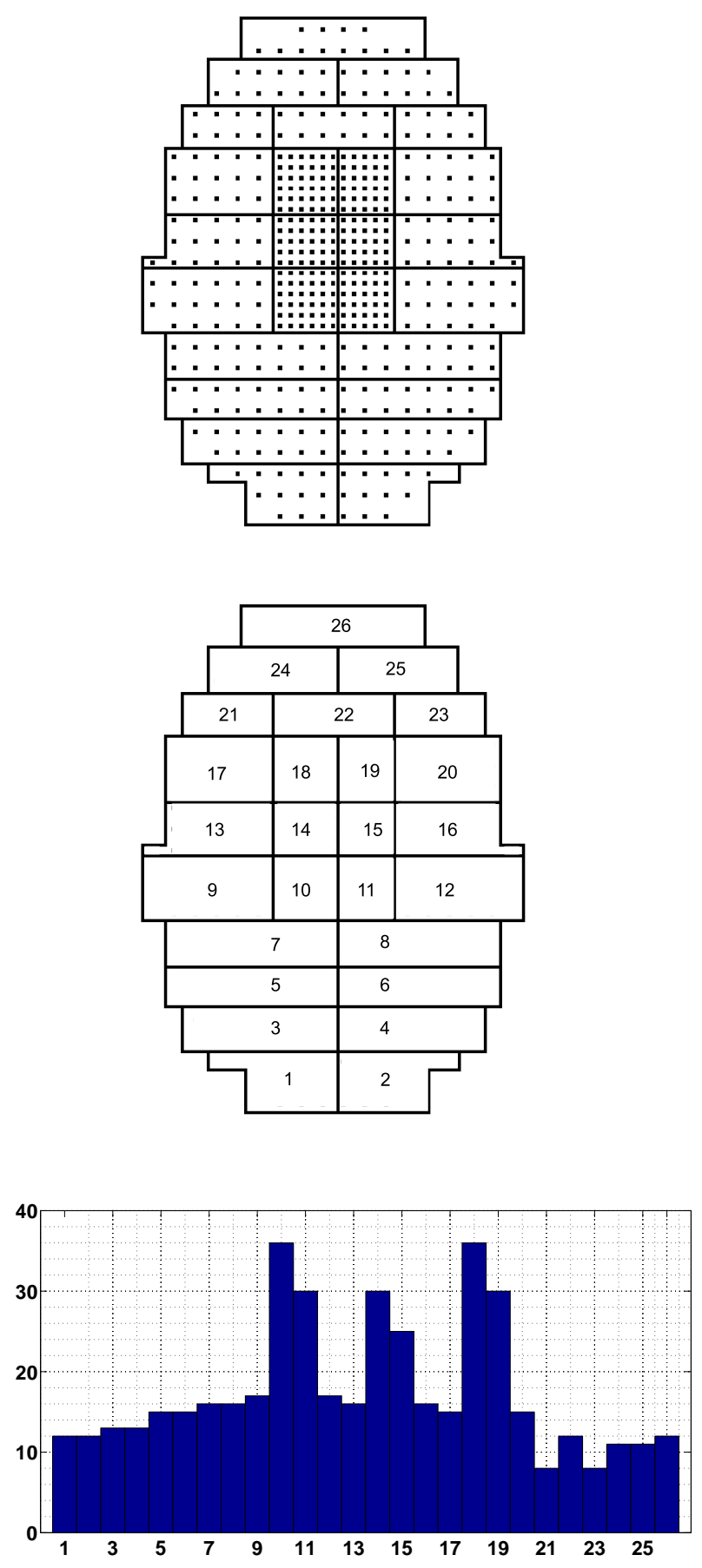

Figure 5.28: 26 Face Regions with corresponding feature locations(top), region numbers (center), number of feature locations in each face region(bottom) 
Table 5.7: Mean Detection Rates for All vs All Experiment at 0.001 False Alarm Rate with Single Face Region (Shape Index Map, SIFT Feature with 8mm width) on FRGCv1.0 Dataset (11-FOLD) (UNI-SR-FRGC1-11fold)

\begin{tabular}{cc}
\hline Region Number & DR\% \\
\hline 1 & 40,28 \\
\hline 2 & 39,21 \\
\hline 3 & 66,22 \\
\hline 4 & 65,67 \\
\hline 5 & 77,59 \\
\hline 6 & 74,09 \\
\hline 7 & 77,37 \\
\hline 8 & 78,38 \\
\hline 9 & 51,96 \\
\hline 10 & 87,60 \\
\hline 11 & 85,53 \\
\hline 12 & 53,57 \\
\hline 13 & 65,39 \\
\hline 14 & 85,20 \\
\hline 15 & 82,91 \\
\hline 16 & 64,30 \\
\hline 17 & 70,69 \\
\hline 18 & 85,77 \\
\hline 19 & 83,10 \\
\hline 20 & 71,13 \\
\hline 21 & 52,99 \\
\hline 22 & 71,70 \\
\hline 23 & 51,60 \\
\hline 24 & 35,10 \\
\hline 25 & 40,32 \\
\hline 26 & 14,19 \\
\hline &
\end{tabular}




\subsubsection{Multi Region Performance on FRGCv1.0 (UNI-SFS-FRGC1-11fold)}

Following the single-region experiments, we perform multiple region experiments as well. We start with best region and everytime we add a region with SFS. Therefore, the first experiment is done only with the region that has highest discriminative power, whereas the last experiment is done with all regions. For all of the multiple region experiments, we use the FRGC v1.0 dataset. FRGC v1.0 has 275 subjects and 250 of 275 subjects are used for training and 25 subjects are used for testing. Hence, we perform the experiments eleven times in 11-fold manner. Mean detection rates of the multiple region experiments are displayed in Table 5.8 and Figure 5.29.

Table 5.8: Mean Detection Rates for All vs All Experiment at 0.001 False Alarm Rate with Multiple Face Regions Selected by SFS Algorithm (Shape Index Map, SIFT Feature with $8 \mathrm{~mm}$ width) on FRGCv1.0 Dataset (11-FOLD) (UNI-SFS-FRGC111fold)

\begin{tabular}{cc}
\hline Region Added & DR\% \\
\hline 10 & 87,60 \\
\hline 19 & 94,67 \\
\hline 5 & 96,51 \\
\hline 25 & 97,21 \\
\hline 2 & 97,92 \\
\hline 6 & 98,39 \\
\hline 16 & 98,81 \\
\hline 22 & 99,04 \\
\hline 8 & 99,38 \\
\hline 7 & 99,38 \\
\hline 15 & 99,52 \\
\hline 1 & 99,30 \\
\hline 14 & 99,24 \\
\hline 23 & 99,48 \\
\hline 13 & 99,39 \\
\hline 3 & 99,39 \\
\hline 4 & 99,33 \\
\hline 20 & 99,16 \\
\hline 12 & 99,11 \\
\hline 24 & 99,21 \\
\hline 9 & 99,10 \\
\hline 11 & 99,08 \\
\hline 26 & 98,98 \\
\hline 21 & 98,78 \\
\hline 18 & 98,50 \\
\hline 17 & 98,32 \\
\hline &
\end{tabular}




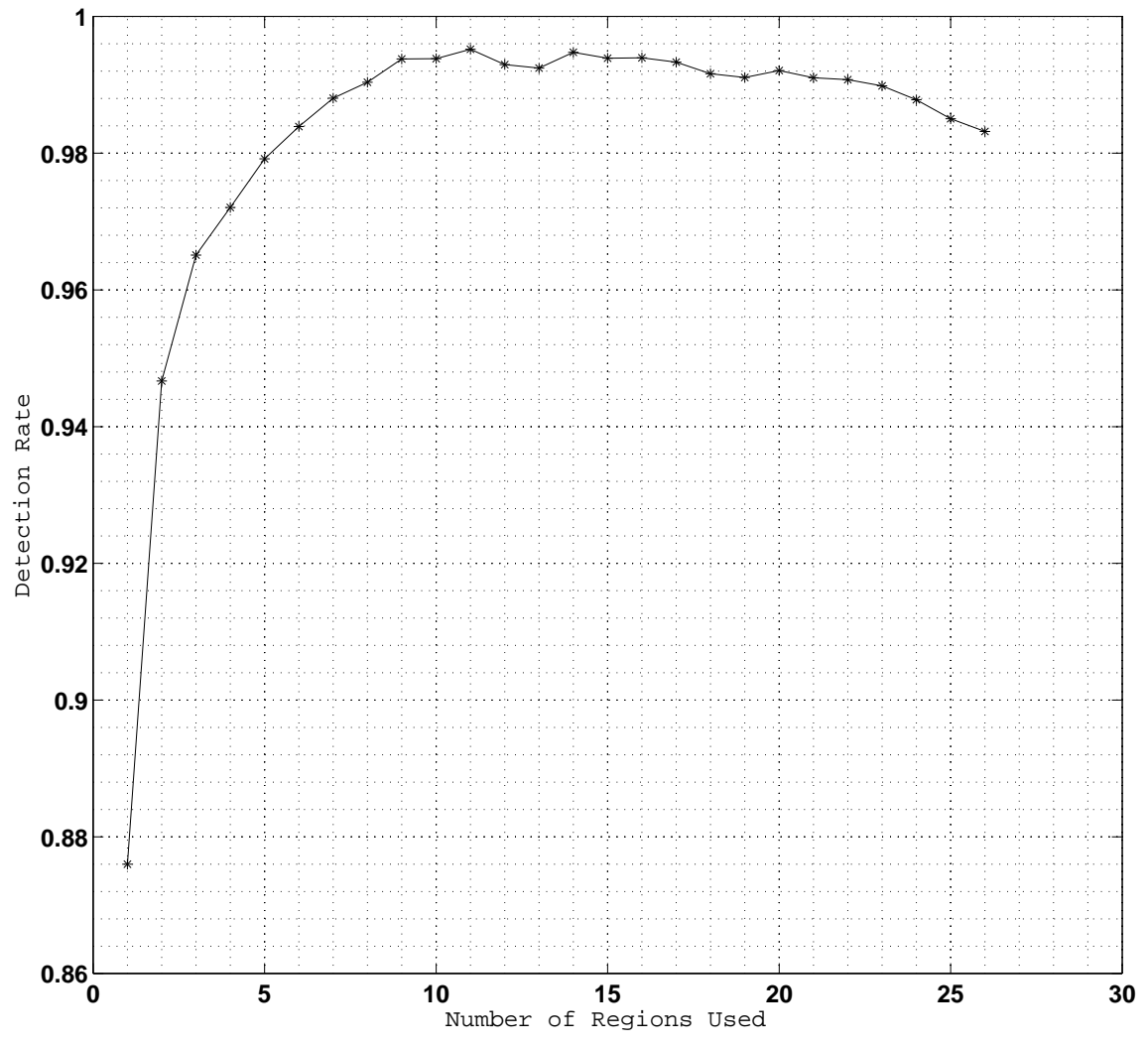

Figure 5.29: Mean Detection Rate vs Number of Regions Selected by SFS on FRGCv1.0 Dataset (11-FOLD) (UNI-SFS-FRGC1-11fold) 


\subsubsection{Single Region Performance on FRGCv2.0 (UNI-SR-FRGC2)}

We evaluate the discriminative power of different face regions also with the FRGC v2.0 database. We train each single region on FRGC v1.0 and test the performance on FRGC v2.0. Shape map type is shape index, and descriptor type is SIFT with 8 $\mathrm{mm}$ width. Shape map type and descriptor type are fixed for experiments performed for each region. Detection rates of these 26 single-region experiments are displayed in Table 5.9. Most discriminative region turn outs to be region 14 with 81,92 detection rate at $0.1 \%$ false alarm rate.

Table 5.9: Detection Rates for All vs All Experiment at 0.001 False Alarm Rate with Single Face Region (Shape Index Map, SIFT Feature with 8mm width) on FRGCv2.0 Dataset (UNI-SR-FRGC2)

\begin{tabular}{cc}
\hline Region Number & DR\% \\
\hline 1 & 19,61 \\
\hline 2 & 19,41 \\
\hline 3 & 34,15 \\
\hline 4 & 33,84 \\
\hline 5 & 39,6 \\
\hline 6 & 38,18 \\
\hline 7 & 45,13 \\
\hline 8 & 43,03 \\
\hline 9 & 46,09 \\
\hline 10 & 74,97 \\
\hline 11 & 70,97 \\
\hline 12 & 40,54 \\
\hline 13 & 60,97 \\
\hline 14 & 81,92 \\
\hline 15 & 77,82 \\
\hline 16 & 56,75 \\
\hline 17 & 63,96 \\
\hline 18 & 80,27 \\
\hline 19 & 77,7 \\
\hline 20 & 60,52 \\
\hline 21 & 43,39 \\
\hline 22 & 63,4 \\
\hline 23 & 39,66 \\
\hline 24 & 33,23 \\
\hline 25 & 32,09 \\
\hline 26 & 13,98 \\
\hline &
\end{tabular}




\subsubsection{Multi Region Performance on FRGCv2.0 (UNI-SFS-FRGC2)}

Following the single-region experiments, we perform multiple region experiments on FRGCv2.0 as well with SFS algorithm. We use FRGCv1.0 for training and FRGCv2.0 for performance feedback for SFS. Detection rates of the multiple region experiments are displayed in Table 5.10 and Figure 5.30. From Table 5.10, we observe 8 of the worst 9 regions are populated at the chin and mouth area where deformations due to expressions are large.

Table 5.10: Detection Rates for All vs All Experiment at 0.001 False Alarm Rate with Multiple Face Regions Selected by SFS Algorithm (Shape Index Map, SIFT Feature with $8 \mathrm{~mm}$ width) on FRGCv2.0 Dataset (UNI-SFS-FRGC2)

\begin{tabular}{cc}
\hline Region Added & DR\% \\
\hline 14 & 81,92 \\
\hline 22 & 91,01 \\
\hline 16 & 93,37 \\
\hline 17 & 94,41 \\
\hline 10 & 95,33 \\
\hline 25 & 95,89 \\
\hline 19 & 96,13 \\
\hline 20 & 96,35 \\
\hline 9 & 96,53 \\
\hline 15 & 96,67 \\
\hline 26 & 96,75 \\
\hline 12 & 96,79 \\
\hline 18 & 96,94 \\
\hline 23 & 96,98 \\
\hline 11 & 96,96 \\
\hline 13 & 96,98 \\
\hline 21 & 97,06 \\
\hline 1 & 97,07 \\
\hline 24 & 97,02 \\
\hline 5 & 96,98 \\
\hline 2 & 96,9 \\
\hline 8 & 96,69 \\
\hline 4 & 96,51 \\
\hline 3 & 96,23 \\
\hline 7 & 96,02 \\
\hline 6 & 95,78 \\
\hline &
\end{tabular}




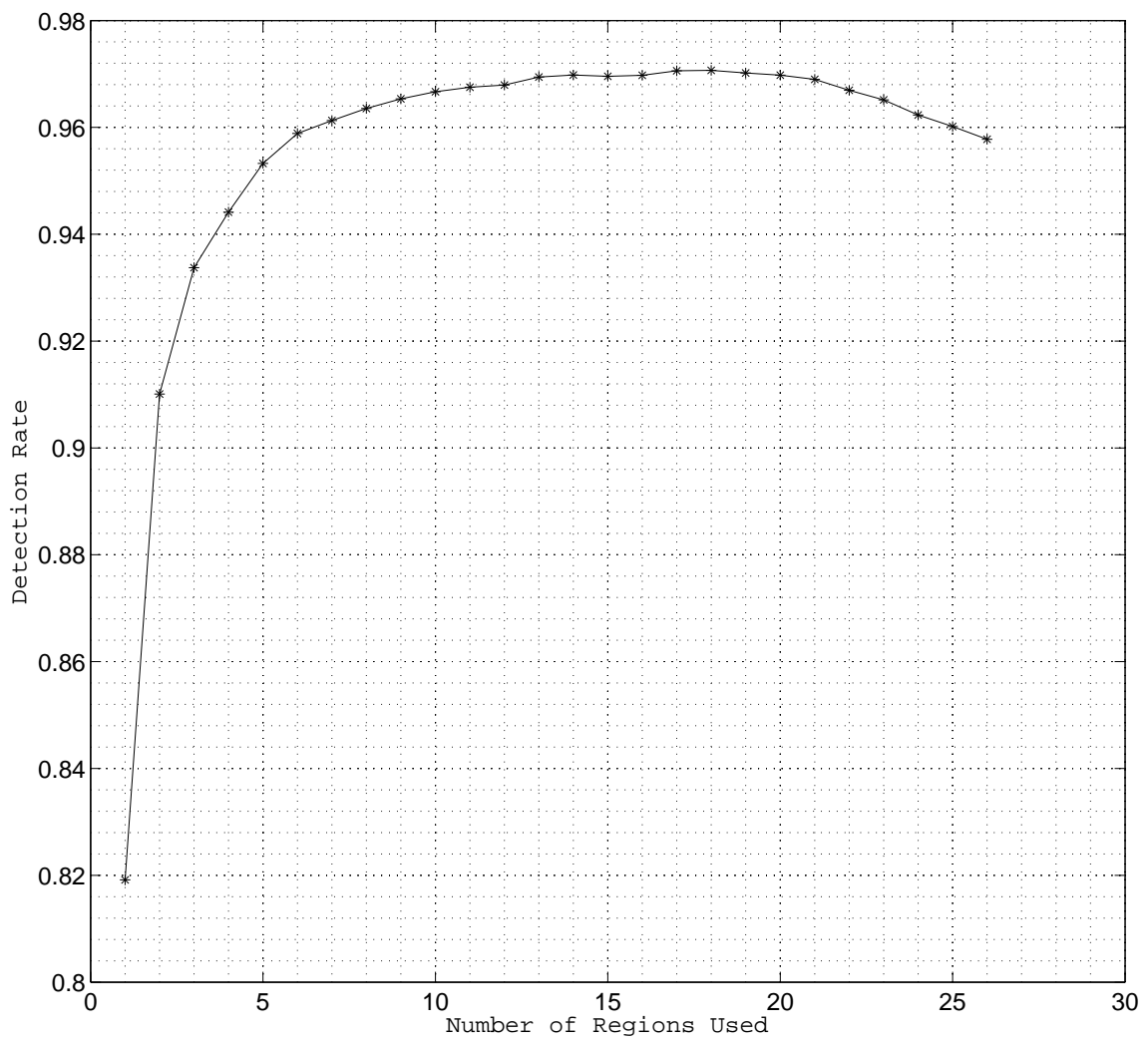

Figure 5.30: Detection Rate vs Number of Regions Selected by SFS on FRGCv2.0 Dataset (UNI-SFS-FRGC2) 


\subsection{Experimental Results for Generic Model Supported 3D Face Recognition}

Experiments are made with the FRGC database to test the performance of the generic model supported face recognition system. We have used 941 images (2 images are not annotated) from FRGCv1.0 for training and 3980 images (27 images are not annotated) from FRGCv2.0 for testing. FRGC v1.0 has 275 subjects ( $\mathrm{n}=275$ classes), therefore the LDA generates a subspace with $n-1=274$ dimensions.

\subsubsection{Evaluation of different shape maps and descriptor sizes (GEN-EVA)}

We performed recognition experiments with different size SIFT descriptors. Descriptor sizes used throughout the experiments were 4, 8, 12, 16, 32 millimeters. For the SURF descriptors, gaussian variance, $\sigma$, had the values of $1,2,3,4,5$ millimeters representing different scales. Smaller sized descriptors focus more on local variations, whereas larger scales correspond to global shape variations.

The experimental results for All vs All experiment, are given in Table 5.11. Detection rates for All vs All experiment at 0.001 false alarm rate are reported for different descriptor sizes and different shape maps.

The experiment presented at third row of Table 5.11 has the best result among others. For this experiment we obtain $93.62 \%$ detection rate at $0.1 \%$. Receiver operating characteristics curves of third row experiment are given in Figure 5.31, Figure 5.32, Figure 5.33, Figure 5.34 and Figure 5.35. We can state that a mid-scaled SIFT descriptor applied on shape index map has more discriminative power for the problem of face recognition. 
Table 5.11: Detection Rates at 0.001 False Alarm Rate of Generic Model Supported 3D Face Recognition System for Different Sized Local Descriptors and Different Shape Maps (GEN-EVA)

\begin{tabular}{|c|c|c|c|c|}
\hline Descriptor & Shape Map & Size $(\mathrm{mm})$ & Dimension & DR\% \\
\hline SIFT & SI & 4 & $128 \times 549$ & 77,21 \\
\hline SIFT & $\mathrm{SI}$ & 8 & $128 \times 549$ & 91,78 \\
\hline SIFT & SI & 12 & $128 \times 549$ & 93,62 \\
\hline SIFT & SI & 16 & $128 \times 549$ & 93,06 \\
\hline $\begin{array}{l}\text { SIFT } \\
\end{array}$ & SI & 32 & $128 \times 549$ & 84,26 \\
\hline SIFT & $\mathrm{C}$ & 4 & $128 \times 549$ & 76,87 \\
\hline SIFT & $\mathrm{C}$ & 8 & $128 \times 549$ & 82,89 \\
\hline SIFT & $\mathrm{C}$ & 12 & $128 \times 549$ & 79,46 \\
\hline SIFT & $\mathrm{C}$ & 16 & $128 \times 549$ & 76,23 \\
\hline SIFT & $\mathrm{C}$ & 32 & $128 \times 549$ & 56,81 \\
\hline SIFT & $\mathrm{H}$ & 4 & $128 \times 549$ & 76,23 \\
\hline SIFT & $\mathrm{H}$ & 8 & $128 \times 549$ & 88,58 \\
\hline SIFT & $\mathrm{H}$ & 12 & $128 \times 549$ & 88,09 \\
\hline SIFT & $\mathrm{H}$ & 16 & $128 \times 549$ & 87,27 \\
\hline SIFT & $\mathrm{H}$ & 32 & $128 \times 549$ & 54,48 \\
\hline SIFT & $\mathrm{K}$ & 4 & $128 \times 549$ & 68,13 \\
\hline SIFT & $\mathrm{K}$ & 8 & $128 \times 549$ & 82,02 \\
\hline SIFT & $\mathrm{K}$ & 12 & $128 \times 549$ & 82,26 \\
\hline $\begin{array}{l}\text { SIFT } \\
\end{array}$ & $\bar{K}$ & 16 & $128 \times 549$ & 81,51 \\
\hline SIFT & $\mathrm{K}$ & 32 & $128 \times 549$ & 49,07 \\
\hline SURF & SI & 1 & $64 \times 549$ & 60,33 \\
\hline$\overline{\text { SURF }}$ & SI & 2 & $64 \times 549$ & 82,46 \\
\hline SURF & SI & 3 & $64 \times 549$ & 87,18 \\
\hline SURF & SI & 4 & $64 \times 549$ & 87,52 \\
\hline SURF & SI & 5 & $64 \times 549$ & 87,79 \\
\hline SURF & $\mathrm{C}$ & 1 & $64 \times 549$ & 60,39 \\
\hline SURF & $\mathrm{C}$ & 2 & $64 \times 549$ & 72,93 \\
\hline SURF & $\mathrm{C}$ & 3 & $64 \times 549$ & 73,05 \\
\hline SURF & $\mathrm{C}$ & 4 & $64 \times 549$ & 71,12 \\
\hline SURF & $\mathrm{C}$ & 5 & $64 \times 549$ & 69,98 \\
\hline SURF & $\mathrm{H}$ & 1 & $64 \times 549$ & 57,61 \\
\hline SURF & $\overline{\mathrm{H}}$ & 2 & $64 \times 549$ & 73,15 \\
\hline SURF & $\mathrm{H}$ & 3 & $64 \times 549$ & 74,21 \\
\hline SURF & $\mathrm{H}$ & 4 & $64 \times 549$ & 73,11 \\
\hline SURF & $\mathrm{H}$ & 5 & $64 \times 549$ & 71,76 \\
\hline SURF & $\mathrm{K}$ & 1 & $64 \times 549$ & 51,4 \\
\hline SURF & $\mathrm{K}$ & 2 & $64 \times 549$ & 60,53 \\
\hline SURF & $\bar{K}$ & 3 & $64 \times 549$ & 58,87 \\
\hline SURF & $\mathrm{K}$ & 4 & $64 \times 549$ & 55,56 \\
\hline SURF & $\mathrm{K}$ & 5 & $64 \times 549$ & 53,08 \\
\hline
\end{tabular}




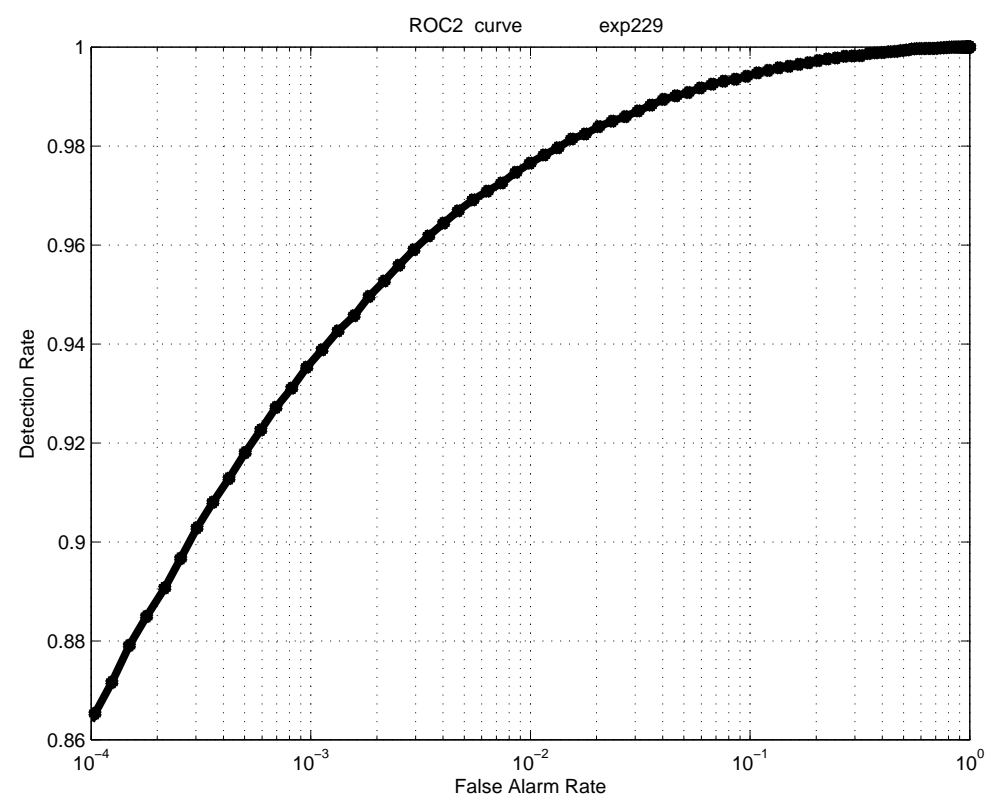

Figure 5.31: ROC curve for 549x128 features on generic model (All vs All) (GENEVA)

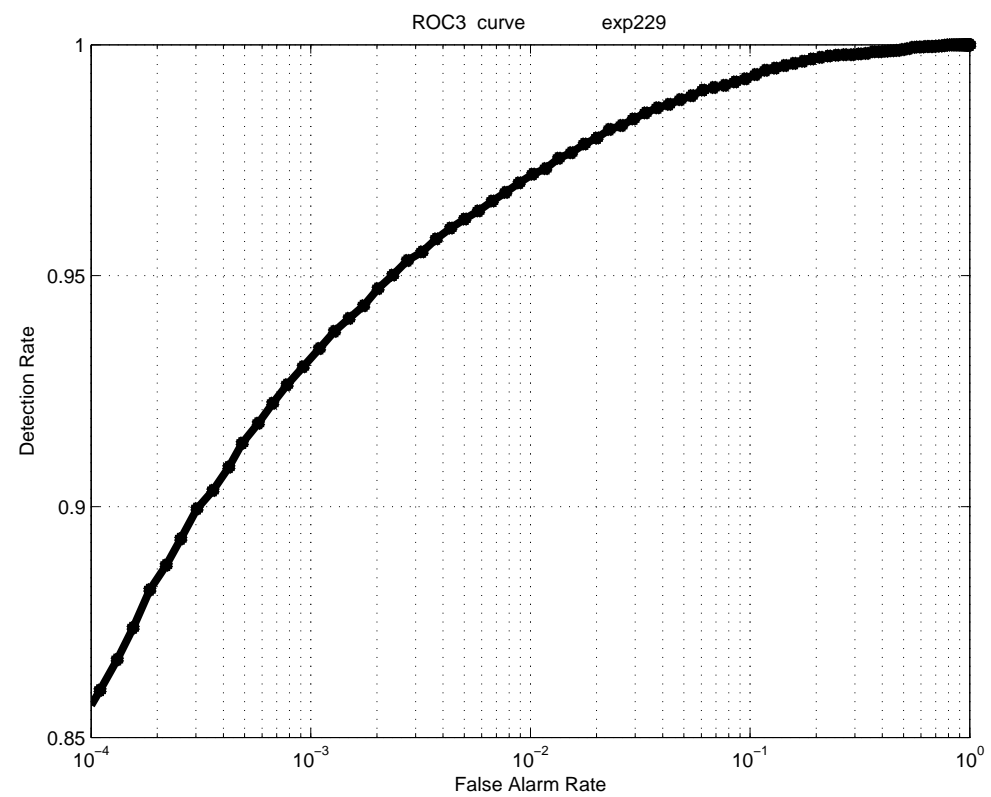

Figure 5.32: ROC curve for $549 \times 128$ features on generic model (Fall2003 vs Spring2004) (GEN-EVA)

96 


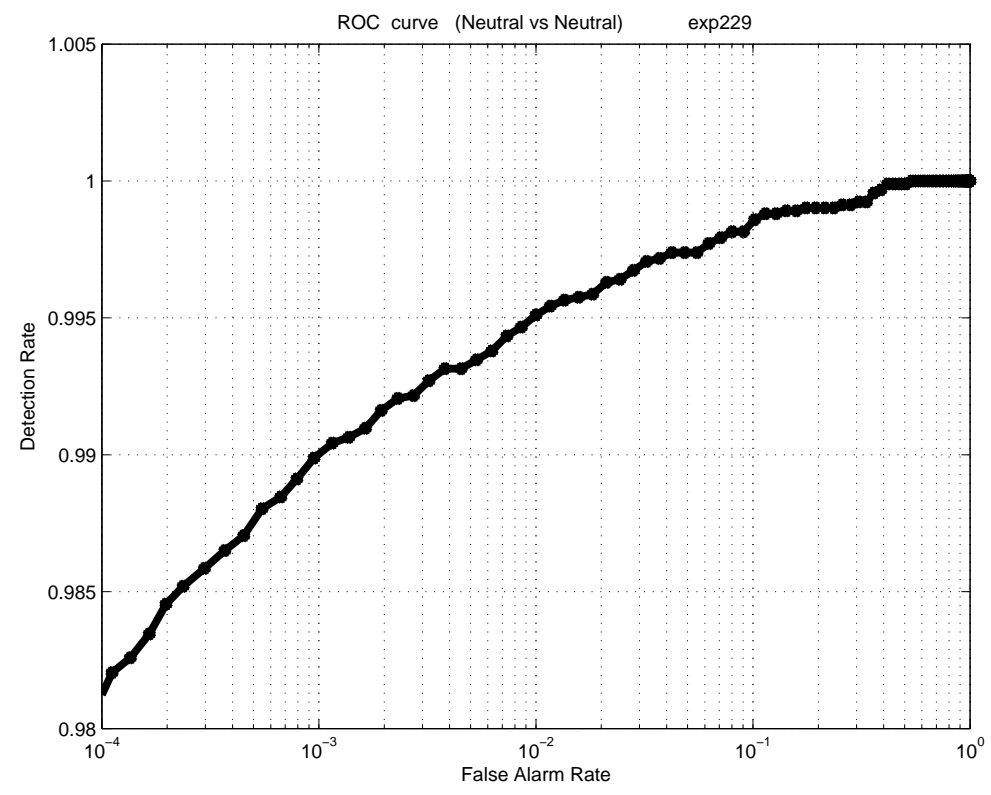

Figure 5.33: ROC curve for 549x128 features on generic model (Neutral vs Neutral) (GEN-EVA)

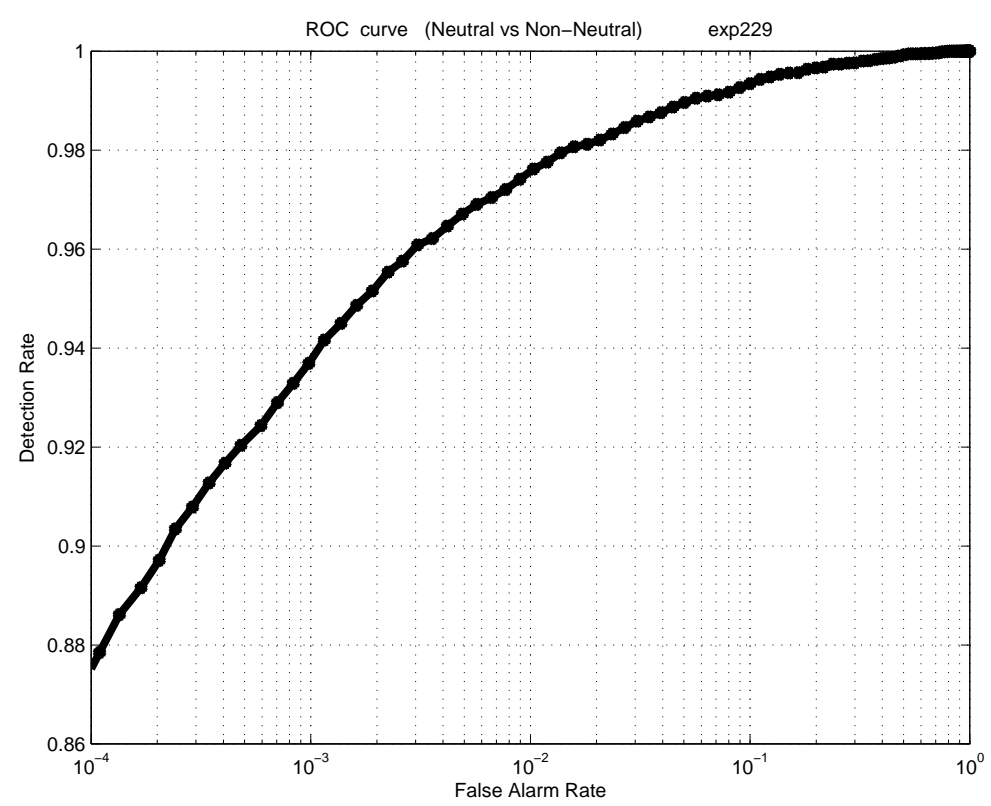

Figure 5.34: ROC curve for 549x128 features on generic model (Neutral vs NonNeutral) (GEN-EVA)

97 


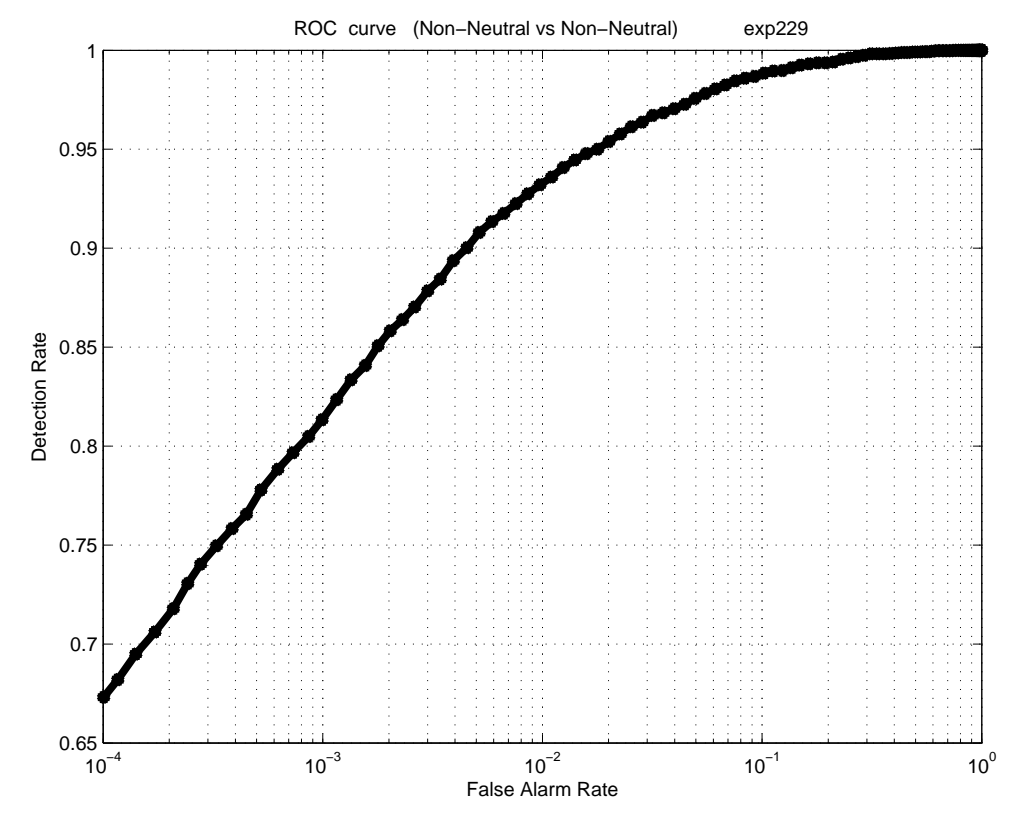

Figure 5.35: ROC curve for 549x128 features on generic model (Non-Neutral vs NonNeutral) (GEN-EVA) 


\section{CHAPTER 6}

\section{CONCLUSION}

\subsection{Summary}

In this study, we have proposed two types of 3D face recognition methods. The first method we proposed is a full automatic one, which works with local shape descriptors that are uniformly distributed across the face. We initialize the face recognition process by preprocessing the original 3D face scans. Preprocessing includes noise removal, smoothing and robust 3D registration. After the preprocessing stage, differential shape maps (shape index, curvedness, mean curvature and gaussian curvature) are calculated using the range data. Hence we get 2D differential shape maps involving rich 3D geometry information. In the next stage, SIFT and SURF descriptors are calculated at uniformly distributed locations at fixed scale and orientation. These shape descriptor vectors are used in a LDA classifier. The decision for verification/identification is made by comparing the vectors with reduced dimensionality in the subspace obtained by LDA. The performance of the proposed 3D face recognition system is tested in the FRGC database.

The second method uses generic face modeling and local shape descriptors calculated at the nodes of the generic face mesh. Generic face model is initiated by manual annotation of 50 landmarks on the face. Then, generic face model is deformed to fit the $3 \mathrm{D}$ face scan. Local shape descriptors are calculated at the nodes of the generic mesh and fed into a LDA classifier. A discriminative subspace with reduced number of dimensions is obtained. Face comparisons are made in this low dimensional subspace and their results are reported in accordance with the FRGC experimental protocol. 
Up to our knowledge, we have applied local shape descriptors (shape index + SIFT, mean curvature + SIFT,$\ldots)$ with LDA subspace projection for the 3D face recognition problem successfully for the first time. The calculation of the local shape descriptors does not have much computational cost. By using LDA subspace projection, we can reduce the number of dimensions from ten thousands to hundreds, therefore this reduction drastically reduces face comparison time in the final stage. 3D face recognition system we have proposed has a competitive performance with the results reported in the recent literature at a low computational cost.

\subsection{Discussion}

Our approach uses edge histograms of local differential geometry parameters as local shape descriptors. Discriminative part of these shape descriptors are obtained by linear discriminant analysis (LDA) and similarity score is generated by comparing the target and query feature vectors, which are in the reduced subspace. The proposed method has been tested on the FRGC v2.0 database. We achieved $98.35 \%$ and $98.25 \%$ detection rates at $0.1 \%$ false alarm rate for All vs. All and ROC3 experiments respectively. Rank-1 identification rate for earliest scan as gallery and the remaining scans as probes experiment is $97.5 \%$. To the best of our knowledge, our verification rates are the best results among similar studies in $3 \mathrm{D}$ face recognition literature. Our rank-1 identification rate is also very high for FRGC v2.0 database. A number of factors increased the performance of the proposed system. First factor is using a fixed dense grid for the feature locations instead of keypoint matching and so getting the maximum shape information. Second factor is removing the feature locations around the chin and mouth, shapes of which are easiliy affected by expresions. Thirdly, we have observed that application of cosine similarity metric in the LDA subspace increases detection and recogntion performance. Last, but not the least factor is feature level fusion of different types of shape descriptors. Generic model supported 3D face recognition system shows significant performance on the FRGC database as well. We achieved $93.62 \%$ detection rate at $0.1 \%$ false alarm rate for All vs. All ecperiment with a single type of descriptor. Results can be further improved by better generic model selection. A better generic face model used for recognition purposes should 
have finer grid resolution where face has more discriminative power.

The SIFT descriptor is used in the recent literature for both $2 \mathrm{D}[17,21]$ and $3 \mathrm{D}$ [25, 48] face recognition. The methods in [25] and [48] apply SIFT descriptors on shape index map similar to the method we have proposed. However both studies, like the similar ones in the literature, focus on the keypoint matching process. Instead, we use fixed location and fixed scale descriptors by the support of global registration. Furthermore, we do not prefer direct matching of SIFT descriptors, rather we use LDA to obtain a discriminative subspace projection and the similarity score is calculated in the subspace with reduced dimensionality. We should note that similarity score generation in the reduced dimensionality subspace is a great advantage especially for the large databases.

\subsection{Future Work}

In this study, we evaluated the performance of the local shape descriptor supported 3D face recognition system. We performed experiments on the FRGCv2.0 database, which includes 3D face scans with expressions up to a moderate level. The high system performance we have achieved for this particular database should be inspected by using another database, which has 3D face scans with stronger expressions.

In the preprocessing stage of our 3D face recognition system, we applied pose and orientation correction based on the shape map symmetry of the 3D face scan. This method served as a valid pose and orientation normalization step for the FRGC database in which subjects are instructed to look directly at the face scanner. It is known that there are face databases, which have large pan, tilt, and roll variations. For such 3D face scans with larger orientation variations, a more robust preprocessing stage should be developed.

LDA on SIFT histograms turned out to be a successful tool in the 3D face recognition domain. We have strong experimental clues supporting the usefulness of LDA on SIFT. As a future work, we hope to improve this tool exploring the theoretical aspects of optimal linear combinations of histograms for a general classification problem. 


\section{REFERENCES}

[1] Facegen 3d human faces,http://www.facegen.com/, last visited on 13/08/2011.

[2] a Abate, M Nappi, D Riccio, and G Sabatino. 2D and 3D face recognition: A survey. Pattern Recognition Letters, 28(14):1885-1906, October 2007.

[3] Nese Alyuz, Berk Gokberk, and Lale Akarun. Regional Registration for Expression Resistant 3-D Face Recognition. IEEE Transactions on Information Forensics and Security, 5(3):425-440, September 2010.

[4] Herbert Bay, Andreas Ess, Tinne Tuytelaars, and Luc Van Gool. Speeded-up robust features (surf). Comput. Vis. Image Underst., 110:346-359, June 2008.

[5] Neslihan Bayramoglu and A. Aydin Alatan. Shape index sift: Range image recognition using local features. Pattern Recognition, International Conference on, $0: 352-355,2010$.

[6] R. Bellman. Adaptive control processes: a guided tour. Princeton University Press, Princeton, New Jersey, USA, 19:94, 1961.

[7] Stefano Berretti, Alberto Del Bimbo, and Pietro Pala. 3d face recognition using isogeodesic stripes. IEEE Transactions on Pattern Analysis and Machine Intelligence, 32:2162-2177, 2010.

[8] Charles Beumier and Marc Acheroy. Face verification from 3d and grey level clues. Pattern Recognition Letters, 22(12):1321 - 1329, 2001. Selected Papers from the 11th Portuguese Conference on Pattern Recognition - RECPAD2000.

[9] Kevin W. Bowyer, Kyong Chang, and Patrick J. Flynn. A survey of approaches and challenges in $3 \mathrm{D}$ and multi-modal $3 \mathrm{D}+2 \mathrm{D}$ face recognition. Computer Vision and Image Understanding, 101(1):1-15, 2006.

[10] K.W. Bowyer, K.I. Chang, P.J. Flynn, and Xin Chen. Face recognition using 2-d, 3-d, and infrared: Is multimodal better than multisample? Proceedings of the IEEE, 94(11):2000 -2012, nov. 2006.

[11] Alexander M. Bronstein, Michael M. Bronstein, and Ron Kimmel. Threedimensional face recognition. Int. J. Comput. Vision, 64:5-30, August 2005.

[12] Deng Cai. Spectral Regression: A Regression Framework for Efficient Regularized Subspace Learning. PhD thesis, Department of Computer Science, University of Illinois at Urbana-Champaign, May 2009.

[13] K. I. Chang, K. W. Bowyer, and P. J. Flynn. Multiple nose region matching for 3D face recognition under varying facial expression. IEEE Trans. Pattern Analysis and Machine Intelligence, 28(10):1695-1700, October 2006. 
[14] Jamie Cook, Vinod Ch, and Clinton Fookes. 3d face recognition using loggabor templates. In in Proc. British Machine Vision Conf., 2006, 2006.

[15] Jamie Cook, Chris McCool, Vinod Chandran, and Sridha Sridharan. Combined 2d/3d face recognition using log-gabor templates. Advanced Video and Signal Based Surveillance, IEEE Conference on, 0:83, 2006.

[16] M. Dash and H. Liu. Feature Selection for Classification. Intelligent Data Analysis, 1:131-156, 1997.

[17] O. Déniz, G. Bueno, and J. Salido[a] F. De La Torre. Face Recognition using Histograms of Oriented Gradients. Pattern Recognition Letters, 32(12):1598 1603, January 2011.

[18] T.C. Faltemier, K.W. Bowyer, and P.J. Flynn. Using multi-instance enrollment to improve performance of 3D face recognition. Computer Vision and Image Understanding, 112(2):114-125, 2008.

[19] Timothy C. Faltemier, Kevin W. Bowyer, and Patrick J. Flynn. A Region Ensemble for 3-D Face Recognition. IEEE Transactions on Information Forensics and Security, 3(1):62-73, 2008.

[20] R. A. Fisher. The use of multiple measurements in taxonomic problems. Annals of Eugenics, 7(7):179-188, 1936.

[21] Cong Geng and Xudong Jiang. Face recognition based on the multi-scale local image structures. Pattern Recognition, March 2011.

[22] Berk Gökberk, Helin Dutağacı, Aydin Ulas, Lale Akarun, and Bülent Sankur. Representation plurality and fusion for 3-D face recognition. IEEE transactions on systems, man, and cybernetics. Part B, Cybernetics : a publication of the IEEE Systems, Man, and Cybernetics Society, 38(1):155-173, February 2008.

[23] T Heseltine, N Pears, and J Austin. Three-dimensional face recognition using combinations of surface feature map subspace components. Image and Vision Computing, 26(3):382-396, March 2008.

[24] C. Hesher, A. Srivastava, and G. Erlebacher. A novel technique for face recognition using range imaging. In Signal Processing and Its Applications, 2003. Proceedings. Seventh International Symposium on, volume 2, pages 201 - 204 vol.2, july 2003.

[25] Di Huang, Guangpeng Zhang, M. Ardabilian, Yunhong Wang, and Liming Chen. $3 \mathrm{~d}$ face recognition using distinctiveness enhanced facial representations and local feature hybrid matching. In Biometrics: Theory Applications and Systems (BTAS), 2010 Fourth IEEE International Conference on, pages 1-7, sept. 2010.

[26] Michael Husken, Michael Brauckmann, Stefan Gehlen, and Christoph Von der Malsburg. Strategies and benefits of fusion of $2 \mathrm{~d}$ and $3 \mathrm{~d}$ face recognition. In Proceedings of the 2005 IEEE Computer Society Conference on Computer Vision and Pattern Recognition (CVPR'05) - Workshops - Volume 03, pages 174-, Washington, DC, USA, 2005. IEEE Computer Society. 
[27] ISO. ISO/IEC 14496-2:2001: Information technology - Coding of audio-visual objects - Part 2: Visual.

[28] Anil K. Jain, Arun Ross, and Salil Prabhakar. An introduction to biometric recognition. IEEE Trans. Circuits Syst. Video Techn, 14(1):4-20, 2004.

[29] Ioannis a Kakadiaris, Georgios Passalis, George Toderici, Mohammed N Murtuza, Yunliang Lu, Nikos Karampatziakis, and Theoharis Theoharis. Threedimensional face recognition in the presence of facial expressions: an annotated deformable model approach. IEEE transactions on pattern analysis and machine intelligence, 29(4):640-649, April 2007.

[30] Ioannis A. Kakadiaris, Georgios Passalis, George Toderici, Mohammed N. Murtuza, Yunliang Lu, Nikos Karampatziakis, and Theoharis Theoharis. Threedimensional face recognition in the presence of facial expressions: An annotated deformable model approach. IEEE Transactions on Pattern Analysis and $\mathrm{Ma}$ chine Intelligence, 29:640-649, 2007.

[31] Jan J. Koenderink. Solid Shape. Artificial Intelligence Series. MIT Press, Cambridge, MA, USA, 1990.

[32] Y Lee and D Marshall. Curvature based normalized 3D component facial image recognition using fuzzy integral. Applied Mathematics and Computation, 205(2):815-823, November 2008.

[33] David G. Lowe. Distinctive image features from scale-invariant keypoints. International Journal of Computer Vision, 60(2):91, November 2004.

[34] T. Maurer, D. Guigonis, I. Maslov, B. Pesenti, A. Tsaregorodtsev, D. West, and G. Medioni. Performance of geometrix activeID(TM) 3D face recognition engine on the FRGC data. In Workshop on Face Recognition Grand Challenge Experiments, pages III: 154-154, 2005.

[35] C. McCool, V. Chandran, S. Sridharan, and C. Fookes. 3D face verification using a free-parts approach. Pattern Recognition Letters, 29(9):1190-1196, July 2008.

[36] A. S. Mian, M. Bennamoun, and R. A. Owens. An efficient multimodal 2D-3D hybrid approach to automatic face recognition. IEEE Trans. Pattern Analysis and Machine Intelligence, 29(11):1927-1943, November 2007.

[37] G. Passalis, I.A. Kakadiaris, T. Theoharis, G. Toderici, and N. Murtuza. Evaluation of $3 \mathrm{~d}$ face recognition in the presence of facial expressions: an annotated deformable model approach. In Computer Vision and Pattern Recognition Workshops, 2005. CVPR Workshops. IEEE Computer Society Conference on, page 171, june 2005 .

[38] P. J. Phillips, P. J. Flynn, T. Scruggs, K. W. Bowyer, J. Chang, K. Hoffman, J. Marques, J. Min, and W. Worek. Overview of the face recognition grand challenge. In IEEE Computer Vision and Pattern Recognition or CVPR, pages I: $947-954,2005$.

[39] Chauã C Queirolo, Luciano Silva, Olga R P Bellon, and Maurício Pamplona Segundo. 3D face recognition using simulated annealing and the surface interpenetration measure. IEEE transactions on pattern analysis and machine intelligence, 32(2):206-219, February 2010. 
[40] R. Sala Llonch, E. Kokiopoulou, I. Tošić, and P. Frossard. 3D face recognition with sparse spherical representations. Pattern Recognition, 43(3):824-834, March 2010 .

[41] F. ter Haar and R. Veltkamp. 3D face model fitting for recognition, pages 652-664. Springer, 2008.

[42] Frank B. ter Haar and Remco C. Veltkamp. A 3D face matching framework for facial curves. Graphical Models, 71(2):77-91, March 2009.

[43] A. Vedaldi and B. Fulkerson. VLFeat: An open and portable library of computer vision algorithms. http://www.vlfeat.org/, 2008.

[44] Yingjie Wang and Chin-Seng Chua. Face recognition from 2d and 3d images using 3d gabor filters. Image and Vision Computing, 23(11):1018 - 1028, 2005.

[45] Yueming Wang, Jianzhuang Liu, and Xiaoou Tang. Robust 3D face recognition by local shape difference boosting. IEEE Trans. Pattern Anal. Mach. Intell, 32(10):1858-1870, 2010.

[46] Wikipedia. Principal curvature - Wikipedia, the free encyclopedia, 2011. [Online; accessed 07-February-2011].

[47] Chenghua Xu, Stan Li, Tieniu Tan, and Long Quan. Automatic 3D face recognition from depth and intensity Gabor features. Pattern Recognition, 42(9):18951905, September 2009.

[48] Guangpeng Zhang and Yunhong Wang. Robust 3D face recognition based on resolution invariant features. Pattern Recognition Letters, 32(7):1009-1019, May 2011.

[49] Y. Zhang and N.I. Badler. Face modeling and editing with statistical local feature control models. IJIST, 17(6):341-358, 2007. 


\section{APPENDIX A}

\section{GENERIC FACE MODEL}

points $=\left[\begin{array}{lllllllll}12.4935 & -74.738 & 80.435, & 19.5373 & -73.9317 & 75.031, & 69.0536 & 4.3135 & 22.2082, \\ 57.6345 & 16.0381 & 35.8274, & 10.2794 & 54.1794\end{array}\right.$ $50.5945,16.86361 .229149 .4818,27.367941 .428442 .483,21.7214 \quad 36.859646 .1105,22.29636 .999642 .4321,27.647141 .5342$ $45.9484,45.929924 .299444 .0513,9.1830535 .195951 .2195,13.818634 .526147 .5222,47.648934 .354936 .6268,18.619332 .3915$ $45.8887,18.461733 .496746 .2293,22.495532 .6375 \quad 42.3492,26.4709-27.8465 \quad 74.5094,26.151 \quad-28.2529 \quad 73.67,24.5422 \quad-27.524$ $75.68,26.7768-27.157274 .7614,23.8491-24.5338$ 78.5976, 27.5319 -27.7433 74.2552, $27.047-25.4775 \quad 75.3063,30.7666-27.5316$ 73.796, $26.4802-28.397974 .0574,20.9046-34.714685 .9085,25.1624-29.7573 \quad 76.4976,22.4832-18.966981 .318,16.9608-13.8023$ $84.4102,24.5173-27.833576 .1851,24.5422-27.524 \quad 75.68,43.453932 .08341 .1452,31.682731 .425946 .1149,83.1641-28.8041$ $-39.0521,75.2706-30.4749-14.7245,75.5327-23.4268-15.4032,75.5251-14.7051-18.557,49.9166 \quad 35.953 \quad 35.5241,66.2928-$ $48.7113-0.127351,21.495632 .426946 .3388,5.5465839 .9554 \quad 52.7312,18.4069-26.478982 .0324,17.3754-21.40784 .1501,60.21$

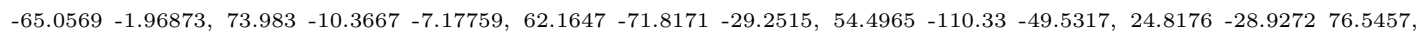
$26.5641-28.1453 \quad 74.4934,28.2369-27.908 \quad 74.0181, \quad 19.9252-30.9042 \quad 85.1598, \quad 28.7522 \quad-72.3565 \quad 63.1555, \quad 37.9987 \quad-70.6376-$

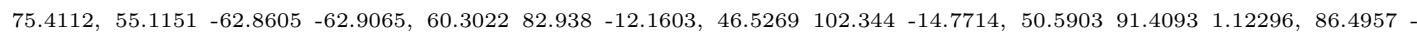
$12.917-54.5752,82.9086-12.3598-57.7517,83.2943-25.3556-48.3874,80.5532-26.5599-50.9019,78.6215-32.4024-45.9526$, $81.7799-31.614-42.2294,76.5914-35.4867-25.7088,81.3148-32.4274-26.822, \quad 71.5362-43.9539-24.5562,85.8378 \quad 8.88925-$

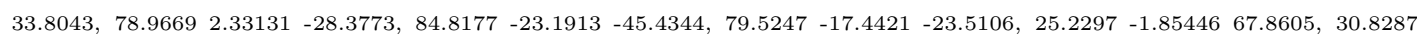
-3.07815 67.1204, $24.37426 .8943464 .7253,20.1103 \quad 0.52256 \quad 70.4654,22.20887 .4783468 .9758,52.9018 \quad 30.1433 \quad 30.7952,53.793$

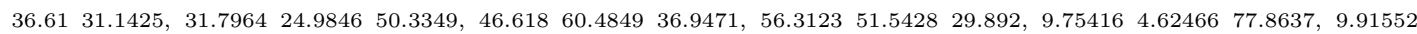

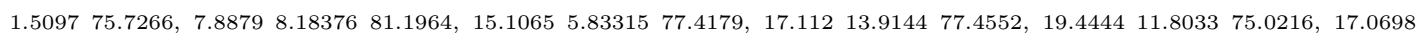
$15.00175 .3268,65.943179 .4172-35.2005,27.3304-30.266372 .73,27.0187-28.009672 .0068,-11.6825-75.168180 .5583,-18.7045$ -74.6595 75.1941, -8.41875 -82.9522 60.5519, -68.9372 4.99252 22.5598, -56.6493 17.3157 36.7043, $-10.397954 .658450 .4762,-$ $17.322261 .420949 .3868,-49.296935 .120535 .7823,-27.492541 .994242 .7495,-22.84737 .570942 .6094,-22.942338 .077145 .6915$, $-27.75242 .463245 .8531,-45.516725 .069544 .537,-9.0597735 .765351 .0832,-14.245135 .710747 .0745,-15.361132 .744148 .6181$ -47.6172 $34.94836 .6861,-19.991733 .401245 .0761,-20.349334 .74145 .0979,-22.910433 .147142 .4892,-25.8563-28.32875 .4628$,

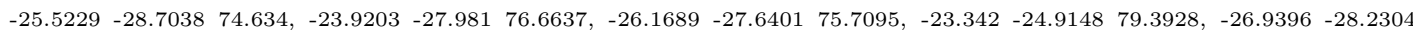

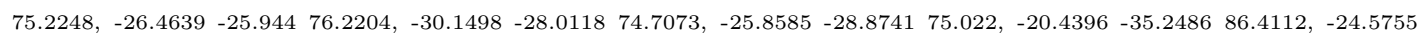
-30.2539 77.4758, -16.9196-14.0858 84.6942, -22.1262 -19.1954 81.9708, -23.9109 -28.2998 77.1656, -23.9242 -28.2497 76.6728,

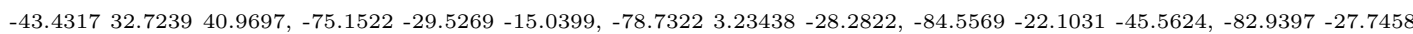
$-39.2363,-75.3745-22.5137-15.5944,-75.3121-13.83-18.6118,-66.4609-47.6607-1.01886,-5.6163840 .291152 .6123,-17.9317$ $-26.944582 .5895,-16.986-21.618884 .5236,-60.4638-64.0205-3.18327,-73.8747-9.60174-7.1316,-62.0551-71.1354-29.8349$,

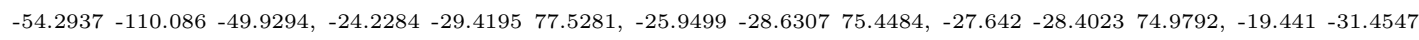
$85.6985,-28.5026-72.658863 .3171,-54.9327-62.3168-63.1019,-60.2738 \quad 82.9163-11.9851,-50.7867 \quad 91.108 \quad 1.28922,-86.2055$ $-11.7862-54.6371,-82.5855-11.2777-57.8025,-83.0351-24.2864-48.5249,-80.3372-25.531-51.0396,-78.4316-31.41-46.1414$ $-81.5673-30.5752-42.4292,-76.4519-34.5041-26.0335,-81.1649-31.3886-27.097,-71.4238-43.0178-25.0102,-85.5779 .90305$ $-33.6957,-79.3914-16.4788-23.6067,-25.2766-2.0995368 .0163,-24.18636 .4590165 .0626,-30.9381-3.0318167 .2084,-20.3014$

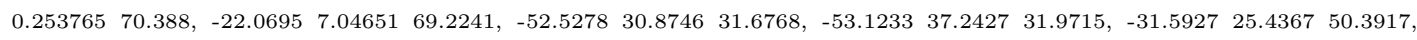

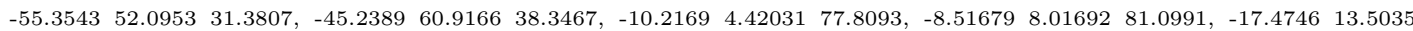
$77.4465,-17.356714 .563275 .4389,-19.561811 .4215 \quad 75.2134,-65.8122 \quad 79.7564 \quad-35.0616,-26.7309-30.6706 \quad 73.7236,-26.3992-$ $28.436772 .9607,-15.40185 .4678977 .4414,-10.25741 .3130175 .678,-22.530533 .320245 .9128,57.8177-102.651-9.9802,62.6251$

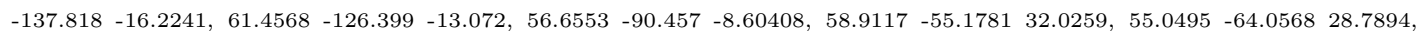
$\begin{array}{lllllllllllllll}68.1808 & 50.3315 & -1.09495, & 65.6038 & 37.2134 & 11.5756, & 75.6526 & 32.3172 & -42.4814, & 7.87641 & -117.565 & 33.1116, & 6.46626 & -95.8591\end{array}$ $34.1385,10.467-143.74127 .6995,11.9131-155.423 \quad 25.8965,0.101123-144.603 \quad 29.918,0.0827908-156.29128 .215,68.7015-$ $51.6488-26.6623,62.6904-48.1357-50.0904,56.053-125.35-51.4699,0.186481-118.6935 .435,62.0509-45.685532 .3067,63.6019$

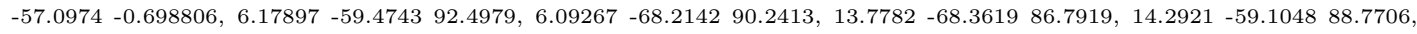
$21.5236-67.002480 .0914,22.4949-58.3865 \quad 81.0297,6.67026-51.0755 \quad 90.5579, \quad 0.395696-51.4988 \quad 91.683,0.263596 \quad-59.5767$ $93.5328,5.74301-74.972182 .7545,9.15865-82.7676 \quad 60.4755,18.503424 .3175$ 53.9468, $13.399127 .550955 .513,53.9618-13.7975$ $56.054,54.3428-3.6581654 .3312,41.42381 .7945262 .7738,42.6931-7.6258765 .1113,63.5592-8.124 \quad 40.4984,68.2307 \quad 19.1175$ 
$14.2049,-0.52205327 .591575 .0122,3.9140426 .8276 \quad 74.8285,22.47786 .5734 \quad 33.7926,32.200684 .0130 .2453,0.449615-86.6437$

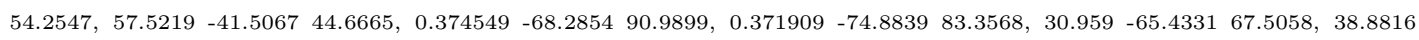
-69.971 51.5881, $40.5453-61.8735 \quad 57.274,0.530719-95.612 \quad 35.6019,4.31824 \quad 23.1592 \quad 82.318,10.1582 \quad 20.3858 \quad 79.0589,9.48361$ $23.534772 .3243,28.55217 .8199162 .6776,22.268512 .311163 .9054,30.3661$-31.8213 73.2939, $16.5584 \quad 3.2743975 .3584,15.6532$ $0.80730174 .4829,10.7086-1.37205 \quad 77.2108,15.8021-2.9365973 .991,15.2472-1.31737 \quad 73.7606,2.95087 \quad 6.3921481 .9683,-$ $0.4747445 .7124983 .5385,-0.520563 .3643581 .5399,2.738694 .1706580 .1481,20.7223-2.2898670 .7156,5.0573317 .322287 .7358$, $5.0799912 .865786 .9617,10.553712 .344582 .8095,10.895416 .246682 .3828,29.898-45.4852 \quad 74.6756,27.9265-37.165276 .6873$, $21.9116-41.250583 .0276,22.1998-48.86381 .1965,30.1715$-19.0921 74.9148, 25.9005 - 16.2783 77.3334, 3.12436 1.61263 79.7567, -0.518005 1.21274 80.8359, $20.045912 .261172 .1487,19.993412 .1308$ 72.9207, 20.7149 9.89377 72.8632, 19.76274 .8690772 .9856 , $0.026912-9.9177689 .1774,6.44597-8.3882987 .7988,5.34782-5.0202984 .3679,-0.0763932-5.8973384 .6771,6.96043-43.2645$ $88.9456,14.6858-42.684987 .2317,16.508215 .764174 .0749,0.787191-43.420689 .1519,59.209237 .723123 .7168,31.631273 .1294$ $38.6397,44.751567 .586832 .4608,45.701576 .8326 \quad 23.3984,57.1386 \quad 56.377524 .1811,58.932564 .849 \quad 13.8145,20.047453 .4311$ $47.0277,27.2878 \quad 56.034745 .2206,8.63416 \quad 31.3977 \quad 57.4463,3.26953 \quad 35.2408 \quad 58.8933,35.2979 \quad 18.0026 \quad 52.2362,23.15 \quad 21.7008$ 53.3397, $59.344124 .179924 .4947,35.3454 \quad 64.40242 .3557,44.256244 .034740 .4477,38.792346 .665 \quad 42.4923,32.02446 .7161$ $43.2257,24.589543 .50944 .0573,18.1116 \quad 37.6598 \quad 44.7995, \quad 50.9506 \quad 32.5596 \quad 34.1205,49.3873 \quad 34.461 \quad 35.462,49.9796 \quad 30.6604$ $35.1652,33.558144 .044844 .6069,43.33941 .313641 .8583,46.227138 .2257 \quad 39.0162,47.6073 \quad 34.952 \quad 36.7386,46.8969 \quad 34.7172$ $33.5698,46.902234 .20933 .6541,36.60454 .722942 .6416,49.282441 .2032 \quad 37.4921,45.160945 .904341 .2801,32.002127 .9414$ $50.4292,44.996927 .963244 .3338,21.5346 \quad 29.9378 \quad 48.323,17.9825 \quad 31.6707 \quad 47.1073,14.24246 .306 \quad 47.7548,10.4164 \quad 38.8557$ $48.9207,16.364133 .666246 .4139,15.42938 .370346 .3096,43.917731 .839644 .2019,31.866831 .280549 .3228,21.265134 .5348$ $41.5228,21.463633 .45941 .5882,45.498338 .003936 .2743,-0.17279255 .510751 .6939,-0.17047648 .30752 .3531,3.8333947 .8789$ $52.0876,6.0665855 .733851 .5593,15.357931 .853648 .8824,21.3775 \quad 27.317649 .6206,17.8743-26.474580 .3551,24.2619-27.9714$ $75.3035,7.01816-14.168389 .4502,0.0497649-14.486891 .3776,6.75455-17.754488 .9829,17.7938-17.8756 \quad 84.5756,4.87877$ $22.097588 .8113,18.4069-26.478982 .0324,18.6885-27.162582 .8377,14.43-30.372590 .5389,12.2552-25.173688 .0959,5.02037$

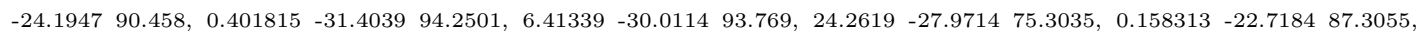

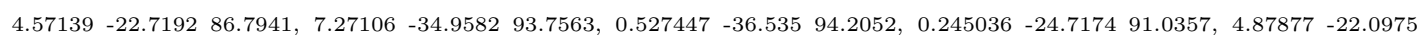

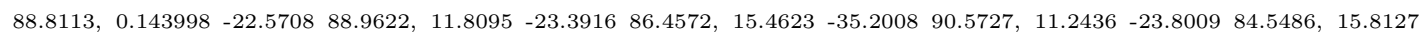

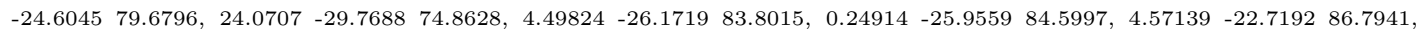
$6.31725-22.113382 .8474,11.2867-26.842581 .6981,26.165-20.961767 .5815,28.7247-23.91864 .3997,14.9514-16.608675 .6738$, $26.4292-39.172672 .665,23.7503-38.8427$ 76.3475, $5.83273-14.0378$ 78.0296, $11.4013-36.265283 .5064,4.66924-35.626685 .569$, $28.6195-39.196667 .884,76.0172-18.2166-54.3007,78.41537 .02202-49.0199,75.72418 .4147-30.7091,75.20243 .71589-19.0393$, $72.6121-30.6435-45.1339,78.3146-24.6957-20.3121,86.2776 \quad 14.5763-41.8238,83.0321 \quad 15.6593-44.9626,90.5292-0.328435$ $-52.774,83.057-1.26839-28.7518,77.1015-4.73497-56.5567,81.5916-14.7669-39.8331,15.4753 \quad 111.088 \quad 11.5829,-0.392342$ $\begin{array}{llllllllllllll}112.978 & 11.4383, & -0.291976 & 86.5496 & 38.3838, & 12.1707 & 87.2985 & 36.4749, & 21.8359 & 74.3377 & 42.4153, & 3.64057 & 8.39845 & 84.0808,\end{array}$

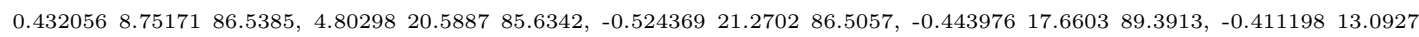
$89.7384,-0.63905623 .869282 .7736,-0.11650972 .378246 .1699,-0.11118365 .80349 .3309,11.662466 .832448 .4564,11.55372 .655$ $45.4397,-0.20900936 .250859 .0973,3.1671141 .694753 .5388,-0.11284142 .531453 .6973,0.158313-22.718487 .3055,0.166565$ -21.7284 82.8696, $0.143998-22.570888 .9622,0.0591444-17.74790 .6683,0.0122504-13.849877 .9662,27.3015 \quad 107.11611 .2132$, $25.103864 .5795 \quad 46.822,33.211343 .77741 .5498,30.461548 .8804 \quad 44.5433,21.978 \quad 45.319845 .3702,38.0111 \quad 101.2178 .19427$, $38.968443 .745143 .7373,38.386948 .803843 .8395,38.629743 .690240 .9668,45.911751 .542238 .9658,47.3675 \quad 40.063 \quad 37.5005$, $43.009341 .164438 .87,52.156144 .968334 .3181,75.4953 \quad 16.8608-67.504,71.6132-21.5642-72.1831,73.5838-1.94505-78.3852$, $\begin{array}{llllllllllllll}65.9676 & -38.1485 & -60.5334, & 26.4253 & -84.6483 & 39.7787, & 49.2801 & -64.4991 & 42.3895, & 22.7154 & 118.635 & -13.7692, & 51.0115 & -55.4309\end{array}$ $48.1554, \quad 20.7966-11.7658 \quad 80.3284, \quad 14.0531 \quad-8.35397 \quad 82.9556, \quad 33.3078-28.866 \quad 72.4602,34.0491 \quad-21.7115 \quad 72.3054,70.6245-$ $29.8733-0.915649,72.2784-21.4019-2.95162,51.464626 .1443 \quad 34.7576,15.461218 .268868 .5809,17.24689 .9012877 .9023,19.419$ $7.1168875 .1023,49.9661-33.801257 .6014,40.9393-26.757366 .9875,46.0598-43.3325$ 59.7794, $62.6263-18.645541 .687,39.2082$ -50.8583 64.1938, $31.1871-55.9507 \quad 70.7979,48.973815 .700646 .9528,38.89-34.438668 .6233,34.9782-40.294370 .8083,14.2453$ $-50.419786 .9841,10.679118 .442881 .1042,68.4949-11.789624 .3864,66.4451-23.03127 .3154,7.0656345 .624551 .0577,20.8316$ $26.362476 .2711,19.9546-18.7397$ 72.5804, $28.4102-139.864$ 19.2197, $48.9443-146.75 .76544,45.1443-134.3466 .99645,38.1331$

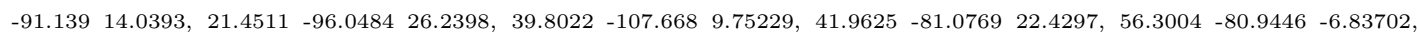

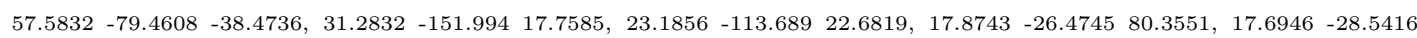

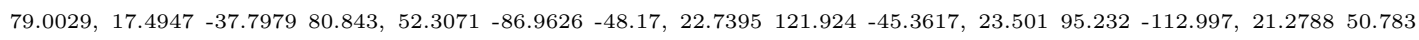
$-141.598,-0.114741126 .024-47.1434,20.12239 .54896-144.864,18.6225-28.4077-134.629,16.2811-56.3012-101.89,0.169821$

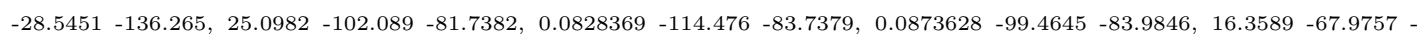
$92.0328,0.115493-66.7523-90.9145,0.0980315-85.922-85.1042,48.3548 \quad 103.997-42.4084,46.669745 .3723-126.271,47.7388$ $83.8455-99.807,44.50826 .68152-130.723,41.9613-27.7412-119.149,39.0485-55.0061-89.9712,25.5095-87.2959-79.8659$, $66.37162 .985-78.3406,65.896835 .7668-103.637,59.4023-25.8115-98.4553,63.23073 .34613-110.337,55.5652-50.5372-77.623$,

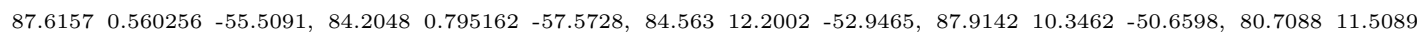

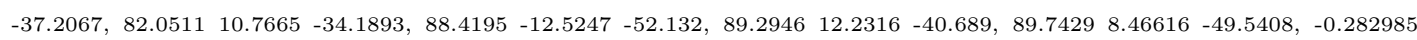
$123.421-14.4885,0.13372997 .5024-116.746,24.9765-115.763-79.8524,0.2285952 .1035-144.063,0.205904 \quad 10.9432-148.267$, $0.127768-56.0534-105.589,-57.7392-102.343-10.67,-61.2959-126.342-13.2573,-62.4594-137.818-16.224,-39.407-107.648$ $8.86602,-37.6083-91.031612 .5107,-56.6203-89.9497-9.52946,-56.1979-62.458426 .7344,-60.1096-53.3859 \quad 30.253,-65.3215$ $37.753512 .0483,-67.963950 .7218-0.686169,-75.1847 \quad 17.8323-67.4442,-75.3613 \quad 33.1346-42.3129,-5.66586-95.913433 .8855$,

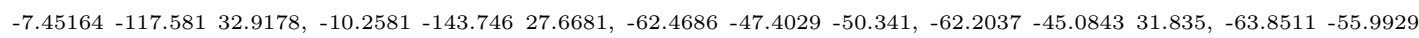
$-1.81805,-5.58995-59.596792 .6153,-13.3713-59.335388 .9355,-12.6892-68.918186 .9435,-5.36905-68.420790 .3715,-21.7469$ 


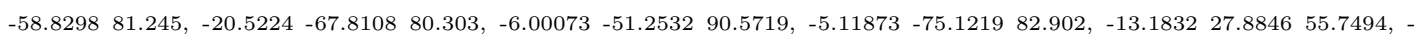
$\begin{array}{lllllllllllllll}18.3609 & 24.3963 & 54.1954, & -54.0038 & -13.2559 & 56.2434, & -42.7749 & -7.36421 & 65.2238, & -41.4168 & 2.01699 & 62.8871, & -53.0652 & -3.1007\end{array}$

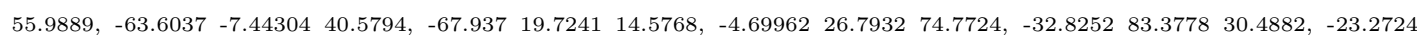
$86.036333 .9948,-57.7761-41.153244 .6965,-30.6549-65.7515 \quad 67.5808,-40.4106-62.055257 .188,-38.9263-70.024751 .6356$,

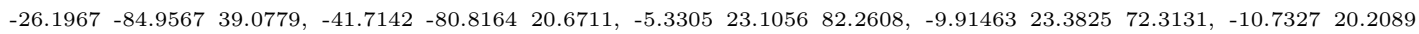

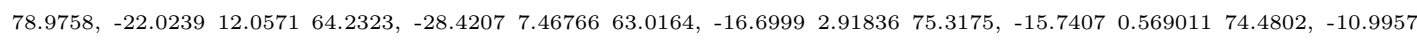
$-1.5223977 .2569,-15.3678-1.5525273 .771,-15.9133-3.20267 \quad 74.0251,-3.916536 .4090781 .9801,-3.798444 .1635980 .0154,-$ $20.9279-2.621870 .664,-5.8805417 .256987 .5852,-11.4927 \quad 16.038282 .5579,-11.121412 .089582 .8655,-5.8241612 .760586 .9629$,

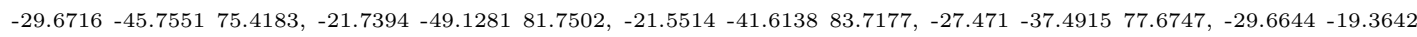
$75.639,-25.3899-16.59978 .039,-4.209461 .5907879 .5282,-20.077211 .8143 \quad 72.4372,-20.70959 .4391973 .1161,-20.061311 .694$ $73.2273,-19.83444 .55824 \quad 73.0024,-5.73582-5.4796284 .4893,-6.54135 \quad-8.5042987 .8572,-14.0427 \quad-42.9218 \quad 87.5046,-5.86407$

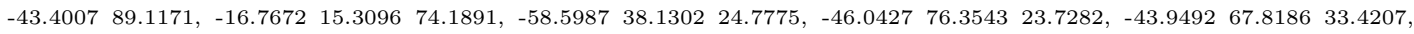

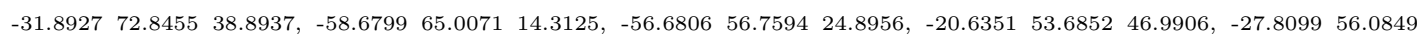
$45.3129,-8.7334131 .669457 .49,-3.6048535 .3272 \quad 58.8763,-22.830121 .9816 \quad 53.4765,-35.1797 \quad 18.432152 .3087,-58.996 \quad 24.829$ $25.1905,-34.714464 .449242 .9184,-44.065444 .616140 .8096,-38.651847 .187342 .9027,-31.973647 .295943 .4088,-24.743944 .252$ $44.1867,-19.340938 .822144 .227,-50.658833 .258134 .5185,-49.869231 .360635 .4329,-33.377444 .550644 .9506,-43.250241 .8217$ $41.9531,-46.108938 .682839 .305,-46.768534 .853534 .034,-46.761735 .361133 .9707,-47.571435 .531136 .8039,-35.898154 .7289$ $43.3759,-49.011641 .732538 .3989,-44.81946 .530341 .6434,-31.805228 .478150 .3892,-44.80928 .675444 .5131,-21.763430 .6206$ $48.2726,-18.589432 .562746 .6258,-10.378339 .636348 .7123,-14.327846 .531147 .6183,-17.464834 .79345 .5917,-16.112739 .5588$ $45.6567,-43.8843 \quad 32.531244 .1275,-31.8904 \quad 31.8542 \quad 49.1829,-31.773131 .967646 .0477,-21.7665 \quad 35.0146 \quad 41.7263,-21.9179$ $33.933841 .7819,-45.450138 .525136 .6191,-6.3598256 .0871 \quad 51.4407,-4.2407248 .4475 \quad 51.8871,-21.3599 \quad 27.652 \quad 49.6762,-$ $17.3711-26.732180 .8407,-23.6199-28.6671 \quad 76.3128,-6.95896-14.238989 .5792,-6.60082-17.943989 .1356,-17.5486-18.1024$ $84.8973,-4.49386-22.210288 .902,-18.2455-27.7591 \quad 83.4365,-17.9313-26.9446 \quad 82.5895,-13.6703 \quad-30.6045 \quad 90.8928,-4.48887$ $-24.201290 .5355,-11.7007-25.318288 .3402,-5.13416-30.426594 .0444,-23.6167-28.3993 \quad 76.3088,-4.16444-22.759786 .8686$,

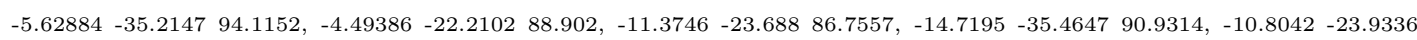
$84.765,-15.4072-24.8236 \quad 80.0722,-23.4005 \quad-30.1376 \quad 75.872,-4.00198-26.0158 \quad 83.8393,-4.16444 \quad-22.7597 \quad 86.8686,-5.96415$

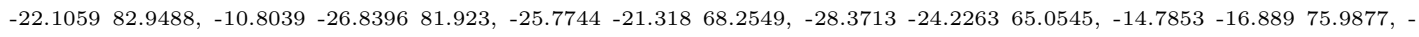
$26.0411-39.3857 \quad 73.7696,-23.2235-39.0365 \quad 77.1361,-3.67625-35.5186 \quad 85.6986,0.494278-35.712185 .6401,-5.80285-14.2028$ $78.1476,-10.638-36.2358 \quad 83.7496,-28.3483-39.438 \quad 68.9258,-75.7149-17.2465-54.3762,-74.9804 \quad 4.5139-18.8952,-75.4432$ $19.2279-30.5267,-72.436-29.7361-45.3063,-78.2119-23.7253-20.5112,-82.671916 .6589-44.8553,-85.9373 \quad 15.6147-41.7173,-$ $82.8259-0.288026-28.6926,-90.20140 .852684-52.7696,-76.7813-3.74687-56.5636,-78.13357 .98803-48.9541,-81.4077-13.7427$ -39.8995, - $-16.1949110 .827 \quad 11.6369,-12.9272 \quad 87.0047 \quad 36.6099,-22.5193 \quad 74.2174 \quad 42.4069,-4.4529 \quad 8.37948 \quad 83.9826,-5.68305$ $20.464985 .5916,-12.159472 .738145 .46,-12.141966 .86948 .4327,-3.3926141 .697453 .4671,-27.9142 \quad 106.71511 .3078,-26.3077$ $64.669346 .6408,-33.178144 .209741 .936,-30.577549 .337544 .7308,-22.297846 .088145 .6333,-38.4764100 .7898 .32094,-46.7334$ $102.145-14.6931,-38.892744 .218343 .9138,-37.96649 .307144 .1508,-38.731144 .167741 .2384,-45.09452 .152240 .0857,-47.1991$ $40.619538 .2615,-42.967841 .676739 .1555,-51.079845 .6195 \quad 35.7462,-71.3613-20.6339-72.2648,-73.2821-0.955448-78.3979$,

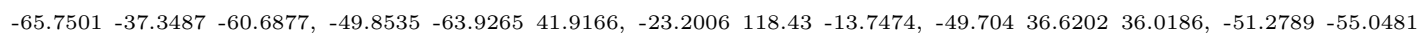
$47.9354,-20.4464-12.036780 .8201,-14.1382-8.5710383 .3025,-33.5378-21.9518 \quad 73.0867,-32.7321-29.2307 \quad 73.4748,-68.6593$ $-50.7612-27.1704,-70.6393-29.0493-1.26033,-72.2733-20.612-3.10354,-51.064126 .902535 .3344,-15.637417 .947968 .6744$,

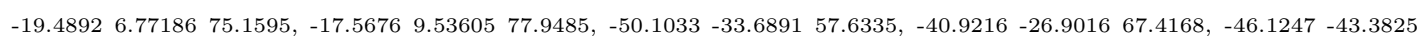
$60.04,-62.8104-18.008941 .5411,-30.8777-56.2742 \quad 71.1864,-39.3094-51.0464 \quad 64.6484,-48.2394 \quad 16.6952 \quad 47.3725,-38.9043$

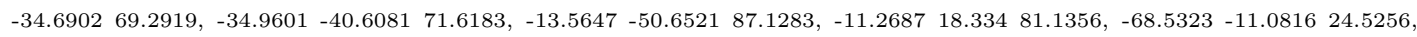

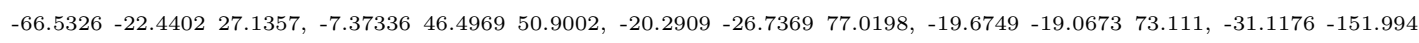

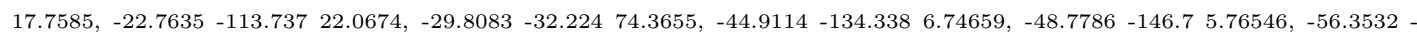
$80.2496-7.92382,-57.4073-78.9595-38.8941,-28.1618-139.878 \quad 19.0738,-20.9894-96.1488 \quad 25.052,-17.3706-26.732180 .8408$,

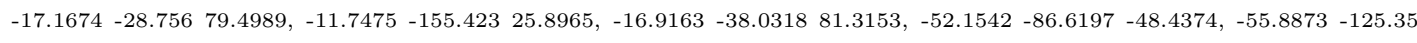
$-51.4698,-22.9173121 .913-45.3557,-21.007751 .0912-141.533,-23.219695 .4898-112.965,-19.70289 .8506-144.866,-18.2014$ $-28.1553-134.658,-16.0158-56.1377-101.924,-25.3199-87.1748-79.9176,-24.8108-115.763-79.8524,-16.1219-67.8428-92.068$, -48.3974 104.109-42.3612, -46.2712 $46.029-126.228,-47.459 \quad 84.3797-99.7489,-44.1095 \quad 7.33599-130.734,-41.6246-27.1806-$ $119.21,-38.8092-54.6003-90.0558,-37.7854-70.331-75.5172,-24.9236-102.043-81.7575,-65.5335 \quad 36.6723-103.591,-66.0999$ $63.7342-78.2519,-62.87534 .24807-110.357,-59.107-25.0281-98.5306,-55.3499-49.9185-77.7552,-87.2677 \quad 1.70134-55.4997$,

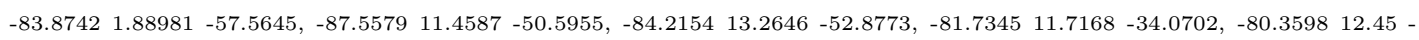
$37.0871,-88.127-11.3682-52.1915,-89.033313 .3215-40.5912,-89.39549 .60738-49.4861]$;

triangles $=[0,212,1,-1,17,18,25,-1,22,20,17,-1,30,17,31,-1,35,66,64,-1,375,69,494,-1,70,375,373,-1,375,372$, $496,-1,42,19,43,-1,46,44,454,-1,27,49,48,-1,55,56,57,-1,369,36,35,-1,59,61,364,-1,35,196,66,-1,71,72,73,-1$, $74,73,75,-1,83,81,84,-1,293,294,292,-1,85,86,87,-1,89,363,358,-1,90,355,356,-1,91,92,93,-1,112,111,119,-1$, $116,111,114,-1,124,125,111,-1,159,672,665,-1,672,668,158,-1,672,772,129,-1,669,672,774,-1,135,136,113,-1$, $139,734,137,-1,147,148,687,-1,152,150,661,-1,160,161,162,-1,163,164,161,-1,172,173,174,-1,176,654,660,-1$, $177,652,651,-1,171,178,170,-1,0,211,212,-1,22,17,49,-1,368,197,416,-1,34,69,375,-1,489,59,364,-1,494,372$, $375,-1,369,375,70,-1,380,391,390,-1,56,405,57,-1,376,400,419,-1,201,196,39,-1,238,423,24,-1,418,453,186,-1$, $52,417,230,-1,455,461,54,-1,68,493,492,-1,492,371,365,-1,365,68,492,-1,495,375,496,-1,473,53,482,-1,293$, $292,38,-1,91,93,528,-1,116,142,111,-1,518,664,697,-1,672,129,130,-1,661,150,767,-1,668,672,159,-1,672,669$, $772,-1,679,678,675,-1,687,148,686,-1,673,699,681,-1,706,520,133,-1,731,118,705,-1,545,698,509,-1,543,544$, 
$145,-1,742,735,146,-1,771,770,128,-1,666,771,671,-1,671,771,128,-1,127,155,157,-1,127,131,665,-1,752,750$, $759,-1,98,700,598,-1,142,121,141,-1,706,127,157,-1,373,375,67,-1,67,375,495,-1,672,773,774,-1,672,158,773$, $-1,599,98,598,-1]+1$;

quads $=[2,3,219,442,-1,4,5,390,320,-1,7,8,6,9,-1,11,41,281,214,-1,321,12,309,11,-1,294,13,312,305,-1,14$, $315,314,15,-1,19,17,20,21,-1,24,23,50,238,-1,25,18,90,89,-1,28,29,328,21,-1,1,212,417,52,-1,302,410,293$, $38,-1,77,412,302,38,-1,40,313,33,16,-1,7,15,314,8,-1,310,291,311,12,-1,68,367,45,37,-1,183,47,461,181,-1$, $48,49,17,30,-1,48,51,26,27,-1,53,54,461,482,-1,61,60,63,62,-1,61,59,58,60,-1,64,63,34,65,-1,64,66,62,63$, $-1,67,493,68,373,-1,69,60,58,494,-1,275,79,80,277,-1,82,81,244,247,-1,16,315,14,40,-1,95,94,724,535,-1,97$, $96,623,679,-1,99,100,101,102,-1,134,104,529,587,-1,105,106,104,613,-1,610,617,107,599,-1,620,621,108,109$, $-1,180,110,619,618,-1,114,111,113,115,-1,117,118,731,143,-1,112,119,176,177,-1,122,123,115,630,-1,127,706$, $133,707,-1,665,672,130,156,-1,145,544,93,92,-1,700,98,692,607,-1,607,694,166,700,-1,109,101,100,620,-1,597$, $615,105,616,-1,132,138,662,128,-1,503,742,140,504,-1,142,141,124,111,-1,116,143,121,142,-1,144,141,121,120$, $-1,146,759,750,742,-1,151,152,153,154,-1,132,159,665,131,-1,152,151,149,150,-1,671,128,662,663,-1,156,130$, $154,155,-1,153,157,155,154,-1,128,770,158,668,-1,584,168,169,581,-1,170,179,557,556,-1,171,170,556,676,-1$, $107,605,98,599,-1,180,108,621,110,-1,81,83,381,244,-1,200,39,425,443,-1,183,181,452,449,-1,448,182,183$, $449,-1,450,452,181,184,-1,184,181,461,455,-1,185,186,44,201,-1,187,188,366,189,-1,189,413,484,483,-1,193$, $192,194,195,-1,196,46,197,368,-1,182,198,47,183,-1,192,190,199,194,-1,202,203,204,205,-1,204,206,207,205$, $-1,202,208,209,210,-1,211,0,204,203,-1,213,214,428,237,-1,215,216,217,218,-1,216,219,3,437,-1,3,2,220,285$, $-1,215,218,432,431,-1,221,222,282,392,-1,223,224,405,400,-1,225,212,211,228,-1,226,200,443,434,-1,211,203$, $227,228,-1,229,52,230,231,-1,417,453,418,230,-1,191,212,225,232,-1,233,234,235,222,-1,73,72,236,237,-1,239$, $84,81,240,-1,241,242,243,82,-1,244,245,246,247,-1,248,74,243,242,-1,249,250,251,252,-1,253,254,255,256,-1$, $257,258,23,24,-1,259,260,268,267,-1,222,235,281,282,-1,261,262,263,75,-1,82,247,259,241,-1,262,87,86,263$, $-1,75,263,264,74,-1,265,266,267,268,-1,71,248,258,257,-1,269,270,346,340,-1,23,28,21,20,-1,87,262,261,271$, $-1,270,255,26,346,-1,272,269,340,341,-1,80,273,188,277,-1,224,274,275,276,-1,275,277,278,276,-1,286,79,275$, $274,-1,279,280,401,5,-1,282,281,41,393,-1,283,284,236,217,-1,3,285,76,427,-1,79,286,301,409,-1,80,79,409$, $412,-1,77,76,285,273,-1,287,288,406,296,-1,288,289,295,406,-1,290,9,295,289,-1,290,291,7,9,-1,310,15,7$, $291,-1,311,291,290,404,-1,403,289,288,407,-1,13,298,299,300,-1,280,301,286,401,-1,303,302,412,409,-1,10$, $305,304,78,-1,78,304,306,322,-1,304,305,312,313,-1,307,306,40,14,-1,308,309,12,311,-1,290,289,403,404,-1$, $313,312,32,33,-1,32,312,13,300,-1,410,297,298,293,-1,317,318,319,320,-1,213,284,322,321,-1,214,213,321,11$, $-1,41,11,309,444,-1,321,322,306,307,-1,323,324,19,42,-1,324,18,17,19,-1,326,325,266,265,-1,325,327,43,328$, $-1,325,328,29,266,-1,328,43,19,21,-1,43,327,329,42,-1,30,31,330,331,-1,51,48,30,331,-1,332,51,331,333,-1$, $332,333,334,336,-1,335,336,334,342,-1,337,25,89,349,-1,338,339,350,351,-1,336,335,341,340,-1,342,334,343$, $344,-1,333,345,343,334,-1,344,343,339,338,-1,331,330,345,333,-1,346,26,51,332,-1,336,340,346,332,-1,323,42$, $329,352,-1,22,50,23,20,-1,339,347,354,350,-1,339,343,345,347,-1,348,323,352,353,-1,360,357,348,353,-1,349$, $89,358,359,-1,351,350,362,657,-1,361,362,350,354,-1,356,363,89,90,-1,365,366,367,68,-1,366,365,413,189,-1$, $368,62,66,196,-1,63,60,69,34,-1,35,36,426,425,-1,370,371,492,493,-1,365,371,490,374,-1,70,373,68,37,-1,36$, $37,45,426,-1,369,70,37,36,-1,376,377,378,379,-1,380,274,224,223,-1,381,382,245,244,-1,249,383,384,385,-1$, $249,385,386,250,-1,259,247,246,260,-1,381,250,386,382,-1,384,383,233,387,-1,388,389,390,391,-1,392,282,393$, $394,-1,395,396,353,352,-1,397,395,352,329,-1,398,327,325,326,-1,398,397,329,327,-1,399,360,353,396,-1,223$, $400,376,379,-1,402,295,9,6,-1,404,403,280,279,-1,286,274,380,401,-1,301,280,403,407,-1,57,405,224,276,-1$, $303,407,288,287,-1,402,408,406,295,-1,303,409,301,407,-1,55,57,276,278,-1,410,287,296,297,-1,302,303,287$, $410,-1,316,297,296,411,-1,308,279,5,4,-1,308,311,404,279,-1,317,320,390,389,-1,201,44,46,196,-1,364,414$, $415,374,-1,379,378,388,391,-1,379,391,380,223,-1,88,55,278,187,-1,278,277,188,187,-1,316,299,298,297,-1,77$, $273,80,412,-1,191,232,199,190,-1,186,185,420,418,-1,267,266,29,422,-1,258,421,28,23,-1,24,423,424,257,-1$, $188,273,285,220,-1,77,38,292,76,-1,413,365,374,415,-1,414,364,368,416,-1,220,2,45,367,-1,76,292,294,427$, $-1,425,426,442,443,-1,213,237,236,284,-1,73,237,261,75,-1,86,85,429,430,-1,263,86,430,264,-1,218,72,424$, $432,-1,72,218,217,236,-1,259,267,422,241,-1,215,431,226,434,-1,420,226,431,433,-1,219,216,215,434,-1,1,52$, $229,206,-1,229,231,435,436,-1,305,10,427,294,-1,437,3,427,10,-1,227,203,202,210,-1,438,433,431,432,-1,435$, $439,253,436,-1,216,437,283,217,-1,440,208,202,205,-1,440,205,207,256,-1,283,437,10,78,-1,281,235,428,214$, $-1,87,271,234,441,-1,200,226,420,185,-1,443,442,219,434,-1,1,206,204,0,-1,269,208,440,270,-1,255,270,440$, $256,-1,304,313,40,306,-1,322,284,283,78,-1,269,272,209,208,-1,41,444,319,393,-1,310,307,14,15,-1,310,12$, $321,307,-1,394,393,319,318,-1,441,383,249,252,-1,74,264,240,243,-1,441,234,233,383,-1,4,320,319,444,-1,308$, $4,444,309,-1,258,248,242,421,-1,445,90,18,324,-1,355,90,445,446,-1,421,242,241,422,-1,348,445,324,323,-1$, $446,445,348,357,-1,237,428,271,261,-1,221,387,233,222,-1,271,428,235,234,-1,251,250,381,83,-1,429,251,83$ $84,-1,430,429,84,239,-1,264,430,239,240,-1,87,441,252,85,-1,85,252,251,429,-1,447,456,448,449,-1,447,449$, $452,457,-1,450,453,417,451,-1,450,451,457,452,-1,435,433,438,439,-1,435,231,420,433,-1,230,418,420,231,-1$, $438,423,238,439,-1,188,220,367,366,-1,39,200,185,201,-1,2,442,426,45,-1,450,184,454,453,-1,454,44,186,453$, $-1,46,454,184,455,-1,35,425,39,196,-1,238,254,253,439,-1,254,238,50,27,-1,253,256,207,436,-1,229,436,207$, $206,-1,330,31,337,458,-1,337,349,459,458,-1,459,349,359,460,-1,451,191,190,457,-1,456,447,192,193,-1,192$, $447,457,190,-1,347,345,330,458,-1,354,347,458,459,-1,361,354,459,460,-1,483,88,187,189,-1,465,462,463,498$, $-1,470,499,471,472,-1,474,473,482,475,-1,411,296,406,408,-1,479,477,484,486,-1,479,486,485,480,-1,480,485$, $487,481,-1,53,481,487,54,-1,56,55,88,476,-1,485,486,415,414,-1,486,484,413,415,-1,54,487,416,197,-1,485$, $414,416,487,-1,58,59,489,488,-1,489,490,491,488,-1,491,490,371,370,-1,61,62,368,364,-1,490,489,364,374,-1$, 
$372,494,58,488,-1,370,493,67,495,-1,491,496,372,488,-1,496,491,370,495,-1,369,65,34,375,-1,405,56,419,400$, $-1,376,419,497,377,-1,419,56,476,462,-1,497,419,462,465,-1,463,462,476,478,-1,478,476,88,483,-1,461,47,470$, $482,-1,470,47,198,499,-1,463,464,500,498,-1,464,466,501,500,-1,501,466,467,469,-1,469,467,468,502,-1,468$, $473,474,502,-1,482,470,472,475,-1,478,477,464,463,-1,477,479,466,464,-1,466,479,480,467,-1,467,480,481,468$, $-1,481,53,473,468,-1,483,484,477,478,-1,451,417,212,191,-1,26,255,254,27,-1,28,421,422,29,-1,438,432,424$, $423,-1,424,72,71,257,-1,240,81,82,243,-1,197,46,455,54,-1,71,73,74,248,-1,17,25,337,31,-1,133,519,725,707$, $-1,506,503,504,732,-1,504,505,733,732,-1,506,507,508,503,-1,503,508,735,742,-1,509,510,520,137,-1,511,512$, $514,663,-1,513,514,762,761,-1,740,195,194,517,-1,518,139,706,664,-1,140,743,505,504,-1,194,199,516,517,-1$, $521,522,523,524,-1,523,522,525,526,-1,521,210,209,527,-1,528,524,523,91,-1,529,530,549,710,-1,531,532,533$, $534,-1,95,535,534,719,-1,536,94,95,591,-1,532,531,713,714,-1,537,221,392,588,-1,538,539,681,686,-1,93,225$, $228,528,-1,519,540,716,725,-1,528,228,227,524,-1,541,542,543,145,-1,545,544,543,698,-1,515,232,225,93,-1$, $546,537,547,548,-1,161,549,550,162,-1,551,552,170,178,-1,553,179,554,555,-1,556,557,246,245,-1,558,555,554$, $163,-1,559,560,561,562,-1,563,564,565,566,-1,567,118,117,568,-1,268,260,569,574,-1,547,537,588,587,-1,570$, $164,571,572,-1,569,557,179,553,-1,572,571,174,173,-1,164,163,573,571,-1,265,268,574,575,-1,558,160,567,568$, $-1,576,577,640,643,-1,123,117,114,115,-1,173,578,570,572,-1,565,576,643,120,-1,577,272,341,640,-1,579,168$, $584,511,-1,538,580,581,582,-1,581,580,583,584,-1,169,592,582,581,-1,586,585,97,682,-1,134,587,588,680,-1$, $589,590,533,550,-1,591,95,709,165,-1,592,169,691,606,-1,169,168,694,691,-1,591,165,166,579,-1,594,593,601$, $688,-1,595,594,688,600,-1,595,600,102,596,-1,597,596,102,101,-1,685,596,597,616,-1,689,594,595,684,-1,107$, $603,604,605,-1,592,606,586,682,-1,607,608,691,694,-1,167,609,610,103,-1,611,609,167,625,-1,610,609,618,617$, $-1,611,612,108,180,-1,105,613,614,616,-1,595,596,685,684,-1,618,619,126,617,-1,107,617,126,603,-1,98,605$, $602,692,-1,317,623,624,318,-1,589,530,106,625,-1,530,529,104,106,-1,613,104,134,726,-1,612,611,625,106,-1$, $626,135,113,627,-1,627,113,111,112,-1,628,326,265,575,-1,629,628,630,136,-1,122,630,628,575,-1,630,115,113$, $136,-1,631,629,136,135,-1,124,632,633,125,-1,141,144,632,124,-1,144,634,636,632,-1,635,636,634,637,-1,635$, $637,335,342,-1,119,638,646,176,-1,639,338,351,647,-1,335,637,640,341,-1,342,344,641,635,-1,636,635,641,642$, $-1,344,338,639,641,-1,632,636,642,633,-1,144,120,643,634,-1,643,640,637,634,-1,631,135,626,648,-1,117,143$, $116,114,-1,644,639,647,650,-1,639,644,642,641,-1,626,645,649,648,-1,649,645,653,658,-1,176,646,655,654,-1$, $647,351,657,656,-1,647,656,659,650,-1,176,660,652,177,-1,671,663,514,513,-1,129,151,154,130,-1,131,127,707$, $708,-1,666,667,770,771,-1,769,666,671,670,-1,132,128,668,159,-1,132,131,708,138,-1,664,706,157,153,-1,378$, $377,673,674,-1,675,539,538,582,-1,676,556,245,382,-1,559,385,384,677,-1,559,562,386,385,-1,569,260,246,557$, $-1,676,382,386,562,-1,384,387,546,677,-1,388,678,679,389,-1,588,392,394,680,-1,395,648,649,396,-1,397,631$, $648,395,-1,398,326,628,629,-1,398,629,631,397,-1,649,658,399,396,-1,674,673,681,539,-1,683,99,102,600,-1$, $684,685,585,586,-1,682,675,582,592,-1,684,586,606,689,-1,538,686,148,580,-1,689,608,593,594,-1,683,600,688$, $690,-1,689,606,691,608,-1,580,148,147,583,-1,601,593,692,602,-1,608,607,692,593,-1,622,693,601,602,-1,97$, $585,614,96,-1,685,616,614,585,-1,317,389,679,623,-1,139,137,520,706,-1,696,695,661,670,-1,674,678,388,378$, $-1,674,539,675,678,-1,583,147,175,512,-1,583,512,511,584,-1,622,602,605,604,-1,694,168,579,166,-1,515,516$, $199,232,-1,509,698,701,510,-1,122,575,574,703,-1,568,117,123,702,-1,569,553,703,574,-1,118,567,704,705,-1$, $536,591,579,511,-1,598,700,166,165,-1,696,670,671,513,-1,697,664,661,695,-1,94,536,662,138,-1,599,598,165$, $709,-1,708,707,725,724,-1,589,550,549,530,-1,161,164,570,549,-1,174,711,712,172,-1,571,573,711,174,-1,162$, $532,714,704,-1,162,550,533,532,-1,540,713,531,716,-1,701,715,713,540,-1,535,716,531,534,-1,92,526,541,145$, $-1,541,717,718,542,-1,599,709,103,610,-1,709,95,719,103,-1,227,210,521,524,-1,720,714,713,715,-1,718,717$, $563,721,-1,533,590,719,534,-1,722,522,521,527,-1,722,564,525,522,-1,719,590,167,103,-1,529,710,547,587,-1$, $173,723,548,578,-1,519,510,701,540,-1,725,716,535,724,-1,92,91,523,526,-1,577,576,722,527,-1,565,564,722$, $576,-1,611,180,618,609,-1,167,590,589,625,-1,577,527,209,272,-1,134,680,624,726,-1,108,612,615,109,-1,106$, $105,615,612,-1,394,318,624,680,-1,723,560,559,677,-1,163,554,552,573,-1,723,677,546,548,-1,96,726,624,623$, $-1,109,615,597,101,-1,613,726,96,614,-1,555,558,568,702,-1,727,627,112,177,-1,727,177,651,728,-1,703,553$, $555,702,-1,645,626,627,727,-1,653,645,727,728,-1,549,570,578,710,-1,221,537,546,387,-1,578,548,547,710,-1$, $561,171,676,562,-1,712,178,171,561,-1,711,551,178,712,-1,573,552,551,711,-1,173,172,560,723,-1,172,712,561$, $560,-1,736,732,733,729,-1,732,736,730,506,-1,507,737,544,545,-1,507,506,730,737,-1,718,721,720,715,-1,718$, $715,701,542,-1,543,542,701,698,-1,720,721,731,705,-1,536,511,663,662,-1,520,510,519,133,-1,708,724,94,138$, $-1,734,508,507,545,-1,509,137,734,545,-1,508,734,139,735,-1,731,721,563,566,-1,731,566,121,143,-1,563,717$, $525,564,-1,541,526,525,717,-1,633,738,638,125,-1,739,646,638,738,-1,655,646,739,741,-1,737,730,516,515,-1$, $729,740,517,736,-1,736,517,516,730,-1,644,738,633,642,-1,739,738,644,650,-1,739,650,659,741,-1,514,512,175$, $762,-1,746,744,465,498,-1,474,475,750,752,-1,693,690,688,601,-1,756,763,761,754,-1,756,757,764,763,-1,757$, $758,765,764,-1,759,146,765,758,-1,687,753,175,147,-1,763,764,695,696,-1,513,761,763,696,-1,765,146,518,697$, $-1,765,697,695,764,-1,150,149,766,767,-1,767,766,768,769,-1,667,666,769,768,-1,153,152,661,664,-1,767,769$, $670,661,-1,772,149,151,129,-1,766,149,772,669,-1,158,770,667,773,-1,768,766,669,774,-1,773,667,768,774,-1$, $686,681,699,687,-1,673,377,497,699,-1,699,744,753,687,-1,497,465,744,699,-1,755,753,744,746,-1,762,175,753$, $755,-1,742,750,760,140,-1,760,751,743,140,-1,746,498,500,745,-1,745,500,501,747,-1,501,469,748,747,-1,469$, $502,749,748,-1,749,502,474,752,-1,750,475,472,760,-1,760,472,471,751,-1,755,746,745,754,-1,754,745,747,756$, $-1,747,748,757,756,-1,748,749,758,757,-1,758,749,752,759,-1,762,755,754,761,-1,737,515,93,544,-1,121,566$, $565,120,-1,123,122,703,702,-1,720,705,704,714,-1,567,160,162,704,-1,552,554,179,170,-1,146,735,139,518,-1$, $160,558,163,161,-1,111,125,638,119,-1,27,50,22,49,-1,127,665,156,155,-1,369,35,64,65,-1,380,390,5,401,-1$, $679,675,682,97,-1,293,298,13,294,-1]+1$ 


\section{VITA}

Tolga İnan was born in Ankara, Turkey in 1978. He received his B.S. and M.S. degrees both in Electrical Engineering from Middle East Technical University (METU), Ankara, Turkey in 2000 and 2003 respectively. He was a research and teaching assistant in the Department of Electrical and Electronics Engineering at METU between 2000 and 2007. He currently works as a senior researcher at Tübitak-UZAY (Space Technologies Research Institute of Scientific and Technological Research Council). His research interests include 3D face recognition, biometrics, pattern recogntion, machine learning and power quality measurement technologies.

His publications are as follows:

\section{Journal Papers}

- 3D Face Recognition with Local Shape Descriptors; Inan,T.;Halici,U.

IEEE Transactions on Information Forensics and Security Under Review

- Nationwide Real-Time Monitoring System for Electrical Quantities and Power Quality of the Electricity Transmission System; Demirci,T.;Kalaycioglu,A.;Kucuk,D.; et.al.

IET Generation, Transmission and Distribution, Volume 5, Issue 5, May 2011, Pages 540-550

- PQONT: A domain ontology for electrical power quality ;

Kucuk,D.;Salor,O.;Inan,T.; Advanced Engineering Informatics;

Volume 24, Issue 1, January 2010, Pages 84-95

- An extensible database architecture for nationwide power quality monitoring, Kucuk, D.; Inan, T. Salor, O. et.al.;

International Journal of Electrical Power and Energy Systems;

Volume 32, Issue 6, July 2010, Pages 559-570

- Mobile monitoring system to take nationwide PQ measurements on electricity transmission systems ; Salor,O.;Buhan,S.;Unsar,O; et.al. Measurement;Volume 42, Issue 4, May 2009, Pages 501-515 


\section{International Conference Papers}

- Building an Ontology for Flexible Power Quality Querying, Kucuk, D.; Salor, O.; Inan, T.; Cadirci, I.; ISCIS,2008

- PQStream: A Data Stream Architecture for Electrical Power Quality; D.Küçük,T.Ínan,B.Boyrazoğlu et.al;

International Workshop on Knowledge Discovery from Ubiquitous Data Streams; European Conference on Machine Learning;ECML 2007

- Electrical Power Quality of Iron and Steel Industry in Turkey; Ö. Salor,B.Gültekin,S. Buhan et.al;

IEEE Industry Applications Society Annual Meeting;IAS 2007

\section{National Conference Papers}

- Demirci, T., Kalaycığlu, A., Salor, Ö., Pakhuylu, S., İnan, T., Küçük, D., Güder, M., Can, T., Akkaya, Y., Bilgen, S., Çadırcı, I., Ermiş, M. 2008. Türkiye Elektrik İletim Sistemi için Yurt Çapında Güç Kalitesi İzleme A $\breve{g}$ ve Veri Değerlendirme Merkezi: Güncel Gelişmeler,

IEEE 16. Sinyal İşleme, İletişim ve Uygulamaları Kurultayı. Didim, Türkiye,2008

- Effects of 3D Registration on Subspace Based Face Recognition Methods ; Bülent Üstün, Uğur Halıcı, İlkay Ulusoy, Tolga İnan; IEEE 16. Sinyal İşleme, İletişim ve Uygulamaları Kurultayı. Didim, Türkiye,2008

- Probabilistic Face Pose Estimation; Tolga Ínan, Ílkay Ulusoy, Uğur Halıcı; Turkish Symposium on Artificial Intelligence and Neural Networks;TAINN 2006

\section{M.S. Thesis}

- T. İnan, "Four Quadrant Computer Based Motor Test System Development," Middle East Technical University, Ankara, Turkey, August. 2003. 